OFFICE OF CIVILIAN RADIOACTIVE WASTE MANAGEMENT

1. QA: QA

SPECIAL INSTRUCTION SHEET

Page: 1 of: 1

Complete Only Applicable Items

This is a placeholder page for records that cannot be scanned or microfilmed

2. Record Date

$04 / 18 / 2000$

3. Accession Number

4. Author Name(s)

MOL. 20000424.0694

N/A

5. Author Organization

N/A

6. Title

DATA QUALIFICATION REPORT: BOREHOLE STRATIGRAPHIC CONTACTS

7. Document Number(s)

TDR-NBS-GS-000007

8. Version

REV. 01

\begin{tabular}{l|l}
\hline 9. Document Type & 10. Medium \\
REPORT & OPTIC/PAPER
\end{tabular}

11. Access Control Code

PUB

12. Traceability Designator

DC $\# 25213$

13. Comments

THIS IS A ONE-OF-A-KIND DOCUMENT DUE TO THE COLOR GRAPHS ENCLOSED, SEE THE RPC FOR MORE

DETAILS

THIS ONE OF A KIND COLOR

DOCUMENT CAN BE LOCATED

THROUGH THE RECORDS

PROCESSING CENTER 
WBS: 1.2 .21 .3 .3

QA: Q/A

\section{Civilian Radioactive Waste Management System Management \&Operating Contractor}

Data Qualification Report:

Borehole Stratigraphic Contacts

Revision 01

TDR-NBS-GS-000007

March 2000

Prepared for:

U.S. Department of Energy

Yucca Mountain Site Characterization Office

P.O. Box 30307

North Las Vegas, Nevada 89036-0307

Prepared by:

TRW Environmental Safety Systems Inc.

1211 Town Center Drive

Las Vegas, Nevada 89144

Under Contract Number

DE-AC08-91RW00134 


\title{
Data Qualification Report: Borehole Stratigraphic Contacts
}

\author{
Revision 01 \\ Qualification Team: \\ Robert W. Clayton, Ph.D. \\ CRWMS M\&O/NEPO \\ URS \\ Clinton Lum, Ph.D. \\ CRWMS M\&O/NEPO \\ SNL
}

TABLE OF CONTENTS

Data Qualification Report..

Attachment I: Data and Transmittal Letter from TDMS

Attachment II: Query Letter and Response from Data Users

Attachment III: Cross Section from GFM3.1 Showing Logs

Attachment IV: Thickness Maps

Attachment V: Typical Geophysical Log Signatures for the Sample Contacts

Attachment VI: Contacts Examination Checklist

Attachment VII: Data Recommended to be Qualified

Attachment VIII: Special Case Data Recommended to be Qualified

NOTE: The row and column headings on the spreadsheet Attachments (I, VI, VII, and VIII) are for cell referencing only, and do not correlate between Attachments. To correlate between Attachments, use borehole identifiers.

NOTE TO THIS REVISION: This document was revised to include qualification of the "special case data" listed in Attachment VIII. 


\section{Data Qualification Report: Borehole Stratigraphic Contacts}

\section{Executive Summary}

The data set considered here is the borehole stratigraphic contacts data (DTN:

MO9811MWDGFM03.000) used as input to the Geologic Framework Model. A

Technical Assessment method used to evaluate these data with a two-fold approach: 1)

comparison to the geophysical logs on which the contacts were, in part, based; and 2)

evaluation of the data by mapping individual units using the entire data set. Qualification of the geophysical logs is being performed in a separate activity. A representative subset of the contacts data was chosen based on importance of the contact and representativeness of that contact in the total data set. An acceptance window was established for each contact based on the needs of the data users. Data determined to be within the acceptance window were determined to be adequate for their intended use in three-dimensional spatial modeling and were recommended to be Qualified. These methods were chosen to provide a two-pronged evaluation that examines both the origin and results of the data.

The result of this evaluation is a recommendation to qualify all contacts. No data were found to lie outside the pre-determined acceptance window. Where no geophysical logs are available, data were evaluated in relation to surrounding data and by impact assessment. These data are also recommended to be qualified. The stratigraphic contact data contained in this report (Attachment VII; DTN: MO0004QGFMPICK.000) are intended to replace the source data, which will remain unqualified.

\section{Introduction}

This qualification activity was performed in accordance with Data Qualification Plan: Borehole Stratigraphic Contacts (DI: TDP-NBS-GS-000001), which describes the scope, objectives, methods, QA procedures, and criteria for this activity.

This activity was performed in conjunction with other activities to qualify borehole data. The other activities are documented in the following reports:

- Borehole core samples: Data Qualification Report: Drill Core, Core Samples, Core Photos, Downhole Video, and Geophysical Logs from Boreholes, UE-25 a \#1, EU-25 a \#5, UE-25 a \#6, UE-25 a \#7, UE-25 b \#1, USG G-1, USGG-2, USG G-3, USG G-4, and USW GU-3 (DI: TDR-NBS-GS-000006)

- Geophysical Logs: Data Qualification Report: Composite Geophysical Logs (DI: TDR-NBS-GS-000005)

- Topographic grid: Data Qualification Report: Topographic Grid (DI: TDRNBS-GS-000004) 
Each step of this activity is documented in this report. This report is approved and signed by the qualification team. Qualifications of the team members are as follows:

\section{Robert W. Clayton}

Ph.D. in Geological Sciences, 8 years professional experience using geophysical logs and stratigraphic contacts data in subsurface exploration, including 6 years on the Yucca Mountain Project. Was not associated with acquisition of the contacts data.

\section{Clinton Lum}

Ph.D. in Geology, 7 years professional experience in Geology including 4 years on the Yucca Mountain Project. Was not associated with acquisition of the contacts data.

\section{Data Retrieval}

The borehole stratigraphic contacts data were requested and retrieved from the Technical Data Management System (TDMS). The data tracking number (DTN) is MO9811MWDGFM03.000. The data were provided on 3.5 inch floppy disk with transcription verification files and a copy of the Technical Data Information Form. The transmittal letter, floppy disk, and a printout of the data are included in Attachment I to this report. The data are in Excel spreadsheet format, and were verified against original copies of the spreadsheet obtained from TDMS at the time of original data submittal. The data appear to be original and accurate.

\section{Data Plotting}

The borehole stratigraphic contacts data were plotted on a suite of geophysical logs for conduct of this activity. The TDMS retrieval Excel spreadsheet was first edited for plotting by removing redundant contacts data. The redundant data were included in the spreadsheet to accommodate the requirements of $3 \mathrm{D}$ modeling software, which requires all cells filled to allow calculation of rock layer thicknesses (repeated contact depths are used to denote zero thickness). Cells coded as "not formed," "not present; faulted", or otherwise with repeated numbers were deleted. This editing step was verified during the qualification process as the plots were checked against the input data.

The contacts data were plotted on paper copies of the geophysical logs. While this could have been done by hand, to save time it was done using the plotting routine of QLA2/GES software, which was obtained from Configuration Management (CSCI 30005 V1.0). QLA2/GES software qualification is in progress, and the software was designated non-Q at the time of this report. However, plotting the stratigraphic contacts on the geophysical logs provided a rigorous validation of the plotting software because any errors would become obvious during examination of the data due to the inter-related nature of the contacts data and the level of detail at which the data were examined. No problems were found. The data qualification team concludes that the current qualification status of the plotting software does not affect the results of this data 
qualification because 1) the software plotting routine does not manipulate the data in any way, 2) the software was not used to develop or modify the data in any way, and 3) data qualification effectively validated the plotting software for this use.

Oversize color copies of the plots are being submitted with this report to the Records Information System, where the originals will be stored for future examination. The plots were also made available to the reviewers of this activity.

\section{Selection of Data to be'Examined}

Users of the Geologic Framework Model were queried to determine which contacts are most important to their analyses and the acceptance window (or tolerance) required for their analyses. The users queried are UZ Flow/Transport Model (Jennifer Hinds) and Repository Subsurface Design (Robert Elayer). The query letter and their responses are listed in Attachment II.

Based on the responses, a list of contacts was chosen to be examined as a) the most important contacts that appeared on both users' lists, and b) a representative sample of the contacts data. The required acceptance window (plus or minus) is also listed (the smallest of the acceptance windows provided by the users were used).

$\begin{array}{ll}\text { Tpcpv2/top of PTn } & 10 \mathrm{ft} . \\ \text { Tptrv1/top of TSw } & 10 \mathrm{ft} . \\ \text { Tptpv3/top of TSw3 } & 5 \mathrm{ft} . \\ \text { Tptpv2/top of CHn } & 15 \mathrm{ft} .\end{array}$

These are the most important contacts used in the GFM/ISM and downstream models. They are also the contacts where the acceptance window is most stringent, and so should provide a rigorous test of the contacts data and an indication of the applicability of these data to the GFM/ISM. The 5 meter acceptance window listed in Attachment II was rounded to 15 feet from the actual 16.4 feet for convenience in using the 10 foot grid interval on the geophysical logs plots (that is, a 15 foot window can be more easily determined visually than a 16.4 foot window).

Because all contacts were acquired using the same methods and based on the same types of data, the Data Qualification Team determined that a sub-sampling of the borehole contacts data is a reasonable and valid examination of the data set.

Attachment III is an oversize color cross section from the Geologic Framework Model version GFM3.1 through several boreholes. Geophysical logs are plotted at the borehole locations, except SD-6 for which no logs were available in the specialized format required for this type of plot. There is no vertical exaggeration in the cross section. The cross section demonstrates the large scale typical of the models which use the contacts data, and the relatively small impact of the acceptance windows. Because changing stratigraphic data by 15 feet (or more, in some cases) has negligible impact on the three- 
dimensional models, the Data Qualification Team concluded that the acceptance windows provided by the data users are reasonable and applicable to 3-D modeling.

Because no geophysical logs exist for the UZN series boreholes, these boreholes were not considered in this activity.

\section{Method of Examination}

The contacts data were evaluated by examining the plots of the contacts data for all boreholes side-by-side. Each contact was compared through the entire data set for consistency, geologic reasonability, and fit to geophysical curve signatures. The $\mathrm{Q}$ data from boreholes SD-6 and WT-24 were included in each comparison. In addition, maps were made of the thickness of the rock layer below each contact to provide an indication of the geospatial reasonability of each contact location. Because the thickness of each rock layer is inexorably linked to the stratigraphic package above and below it, thickness can be used as an indicator of the accuracy of a layer's top and bottom contact data. Thickness distribution of a layer should be reasonable in the context of the geologic setting. Thickness maps were also used because they were originally made as part of the Geologic Framework Model version GFM3.1 (DTN: MO9901MWDGFM31.000), and therefore provide a direct indication of the applicability of the contacts data to geospatial modeling. It should be noted, however, that the geologic setting at Yucca Mountain makes it difficult to evaluate the thickness maps because most units are highly variable in thickness and lithology from place to place. The thickness maps do provide, however, the only practical means to evaluate the contacts in their geospatial context, and are therefore a valuable tool. The thickness maps are included in Attachment IV.

Attachment $\mathrm{V}$ shows typical geophysical log signatures for each of the examined contacts (which are the boundaries between the rock layers above and below) and the expected placement of each contact. Borehole SD-12 was used as the standard because of its good quality logs. The plotted data were compared against this standard to determine whether the contact lies within the required acceptance window. Downhole conditions, log quality, lateral variations in lithology, and known local conditions were accounted for in making this determination.

Data that were determined to lie within the acceptance window were marked with a check $(\sqrt{ })$ on Attachment VI, the Contacts Data Examination Checklist. The checklist is the record of data evaluation by the data qualification team, and the plots of contacts on the geophysical logs (which are being submitted to the Records Information System concurrently with this report) are the record of what was examined. With these records and this report, the data qualification activity should be repeatable.

Data for which questions arose were marked with a question mark (?) in Attachment VI, and were compared to the thickness maps and data from surrounding boreholes for geologic reasonability, fit to thickness trends, and impact of borehole conditions. Anomalies in the thickness maps were examined to determine their origins as geologic or data-related. Data-related anomalies were further examined to determine whether the 
data should be accepted as qualified. If these examinations provided reasonable assurance that the contact is within the specified acceptance window, the contact was annotated with "okay" next to the question mark (?) on the Attachment VI checklist.

Contacts data found not to be within the required acceptance window were labelled with an X on the Attachment VI checklist. (NOTE: The results of this activity found no such data).

\section{Observed Log Signatures for Contacts}

The contacts shown in Attachment V are typical for the central Yucca Mountain site, but variations do occur due to geologic variability and local hole conditions.

The contact at the top of unit Tpcpv2 (top of PTn thermal-mechanical unit) is at the upper inflection point of a downward-decreasing, smooth ramp on the density log. In many locations, a low density break occurs near or above this contact (WT-24 is a good example). The key to defining rock layer Tpcpv2 is that it includes the slope on the density curve. The top contact also coincides with prominent breaks (abrupt changes from high to low plateaus) in the gamma and resistivity curves (a good example is SD-6).

The Tptrv1 rock layer (at the top of TSw) includes the first high density peak below the low-density PTn rocks. In most holes, this layer is only a few feet thick. Gamma and resistivity curves typically have prominent breaks at the top contact (good examples are WT-24 and SD-6).

The contact at the top of unit Tptpv3 (top of TSw3 vitrophyre) frequently has a low density zone at its top, typically related to borehole enlargement. In a few holes there are two or more additional low density (enlarged borehole) zones within the vitrophyre. The vitrophyre is characterized by relatively featureless log curves below the low density zones and to the lower density unit below (Tptpv2). Density values are typically at or above those of the overlying nonlithophysal zones. The top contact is usually (but not always) associated with prominent breaks in the gamma and resistivity curves (good examples are WT-24 and SD-6).

The contact at the top of unit Tptpv2 (base of vitrophyre, top of CHn) is similar to the contact at the top of unit Tpcpv2, because the Tptpv2 rock layer includes the density curve slope. The top contact of Tpcpv2 is the break in slope from the relatively featureless high density unit above (Tptpv3) to the low density unit below (Tptpv1).

\section{$\underline{\text { Results }}$}

All examined data were found to be within the required acceptance window. Table 1 shows that no data were found to be outside the acceptance window. This result is not surprising in view of the rigorous technical review the data have previously received. The 
contacts for which questions arose, but which were justified for $\mathrm{Q}$ recommendation are listed in Table 2, with justification.

The contacts recommended to be qualified are listed in Attachment VII. Special case contacts are listed in Attachment VIII. The special case contacts are those for which no geophysical logs are available, or where the geophysical logs are of so poor quality that they can not be used reliably to determine contacts. These data are discussed in the following section. The UZN series boreholes are not listed in the attachments because they were not considered in this activity.

\begin{tabular}{|l|l|l|l|}
\hline Borehole & Contact & Depth & Justification for non-Q recommendation \\
\hline -none- & -none- & & -none were found- \\
\hline
\end{tabular}

Table 1. Data recommended as non-Q.

\begin{tabular}{|l|l|l|l|}
\hline Borehole & Contact & Depth & Justification for Q recommendation \\
\hline b\#1 & Tptpv3 & 1283 & Gamma and Resistivity curves used instead of noisy Density curve \\
\hline H-3 & Tptpv3 & 1194 & Resistivity curve used instead of noisy Density curve \\
\hline H-4 & Tptpv3 & 1185 & Gamma and Resistivity curves used instead of noisy Density curve \\
\hline H-4 & Tptpv2 & 1209 & $\begin{array}{l}\text { This unit is the density transition on noisy Density curve, in accordance } \\
\text { with the definition of the unit }\end{array}$ \\
\hline
\end{tabular}

Table 2. Data requiring justification.

The Data Qualification Team determined that all of the examined contacts lie within the acceptance window. Because the remaining contacts data were acquired on a hole-byhole basis using the same methods as the data examined here, and because the acceptance windows for the other contacts are larger, it is anticipated that a similarly high percentage of the other contacts would be found to lie within the acceptance window.

Based on examination of the Attachment IV thickness maps, all examined contacts appear to be reasonable in their geologic and spatial context. The extreme thickness of layer Tpcpv2 in borehole WT-24 (Q data) was re-examined and determined to be reasonable. No other data anomalies were apparent. The anomalously thin Tptpv3 in borehole WT-1 is due to faulting, and so is acceptable. The Data Qualification Team concludes that thickness mapping of the units is a useful test of the contact data's adequacy for use in geoscientific modeling.

The recommendation to qualify these data is limited by the acceptance windows stated. Applications which require contacts to be less than the acceptance windows used here should evaluate the data in that context.

\section{Special Case Contacts Data}

For the contacts listed in Attachment VIII, no geophysical logs were available or the logs were of insufficient quality to be reliable in determining contact locations. These data 
were evaluated by two methods: 1) comparison to data in surrounding boreholes, and 2) impact assessment.

Comparison to surrounding data. All special case contacts were used in construction of the GFM, during which the data were rigorously compared to data in surrounding boreholes and in the context of the geologic system. No data were found to be anomalous or unacceptable. Examples of thickness maps using these data are found in Attachment IV and the GFM Analysis Model Report (MOL.20000121.0115, Figures 15 through 27). The methods used to map the data are discussed in the GFM Analysis Model Report section 6.3.

Impact assessment. The impacts of all special case contacts were evaluated, and the data were found to be acceptable based on the following rationale:

- Potential impacts of the special case contacts are small because users of the GFM either a) abstract (subsample) the GFM so that precise details are not preserved, or b) do not use data outside the immediate potential repository footprint and repository host horizons (all special case contacts are either outside the footprint or outside the potential repository host horizons [Tptpul through Tptpln]).

- Boreholes b\#1, G-2, H-6, J-13, NRG\#1, 2, 2b, 2c, 2d, and WT\#6 are not located in the potential repository area and therefore changes in stratigraphic contacts are unlikely to adversely impact analyses and models related to safety and waste isolation.

- All special case contacts were obtained by the same principal investigators as the data recommended to be qualified and using the same processes, procedures, and criteria; therefore, the data are unlikely to be unacceptable.

- The lack of anomalies and continuity of the special case data in comparison to surrounding data suggests that the contact elevations are constrained by the 15 foot ( 5 meter) acceptance window. This, and the fact that the special case data comprise less than $5 \%$ of the contacts suggest that the impacts of the special case data on the results of the GFM are small.

\section{Impacts of Data Qualification}

The contacts recommended to be qualified (Attachment VII; DTN:

MO0004QGFMPICK.000) comprise 100\% of the input contacts to the Geologic Framework Model version GFM3.1 (DTN: MO9901MWDGFM31.000), which is the primary customer for these data. The UZN series boreholes are not used as input to the GFM3.1, and were not considered in this activity. The source data (DTN: MO9811MWDGFM03.000) remain unqualified.

This qualification activity revealed no reason to suspect the technical quality of the borehole geophysical logs, the contacts, core, or other borehole data. 
TDR-NBS-GS-000007

Page 9 of 9

Signatures \& Dates

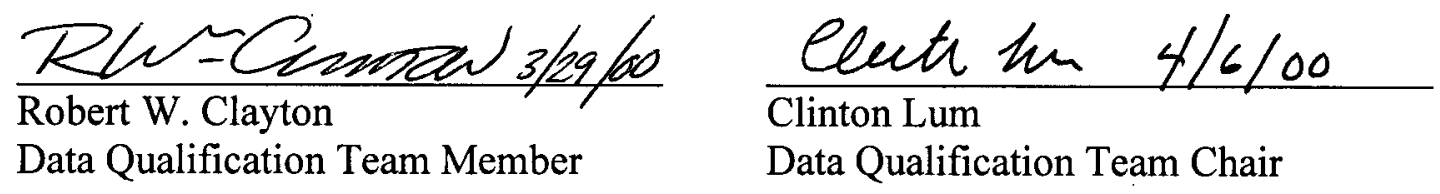

Ghert79 Went 04/10/00

Robert L. Howard

Responsible Manager

Deputy AMOPE 


\section{Attachment I:}

Data and Transmittal Letter from TDMS

p. I- 1 of 5

Plus attached floppy disk

28 page spreadsheet is numbered separately 


\subsection{5 .5400}

QA: N/A

Contract \# DE-AC08-91RW00134

LV.TDM.REK.07/99-029

July 14, 1999

Robert W. Clayton

M\&O/URSGWCFS

Yucca Mountain Project. Office

1261 Town Center Drive

Las Vegas, NV 89134

Dear Mr. Clayton:

Subject: Transmittal of GFM3.0 input data in support of data qualification activities.

DTN: MO9811MWDGFM03.000; TDIF: 307530

The enclosed 31/4" diskette contains a copy of the above referenced data. Also included on the diskette are the UNLX checksum and Message Digest Algorithm (MD5) results that may be used to verify the integrity of this file transfer.

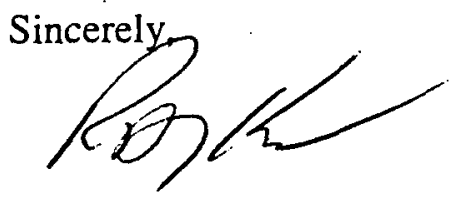

Raymond E. Keeler

MWD/SPA DBA

REK

Enclosure:

(1) Technical Data Information Form; 2 Pages

(2) File list; 1 Page

(3) $3 \frac{1}{4}$ " diskette $^{\prime \prime}$

cc w/o encl.:

S. J. Bodnar, M\&O, Las Vegas, Nevada, M/S 423 
$\square$ ACQUIRED DATA

DTN:

MO9811MWDGFM03.000

$\mathrm{X}$ DEVELOPED DATA

Preliminary Data:

\section{PART I Identification of Data}

Title of Data:

Description of Data: INPUT DATA TO GEOLOGTC FRAMEWORK MODEU, GEM3 O VA SUPPORTING DATA.

Data Originator/Preparer: BUESCH, D C

Data Originator/Preparer Organization: U.S. GEOLOGICAL SURVEY

Qualification Status: $\square \mathrm{Q} \square \mathrm{X}$ Un-Q $\square$ Accepted Governing Plan: SCP

SCP Activity Number(s): $\quad 8.3 .1 .4 .2 .1 .1$

WBS Number(s): $1 \cdot 2 \cdot 3 \cdot 2 \cdot 2 \cdot 1.1$

\section{PART II Data Acquisition/Development Information}

Method: EQUATING AND CORRELATING LITHOLOGIC FEATURES TO BOREHOLE GEOPHYSICAL LOGS.

Location(s): USGS, LAS VEGAS

Period(s): $\frac{5 / 5 / 1997 \text { to } 9 / 30 / 1997}{\text { From: } M M / D D N Y}$ To: MM/DDNY

Sample ID Number(s):

\section{PART III Source Data DTN(s)}

GS950708314211.033

GS960908312231.004

GS980108314211.001

\section{Comments}

INPUT DATA TO GEOLOGIC FRAMEWORK MODEL GFM3.0. SOME DATA WERE MODIFIED FROM DTN GS980108314211.001 FOR BOREHOLES NRG\#5 AND WT\#16 TO CORRECT TYPOGRAPHICAL ERRORS AND OMISSIONS. THESE DATA WERE REVIEWED IN

Checked by:

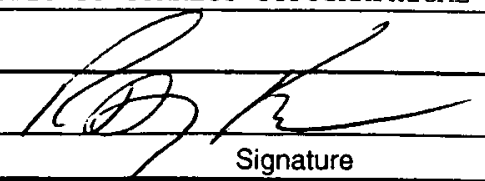

TMUSWSD1200095.001

TMUSWSD1200096.001 
YMP-023-R6 - YUCCA MOUNTAIN SITE CHARACTERIZATION PROJECT

$04 / 99$

TECHNICAL DATA INFORMATION

Page 2 of 2

Comments (continued)

ACCORDANCE WITH QUALITY ASSURANCE PROCEDURE QAP-SIII-2, REVIEW OF SCIENTIFIC DOCUMENTS AND DATA (THE

MODIFIED DATA WERE CORRECTED TO THE REVIEWED VALUES). THE DATA WERE ACQUIRED USING Q INFORMATION EXCEPT

SELECT INTERVALS IN BOREHOLES G-4, H-3, AND A\#4. 
Volume in arive $A$ is STRAT_PICKS

Directory of $\mathrm{A}: \backslash$

$\begin{array}{llrrr}\text { CHECKS 1 } & \text { TXT } & 70 & 07-14-99 & 7: 53 \text { a checksum results.txt } \\ \text { MD5_SI 1 } & \text { SIG } & 340 & 07-14-99 & 7: 53 \text { a md5_signature.sig } \\ \text { PIX98U 1 } & \text { XIS } & 226,816 & 07-14-99 & 7: 53 \text { a pix98usgs.xIs } \\ \text { M09811 - } & \text { HTM } & 148 & 07-14-99 & 7: 59 \text { a MO9811MWDGFM03_000.html } \\ & 4 \text { file(s) } & 227,374 \text { bytes } \\ & 0 \text { dir(s) } & 1,229,312 \text { bytes free }\end{array}$




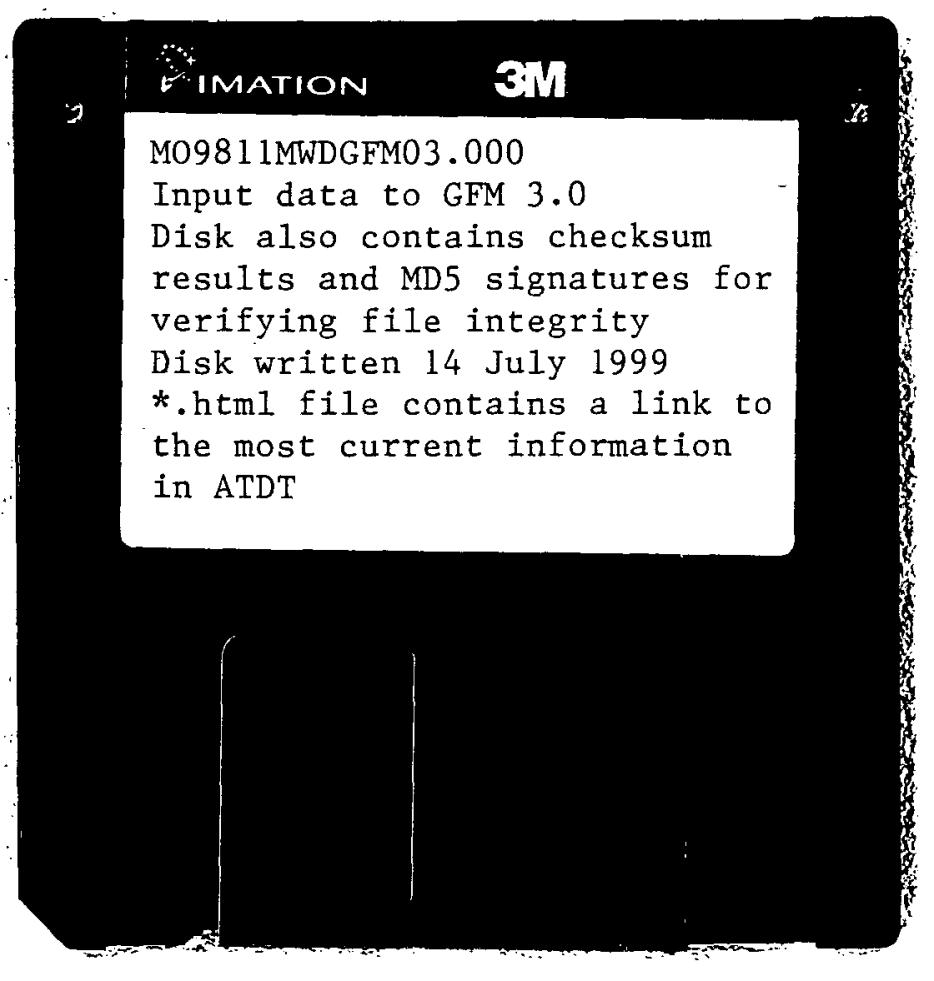




\begin{tabular}{|c|c|c|c|c|c|c|c|c|c|c|}
\hline & A & B & C & $\mathrm{D}$ & E & $\mathbf{F}$ & G & $\mathrm{H}$ & I & $\mathbf{J}$ \\
\hline 1 & Re-evaluation of key subsurface lithostratigraphic contacts: FY97 milestone SPG39IM & 4. Typosa & omissions correct & ted for input to & GFM3.0 by RW & V. Clayton. & & & & \\
\hline 2 & In addition to the lithostratigraphic contacts, the table contains values for the: & & & & & & & & & \\
\hline 3 & 1. vitric-zeolitic boundary (V-Z) & & Green cells & Resolution of $\mathrm{c}$ & ontacts initially cha & allenged by & view committee du & uring the $\mathrm{J}$ & ry $21-22,1998$, wo & orkshop. \\
\hline 4 & 2. total depth of each borehole & & Blue cells & Modified conta & cts based on sugge & estions by & on after review worl & ikshop in a & ion to re-examinatio & ion in the contex \\
\hline 5 & 3. type of contact at the top of each unit & & & & & & & & & \\
\hline 6 & Repository Host Horizon (RHH) identified by R. Elayer (MK M\&O) based on character of $\mathrm{g}$ & geophysical & & & & & & & & \\
\hline 7 & Colors of headers indicate: Black - Q-status geophysical logs (mostly) and core or cuttings & & & & & & & & & \\
\hline 8 & Blue - Q-status core or cuttings, no geophysical lo & & & & & & & & & \\
\hline 9 & & & & Contact & & Contact & & Contact & & Contact \\
\hline \begin{tabular}{|l|}
10 \\
11 \\
\end{tabular} & $\begin{array}{l}\text { Lithostrat unit } \\
\text { Not described }\end{array}$ & $\begin{array}{l}\text { Sumbol } \\
\mathrm{NC} \\
\end{array}$ & 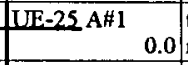 & $\begin{array}{l}\text { type } \\
\text { nd }\end{array}$ & 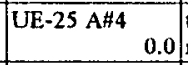 & $\begin{array}{l}\text { type } \\
\text { nd }\end{array}$ & \begin{tabular}{rl|} 
UE-25 A\#5 & \\
& 0.0 \\
\end{tabular} & $\begin{array}{l}\text { type } \\
\text { nd }\end{array}$ & $\begin{array}{rr}\text { UE-25 A\#6 } & \\
0.0 & \\
\end{array}$ & \begin{tabular}{|l} 
type \\
nd
\end{tabular} \\
\hline 12 & allurium & Qa & 0.0 & d & 0.0 & d & $0.0 \mid \mathrm{c}$ & d & 0.0 & d \\
\hline 13 & Rainier Mesa Tuff, includes pre-Rainier Mesa Tuff bedded tuff & Tmr & 30.0 & ne & 30.0 & ne & 90.0 .1 & ne & 20.0 & ne \\
\hline 14 & thyolite of Comb Peak & Tpk & 30.0 & ne & 30.0 & ne & 90.0 .1 & ne & & ne \\
\hline 15 & Tiva Canyon Tuff $(T p c)$ nondivided & Tpc_un & 30.0 & pe & 30.0 & pe & 90.0 .1 & pe & 20.0 & pe \\
\hline 16 & Tpc, crystal-poor vitric densely welded subzone & Tpcpv3 & 196.0 & nf & 119.0 & $n f$ & $128.0]$ & nf & 124.5 & 5 \\
\hline 17 & Tpc, crystal-poor vitric moderately welded subzone & Tpcpv2 & 196.0 & $c-v$ & 119.0 & $\mathrm{c}-\mathrm{v}$ & $128.0 \mathrm{C}$ & $\mathrm{c}-\mathrm{v}$ & 124.5 & $c-v$ \\
\hline 18 & Tpc, crystal-poor vitric nonwelded to partially welded subzones & Tpcpvl & 205.0 & $w$ & 135.0 & $w$ & 136.0 & $w$ & 135.0 & $w$ \\
\hline 19 & pre-Tiva Canyon Tuff bedded tuff & Tpbt4 & 210.0 & $d$ & 150.7 & d & 149.0 & d & 144.2 & $d$ \\
\hline 20 & Yucca Mountain Tuff nondivided & Tpy & 217.3 & nf & 154.3 & d & $155.0 \mathrm{c}$ & d & 149.3 & $d$ \\
\hline 21 & pre-Yucca Mountain Tuff bedded tuff & Tpbt3 & 217.3 & $d$ & 179.2 & $\mathrm{~d}$ & 164.5 & d & 167.0 & $d$ \\
\hline 22 & Pah Canyon Tuff nondivided & Tpp & 218.2 & $d$ & 197.0 & d & 180.0 & d & 186.0 & $d$ \\
\hline 23 & pre-Pah Canyon Tuff bedded tuff & Tpbt2 & 245.9 & $\mathrm{~d}$ & 273.6 & $d$ & 233.0 . & d & 201.5 & d \\
\hline 24 & Topopah Spring Tuff (Tpt) crystal-rich vitric nonwelded to partially welded zones & Tptrv3 & 266.8 & $\mathrm{~d}$ & 301.9 & $d$ & 262.0 & d & 229.8 & $d$ \\
\hline 25 & Tpt, crystal-rich vitric moderately welded zone & Tptrv2 & 273.0 & $\mathrm{w}$ & 309.0 & $w$ & 269.0 & $\mathbf{w}$ & 236.0 & $w$ \\
\hline 26 & Tph crystal-rich vitric densely welded zone & Tpirvl & 275.6 & $\mathbf{w}$ & 316.8 & $\mathrm{w}$ & $277.0 \mathrm{r}$ & nf & 241.7 & $\mathrm{w}$ \\
\hline 27. & Tpt crystal-rich nonlithophysal zone & Tptrm & 279.5 & $\mathrm{v}-\mathrm{c}$ & 317.0 & $\mathrm{v}-\mathrm{c}$ & 277.0 & $\mathrm{v}-\mathrm{c}, \mathrm{w}$ & 242.0 & $\mathrm{v}-\mathrm{c}$ \\
\hline 28 & Tph crystal-rich lithophysal zone & Tpurl & 409.8 & c & & np-td & 442.4 & & 402.0 & \\
\hline 29 & Tph, lithic-rich zone & Tptf & 438.0 & nf & & & 475.0 & nf & 422.0 & nf \\
\hline 30 & Tpt crystal-poor upper lithophysal zone & Tptpul & 438.0 & c & & & 475.0 & & 422.0 & \\
\hline 31 & Tpt, crystal-poor middle nonlithophysal zone & Tptpmn & 673.0 & c & & & & np-td & & np-td \\
\hline 32 & Tpt, crystal-poor lower lithophysal zone & Tptpll & 745.0 & c & & & & & & \\
\hline 33 & Tpt crystal-poor lower nonlithophysal zone & Tptpln & 1084.0 & c & & & & & & \\
\hline 34 & Tpt, crystal-poor vitric densely welded subzone & Tptpv3 & 1271.6 & $\mathrm{c}-\mathrm{v}$ & & & & & & \\
\hline 35 & Tpt crystal-poor vitric moderately welded subzone & Tptpv2 & 1310.1 & $w$ & & & & & & \\
\hline 36 & Tpt, crystal-poor virric nonwelded to partially welded subzones & Tptpv1 & 1324.6 & $\mathrm{w}$ & & & & & & \\
\hline 37 & pre-Topopah Spring Tuff bedded tuff & Tpbtl & 1360.0 & $\mathrm{az}$ & & & & & & \\
\hline 38 & Calico Hills Formation undifferentiated & Tac & 1368.6 & az & & & & & & \\
\hline 39 & pre-Calico Hills Formation bedded tuff & Tacbt & 1789.3 & $\mathrm{az}$ & & & & & & \\
\hline 40 & Prow Pass Tuff (Tcp) upper virric(zeolitic) nonwelded to partially welded zones & Tepuv & 1832.2 & $\mathrm{az}$ & & & & & & \\
\hline 41 & Tcp, upper crystallized nonwelded to partially welded zones & Tсpuc & 1845.1 & $\begin{array}{ll}-c & (a z-c)\end{array}$ & & & & & & \\
\hline 42 & Tсp, crystallized moderately to densely welded zones & Tcpm & 1944.0 & $c, w$ & & & & & & \\
\hline 43 & Tcp, lower crystallized nonwelded to partially welded zones & Tcplc & 2006.0 & $c, w$ & & & & & & \\
\hline 44 & Tcp, lower vitric(zeolitic) nonwelded to partially welded zones & Tcplv & 2030.0 & $c-y \quad a z(c-a z)$ & & & & & & \\
\hline 45 & pre-Prow Pass Tuff bedded tuff & Tcpbt & 2331.4 & $\mathrm{az}$ & & & & & & \\
\hline 46 & Bullfrog Tuff (Tcb) upper vitric(zeolitic) nonwelded to partially welded zones & Tcbuv & 2333.2 & np-e & & & & & & \\
\hline 47 & Tcb, upper crystallized nonwelded to partially welded zones & Tcbuc & 2333.2 & pe $\quad(a z-c)$ & & & & & & \\
\hline 48 & Tcb, crystallized moderately to densely welded zones & Tcbm & 2415.0 & $c, w$ & & & & & & \\
\hline 49 & Tcb, lower crystallized nonwelded to partially welded zones & Tcblc & & np-td & & & & & & \\
\hline 50 & Tob, lower vitric(zeolitic) nonwelded to partially welded zones & Tcblv & & & & & & & & \\
\hline 51 & pre-Bullfrog Tuff bedded tuff & Tcbbt & & & & & & & & \\
\hline 52 & Tram Tuff (Tct) upper vilric(zeolitic) nonwelded to partially welded zones & Tcluv & & & & & & & & \\
\hline
\end{tabular}




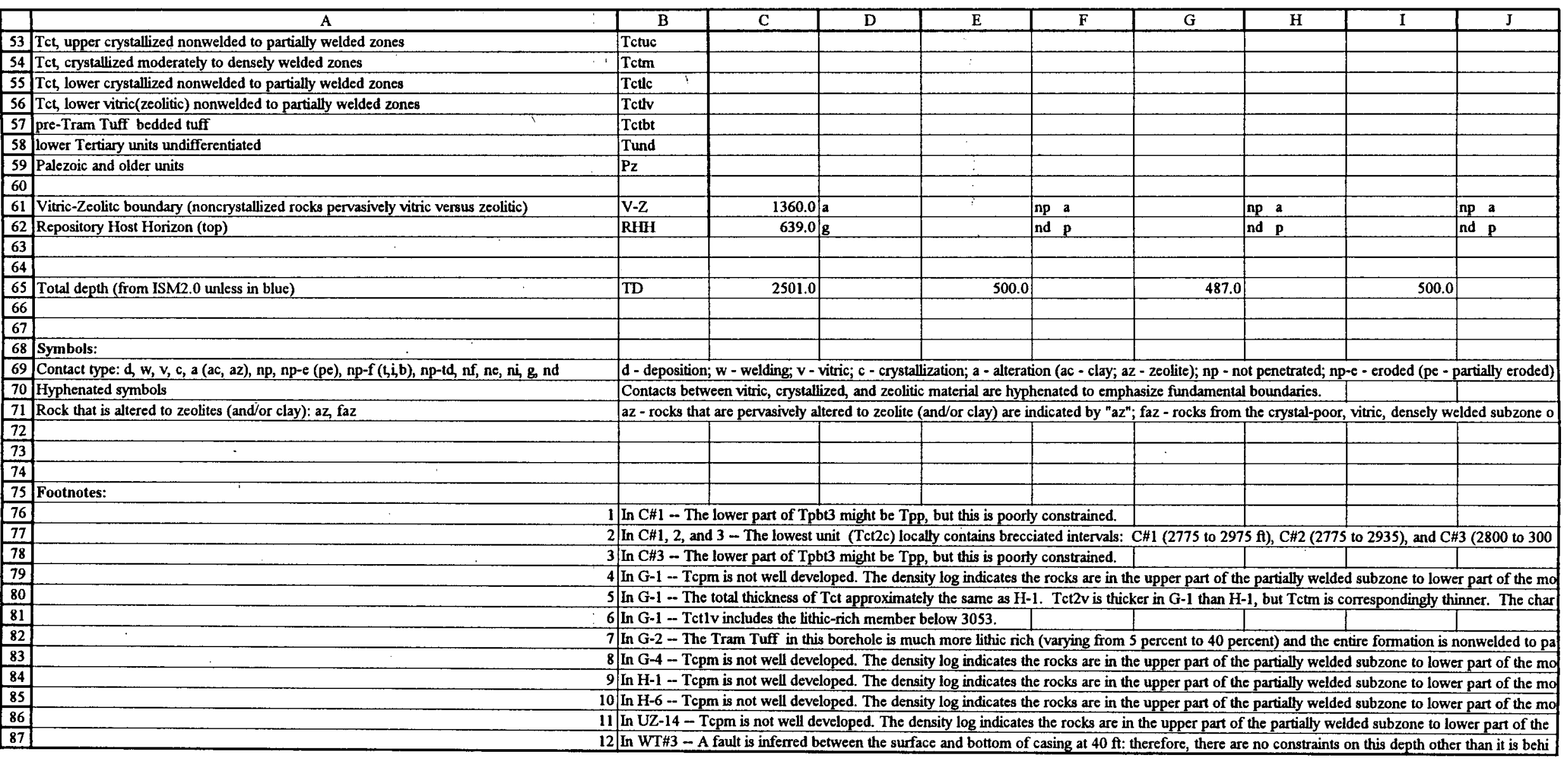




\begin{tabular}{|c|c|c|c|c|c|c|c|c|c|c|c|c|c|c|}
\hline & $\mathrm{K}$ & $\mathrm{L}$ & $\mathbf{M}$ & $\mathrm{N}$ & 0 & $P$ & $Q$ & $\mathrm{R}$ & $S$ & $T$ & $\mathbf{U}$ & $\mathrm{V}$ & $\bar{W}$ & $\bar{x}$ \\
\hline 1 & & & & & & & & & & & & & & \\
\hline 2 & & & & & & & & & & & & & & \\
\hline 3 & & & & & & & & & & & & & & \\
\hline 4 & of committee chall & ilenges. & & & & & & & & & & & & \\
\hline 5 & & & & & & & & & & &. & & & \\
\hline 6 & & & & & & & & & & & & & & \\
\hline 7 & & & & & & & & & & & & & & \\
\hline 8 & & & & & & & & & & & & & & \\
\hline 9 & & Contact & & Contact & & Contact & & Contact & & Contact & & Contact & & Contact \\
\hline \begin{tabular}{|l|}
10 \\
11 \\
\end{tabular} & \begin{tabular}{|r|}
$U E-25 \mathrm{~A} \# 7$ \\
0.0
\end{tabular} & $\begin{array}{l}\text { type } \\
\text { nd }\end{array}$ & $\frac{U E-25 \mathrm{BH}}{0.0}$ & $\begin{array}{l}\text { nope } \\
\text { nd }\end{array}$ & UE-25CH1 & one & 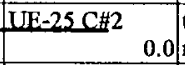 & $\begin{array}{l}\text { type } \\
\text { nd }\end{array}$ & 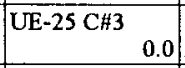 & \begin{tabular}{|l} 
type \\
nd
\end{tabular} & $\begin{array}{rr}\text { USW G-1 } & \\
& 0.0 \\
& \\
& \\
\end{array}$ & $\begin{array}{l}\text { type } \\
\text { nd }\end{array}$ & \begin{tabular}{|l|l|} 
USW G-2 & \\
& 0.0 \\
\end{tabular} & $\begin{array}{l}\text { type } \\
\text { nd }\end{array}$ \\
\hline 12 & 0.0 & & 0.0 & d & 0.0 & $n \dot{e}$ & 0.0 & $d$ & 0.0 & $d$ & 0.0 & $d$ & & $n p$ \\
\hline 13 & 165.0 & ne & $156.0 \mathrm{t}$ & & 0.0 & ne & 69.9 & ne & 80.1 & ne & $60.0 \mathrm{n}$ & ne & & ne \\
\hline 14 & 165.0 & ne & 156.0 & & 0.0 & ne & 69.9 & ne & 80.1 & ne & 60.0 & ne & & ne \\
\hline 15 & 165.0 & pe & 156.0 & & 60.0 & pe & 69.9 & pe & 80.1 & pe & 60.0 & pe & & pe \\
\hline 16 & 170.0 & nf & 180.0 & & 251.0 & $\mathrm{nf}$ & 243.0 & $\mathrm{nf}$ & 221.0 & $\mathrm{nf}$ & 60.0 & np-e & 225.0 & $\mathrm{nf}$ \\
\hline 17 & 170.0 & $c-v$ & 180.0 & & 251.0 & $c-v$ & 243.0 & $\hat{c}-\mathrm{v}$ & 221.0 & $\mathrm{c}-\mathrm{v}$ & 60.0 & np-e & 225.0 & $c-v$ \\
\hline 18 & 175.8 & $w$ & 182.0 & & 266.0 & $w$ & 257.0 & & 238.0 & $w$ & 60.0 & np-e & 228.0 & $\mathrm{w}$ \\
\hline 19 & 190.0 & d & 189.0 & & 271.0 & $d$ & 264.0 & & 247.0 & $d$ & 60.0 & np-e & 235.0 & $d$ \\
\hline 20 & 194.2 & & $192.5 \mathrm{r}$ & & 274.0 & $\mathrm{nf}$ & 267.0 & & 250.0 & nf & 60.0 & pe & 245.0 & $d$ \\
\hline 21 & 212.0 & d & 192.5 & & 274.0 & (1) & 267.0 & d & 250.0 & (3) & 102.0 & & 341.5 & $d$ \\
\hline 22 & 226.5 & $d$ & 204.5 & & 300.0 & nf & 286.0 & $\mathrm{nf}$ & 271.0 & nf & 135.0 & & 494.2 & d \\
\hline 23 & 266.7 & d & 243.0 & & 300.0 & $d$ & 286.0 & & 271.0 & $d$ & 235.0 & & 730.8 & $d$ \\
\hline 24 & 291.9 & d & 259.0 & & 319.0 & $d$ & 306.0 & d & 286.0 & $d$ & 265.0 & $\mathrm{nf}$ & 755.2 & $d$ \\
\hline 25 & 303.6 & $\mathbf{w}$ & 267.0 & $\mathbf{w}$ & 327.0 & $w$ & 313.0 & $w$ & 295.0 & $w$ & 265.0 & & 761.7 & $w$ \\
\hline 26 & 304.5 & $\mathrm{w}$ & $275.0 \mathrm{v}$ & $\mathbf{w}$ & 329.0 & $w$ & 315.0 & $w$ & 298.0 & $w$ & 270.0 & $w$ & 766.8 & $w$ \\
\hline 27 & 311.0 & $v-c$ & 279.9 & $\mathrm{v}-\mathrm{c}$ & 332.0 & $\mathrm{v}-\mathrm{c} \quad(\mathrm{fb}-435)$ & 318.0 & $\mathrm{v}-\mathrm{c}$ & 303.0 & $v-c$ & 280.0 & $\mathrm{v}-\mathrm{c}$ & 771.2 & $y=c$ \\
\hline 28 & 483.0 & & 413.0 & & 424.0 & $\mathrm{ft}$ & 423.0 & & 400.0 & & 438.0 & & 909.1 & $\mathrm{nf}$ \\
\hline 29 & 508.0 & nf & 440.0 .1 & & 438.0 & nf & 457.0 & & 438.0 & & $456.51:$ & nf & 909.1 & d \\
\hline 30 & 508.0 & & $440.0 \mathrm{C}$ & & 438.0 & $c$ & 457.0 & & 438.0 & & 456.5 & & 977.2 & d \\
\hline \begin{tabular}{|l|l|}
31 \\
\end{tabular} & 770.0 & & 680.5 & & 595.0 & $\mathrm{c}$ & 591.0 & & 563.0 & $\mathrm{ft}$ & 713.4 & & 1246.0 & c \\
\hline \begin{tabular}{|l|l|}
32 \\
\end{tabular} & 878.8 & & $765.0 \mathrm{~d}$ & & 726.0 & & 725.0 & & 703.0 & & $814.8 \mathrm{C}$ & & 1280.0 & $c$ \\
\hline \begin{tabular}{|l|}
33 \\
\end{tabular} & & np-td & $1130.0 \mathrm{c}$ & & 1040.0 & c & 1038.0 & & 1030.0 & & $1199.2 \mathrm{C}$ & $c$ & 1604.0 & c \\
\hline 34 & & & 1283.0 & faz & 1216.0 & faz & 1205.0 & $c-v$ & 1183.0 & $c-v$ & 1287.0 & $\mathrm{c}-\mathrm{v}$ & 1633.8 & $c-\mathrm{v}$ \\
\hline 35 & & & $1336.0 \mathrm{v}$ & $\mathrm{az}$ & 1293.0 & $\mathrm{az}$ & 1290.0 & $\mathrm{az}$ & 1270.0 & az & $1342.4 \mathrm{v}$ & $\mathrm{w}$ & 1670.0 & az \\
\hline 36 & & & $1352.0 \mathrm{v}$ & $\mathrm{az}$ & 1320.0 & $a z$ & 1320.0 & $\mathrm{az}$ & 1298.0 & $\mathrm{az}$ & 1360.5 & $\mathrm{w}>1394.3 \mathrm{az}$ & 1684.5 & $\mathrm{az}$ \\
\hline 37 & & & 1374.0 & az & 1334.0 & nf & 1335.0 & nf & 1320.0 & nf & $1403.9 \mathrm{~d}$ & d $\quad a z$ & 1702.1 & $a z$ \\
\hline 38 & & & $1385.0 \mathrm{~d}$ & $a z$ & 1334.0 & az & 1335.0 & $\mathrm{az}$ & 1320.0 & $\mathrm{az}$ & 1425.5 & $\mathrm{az}$ & 1757.0 & $\mathrm{az}$ \\
\hline 39 & & & $1845.0 \mathrm{~d}$ & az & 1581.0 & $\mathrm{az}$ & 1580.0 & $\mathrm{az}$ & 1580.0 & $a z$ & $1736.4 \mathrm{~d}$ & $a z$ & 2576.7 & $a z$ \\
\hline 40 & & & $1882.0 \mathrm{p}$ & & 1692.0 & np-e & 1658.0 & np-e & 1635.0 & np-e & 1799.0 & $\mathrm{az}$ & 2704.7 & np-e \\
\hline 41 & & & 1896.0 & $v-c$ & 1692.0 & $v-c$ pe az $(a z-c)$ & 1658.0 & $v-c$ pe $a z(a z-c)$ & 1635.0 & $\mathrm{v}-\mathrm{c}$ pe az $(\mathrm{az}-\mathrm{c})$ & 1862.5 & $\mathrm{v}-\mathrm{c}$ az $(\mathrm{az}-\mathrm{c})$ & 2704.7 & np-e \\
\hline 42 & & & $1992.0 \mathrm{c}$ & $c, w$ & 1787.0 & $c, w$ & 1773.0 & $c, w$ & 1762.0 & $c, w$ & 1920.0 & $c, w \quad(4)$ & 2704.7 & pe \\
\hline \begin{tabular}{|l|}
43 \\
\end{tabular} & & & $2039.0 \mathrm{C}$ & $c, w$ & 1863.0 & $c, w$ & 1849.0 & $c, w$ & 1838.0 & $c, w$ & 1960.0 & $c, w$ & $2963.7 \mathrm{C}$ & $c, w$ \\
\hline 44 & & & $2071.0 \mathrm{c}$ & $c-v a z(c-a z)$ & 1884.0 & $\mathrm{c}-\mathrm{v} \quad \mathrm{az}(\mathrm{c}-\mathrm{az})$ & 1872.0 & $c-v \quad a z(c-a z)$ & 1863.0 & $\mathrm{c}-\mathrm{v} \quad \mathrm{az}(\mathrm{c}-\mathrm{az})$ & $1985.7 \mathrm{C}$ & $c-v \quad a z(c-a z)$ & 2980.0 & $\mathrm{c}-\mathrm{v} \quad$ az $(\mathrm{c}-\mathrm{az})$ \\
\hline 45 & & & 2355.6 & az & 2119.0 & $\begin{array}{ll}\mathrm{d} & \mathrm{az} \\
\end{array}$ & 2109.5 & $\mathrm{az}$ & 2110.0 & $\mathrm{az}$ & 2154.9 & $\mathrm{az}$ & 3246.5 & az \\
\hline 46 & & & 2361.3 & np-e & 2153.0 & d $\quad a z$ & $2138.0 \mathrm{C}$ & $\mathrm{az}$ & 2130.0 & $\mathrm{az}$ & 2173.2 & $\mathrm{az}$ & 3281.9 & $\mathrm{az}$ \\
\hline 47 & & & 2361.3 & pe $\quad(a z-c)$ & 2240.0 & $\mathrm{v}-\mathrm{c} \quad \mathrm{az}(\mathrm{az}-\mathrm{c})$ & 2227.0 & $\mathrm{v}-\mathrm{c}$ az $(\mathrm{az}-\mathrm{c})$ & 2218.0 & $\mathrm{v}-\mathrm{c}$ az $(\mathrm{az}-\mathrm{c})$ & 2337.0 & $\mathrm{v}-\mathrm{c} \quad \mathrm{az}(\mathrm{az}-\mathrm{c})$ & 3302.5 & $\begin{array}{ll}v-c & (a z-c) \\
\end{array}$ \\
\hline 48 & & & $2468.0 \mathrm{c}$ & $c, w$ & 2275.0 & $c, w$ & 2262.0 & $c, w$ & 2267.0 & $c, w$ & $2461.0 \mathrm{c}$ & $c, w$ & 3320.0 & $c, w$ \\
\hline 49 & & & $2782.8 \mathrm{c}$ & $c, \mathbf{w}$ & 2446.0 & $c, w$ & 2445.0 & c, w & 2428.0 & $c, w$ & $2547.0 \mathrm{n}$ & & $3447.0 \mathrm{c}$ & $c, w$ \\
\hline 50 & & & $2799.5 \mathrm{c}$ & $\mathrm{c-v}$ az (c-az) & $2575.0 \mathrm{~s}$ & $c-v \quad a z(c-a z)$ & $2550.0 \mathrm{c}$ & $c-v \quad a z(c-a z)$ & 2547.0 & $c-v \quad a z(c-a z)$ & $2547.0 \mathrm{c}$ & $c-v_{2}, w$ & $3485.0 \mathrm{c}$ & $c-v \quad$ az $(c-a z)$ \\
\hline 51 & & & $2852.7 \mathrm{~d}$ & $\mathrm{az}$ & 2692.0 & $\mathrm{az}$ & 2667.0 & $\mathrm{az}$ & 2670.0 & & 2601.6 & az & \begin{tabular}{|l|l}
3503.4 & $\mathrm{~d}$ \\
\end{tabular} & $\mathrm{az}$ \\
\hline 52 & & & $2882.5] \mathrm{d}$ & $\mathrm{az}$ & 2754.0 & $n p-\varepsilon$ & 2725.0 & np-e & $2704.0_{11}$ & np-e & $2639.4 \mathrm{~d}$ & $\mathrm{az}$ & $3574.0 \mathrm{n}$ & (7) \\
\hline
\end{tabular}




\section{-}

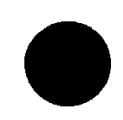

p. $1-4$ or 28

\begin{tabular}{|c|c|c|c|c|c|c|c|c|c|c|c|c|c|c|c|}
\hline & $\mathrm{K}$ & $\bar{L}$ & $\bar{M}$ & $\mathbf{N}$ & 0 & $P$ & $\mathrm{Q}$ & $\mathbf{R}$ & $\mathrm{s}$ & $\mathrm{T}$ & $\mathrm{U}$ & $\mathrm{V}$ & $\mathrm{w}$ & & $\bar{x}$ \\
\hline 53 & & & 2933.0 & $\begin{array}{ll}\mathrm{V}-\mathrm{c} & (\mathrm{az}-\mathrm{c})\end{array}$ & 2754.0 & pe $\quad(a z-c)(2)$ & 2725.0 & $p e(a z-c)(2)$ & 2704.0 & $p e(a z-c)(2)$ & 2800.0 & $v-c$ az $(a z-c)$ & 3574.0 & $\operatorname{nf}$ & (7) \\
\hline 54 & & & 3158.0 & $c, w$ & & np-td & & np-td & & np-td & 2840.0 & $c, w$ & 3574.0 & nf & (7) \\
\hline 55 & & & 3322.0 & $c, w$ & & & & & & & 2956.0 & $c, w$ & 3574.0 & nf & (7) \\
\hline 56 & & & 3359.9 & $\mathrm{c}-\mathrm{v}$ az (c-az) & & & & & & & 3005.0 & $c-v$ az (6) & 3574.0 & $d$ & $\mathrm{az}$ \\
\hline \begin{tabular}{|l|}
57 \\
\end{tabular} & & & 3900.9 & d az & & & & & & & 3522.0 & az & 3914.0 & $d$ & $\mathrm{az}$ \\
\hline 58 & & & 3960.3 & $\mathrm{az}$ & & & & & & & 3558.2 & $\mathrm{az}$ & 3982.0 & $d$ & $\mathrm{az}$ \\
\hline 59 & & & & np-td & & & & & & & & np-td & & np-id & \\
\hline \multicolumn{16}{|l|}{60} \\
\hline 61 & & np a & 1336.0 & $a$ & 1293.0 & $a$ & 1290.0 & $a$ & 1270.0 & $a$ & 1394.3 & $a$ & 1670.0 & $\mathbf{a}$ & \\
\hline 62 & & nd $p$ & 632.0 & $\mathrm{~g}$ & 515.0 & $\mathrm{~g}$ & 520.0 & $g$ & 500 & $g$ & 600.0 & $\mathrm{~g}$ & 1131.9 & $\mathrm{~g}$ & \\
\hline \multicolumn{16}{|l|}{63} \\
\hline \multicolumn{16}{|l|}{64} \\
\hline 65 & 1002.0 & & 4003.0 & & 3000.0 & & 3000.0 & & 3000.0 & & 6000.0 & & 6602.0 & & \\
\hline \multicolumn{16}{|l|}{66} \\
\hline \multicolumn{16}{|l|}{67} \\
\hline \multirow{2}{*}{\multicolumn{16}{|c|}{ 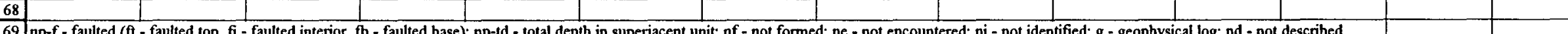 }} \\
\hline \multicolumn{9}{|c|}{69 np-f - faulted (ft - faulted top, fi - faulted interior, fb - faulted base); np-td - total depth in superjacent unit; nf - not formed; ne - not encountered; ni - not identified; $\mathrm{g}$ - geophysical log; nd - not described } & & & & & & & \\
\hline 70 & & & & & & 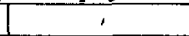 & & & & & & & & & \\
\hline 71 & \multicolumn{15}{|c|}{ the Topopah Spring Tuff that have significant alteration along (typically high-angle) fractures } \\
\hline \multicolumn{16}{|c|}{72} \\
\hline \multicolumn{16}{|l|}{73} \\
\hline \multicolumn{16}{|l|}{74} \\
\hline \multicolumn{16}{|l|}{75} \\
\hline 76 & & & & & & i & & & & & & & & & \\
\hline 77 & (total depth]). & & & & & & & & & & & & & & \\
\hline \multicolumn{16}{|l|}{78} \\
\hline \multirow{2}{*}{\multicolumn{16}{|c|}{$\begin{array}{l}79 \text { eratcly welded subzone and crystallized. The Tcpm unit could be modeled as absent in this location if unit density and calculated porosi } \\
00 \text { cter of the upper part of Tctm and possibly lower part of } \mathrm{Tct} 2 \mathrm{c} \text { appear to differ from the typical profile. }\end{array}$}} \\
\hline & & & & & & & & & & & & & & & \\
\hline 81 & & & & & & & & & & & & & & & \\
\hline 82 & \\
\hline 83 & \multirow{2}{*}{\multicolumn{14}{|c|}{$\begin{array}{l}\text { erately welded subzone and crystallized. The Tcpm unit could be modeled as absent in this location if unit density and calculated porosity are the dominant modeling parameters. } \\
\text { erately welded subzone and crystallized. The Tcpm unit could be modeled as absent in this location if unit density and calculated porasity are the dominant madeling prateter }\end{array}$}} & \\
\hline 84 & & & & & & & & & & & & & & & \\
\hline 85 & \multicolumn{15}{|c|}{ erately welded subzone and crystallized. The Tcpm unit could be modeled as absent in this location if unit density and calculated porosity are the dominant modeling parameters. } \\
\hline 86 & \multirow{2}{*}{\multicolumn{15}{|c|}{$\begin{array}{l}\text { oderately welded subzone and crystallized. The Tcpm urit could be modeled as absent in this location if unit density and calculated porosity are the dominant modeling parameters. } \\
\text { casing. }\end{array}$}} \\
\hline 87 & & & & & & & & & & & & & & & \\
\hline
\end{tabular}




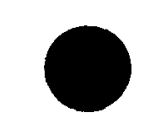

\begin{tabular}{|c|c|c|c|c|c|c|c|c|c|c|c|c|c|c|}
\hline & $\mathrm{Y}$ & $\mathrm{Z}$ & $\overline{A A}$ & $\mathrm{AB}$ & $\mathrm{AC}$ & $\mathrm{AD}$ & $\mathrm{AE}$ & $\mathrm{AF}$ & $\overline{A G}$ & $\mathrm{AH}$ & $\mathrm{AI}$ & $\mathrm{AJ}$ & $\overline{A K}$ & $\mathrm{AL}$ \\
\hline 1 & & & & & & & & & & & & & & \\
\hline 2 & & & & & & & & & & & & & & \\
\hline 3 & & & & & & & & & & & & & & \\
\hline 4 & & & & & & & & & & & & & & \\
\hline 5 & & & & & & & & & & & & & & \\
\hline 6 & & & & & & & & & & & & & & \\
\hline 7 & & & & & & & & & & & & & & \\
\hline 8 & & & & & & & & & & & & & & \\
\hline 9 & & Contact & & Contact & 4 & Contact & & Contact & & Contact & & Contact & & Contact \\
\hline 10 & USW GU-3/G-3 & lype & USW G-4 & ndipe & \begin{tabular}{|l|l|} 
USWH-1 & \\
0.0 & 1
\end{tabular} & inge & 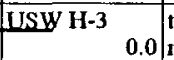 & $\begin{array}{l}\text { type } \\
\text { nd }\end{array}$ & $\begin{array}{llll}\text { USW H}^{-4} & \\
& 0.0\end{array}$ & $\begin{array}{l}\text { type } \\
\text { nd }\end{array}$ & $\begin{array}{lll}\text { USW H-5 } & & \\
& 0.0 & t\end{array}$ & $\begin{array}{l}\text { type } \\
\text { nd }\end{array}$ & $\mid \begin{array}{ll}\text { USW H-6 } & \\
& 0.0\end{array}$ & $\begin{array}{l}\text { lype } \\
\text { nd }\end{array}$ \\
\hline 12 & 0.0 & ne & 0.0 & d & 0.0 .1 & ne & 0.0 & ne & 0.0 & ne & 0.01 & ne & 0.0 & d \\
\hline 13 & 0.0 & ne & 30.0 & ne & 0.0 .5 & ne & $0.0 \mathrm{r}$ & ne & 0.0 & ne & 0.01 & ne & 29.9 & ne \\
\hline 14 & 0.0 & ne & 30.0 & ne & $0.0: \mathrm{I}$ & ne & $0.0 \mathrm{I}$ & ne & 0.0 & ne & $-\quad 0.0 \mathrm{I}$ & ne & 29.9 & ne \\
\hline 15 & 0.0 & pe & 30.0 & pe & 0.0 & pe & 0.0 & pe & 0.0 & $\mathrm{pe}$ & 0.0 & $\mathrm{pe}$ & 29.9 & pe \\
\hline 16 & 348.1 & & 118.0 & $\mathrm{nf}$ & $61.0 \mathrm{~s}$ & $\mathrm{nf}$ & 369.8 & $c-v$ & 173.9 & nf & 0.0 .1 & nf & 190.0 & $\mathrm{nf}$ \\
\hline 17 & 357.0 & & 118.0 & $c-v$ & 61.0 & $c-v$ & 376.0 & & 173.9 & $c-v$ & 390.0 & $\mathrm{c}-\mathrm{v}$ & 190.0 & $\mathrm{c}-\mathrm{v}$ \\
\hline 18 & 368.4 & & 130.0 & $\mathbf{w}$ & 70.0 & $w$ & $388.0 \mathrm{~V}$ & $w$ & 185.0 & $w$ & 415.0 & $w$ & 200.1 & $w$ \\
\hline 19 & 372.5 & & 141.0 & d & 90.0 & $d$ & 400.0 & $d$ & 193.0 & & 437.5 & d & 260.2 & $d$ \\
\hline 20 & 375.5 & & 146.0 & d & $95.1 \mathrm{C}$ & d & $403.9 \mathrm{r}$ & nf & 202.0 & $\mathrm{nf}$ & 438.0 & d & 270.0 & d \\
\hline 21 & 375.5 & & 148.8 & $d$ & 160.8 & & 403.9 & & 202.0 & & 457.0 & $d$ & 275.0 & $d$ \\
\hline 22 & 391.7 & & 168.2 & $d$ & 190.3 & & 417.0 & nf & 221.0 & nf & 471.0 & d & 278.0 & $d$ \\
\hline 23 & 391.7 & & 198.9 & d & 278.9 & $d$ & 417.0 & & 221.0 & $d$ & 510.0. & $d$ & 290.0 & $d$ \\
\hline 24 & 417.7 & & 224.0 & nf & 295.0 & $d$ & 435.0 & $d$ & 243.5 & d & 542.0 & $d$ & 300.2 & $d$ \\
\hline 25 & 424.9 & & 236.5 & $w$ & 320.0 & $w$ & 441.9 & $w$ & 248.5 & & 560.0 & $w$ & 330.0 & $w$ \\
\hline 26 & $427.8 \mathrm{I}$ & & 239.0 & $w$ & 330.0 & $w$ & 445.0 & $w$ & 251.0 & & 562.0 & $w$ & 330.0 & $w$ \\
\hline 27 & 427.8 & $v-c, w$ & 242.8 & $v-c$ & 335.0 & v-c (fb) & 449.0 & $\mathrm{v}-\mathrm{c}$ & 254.0 & $v-c$ & 564.0 & $v-c$ & 332.0 & $\mathrm{v} \cdot \mathrm{c}$ \\
\hline 28 & 542.0 & & 400.4 & $\mathrm{c}$ & 505.0 & f & 526.9 & & 376.0 & $\operatorname{nf}(?)$ & 700.0 & c & 409.0 & c \\
\hline 29 & 548.0 & & 420.0 & nf & 538.0 & $n f$ & 540.0. & nf & 376.0 & & 741.0 . & nf & 435.0 & $\mathrm{nf}$ \\
\hline 30 & 548.0 & & 420.0 & $\mathrm{c}$ & 538.0 & & 540.0 & & 376.0 & & 741.0 & $\mathrm{c}$ & 435.0 & c \\
\hline 31 & 688.0 & & 674.0 & c & 788.0 & & 680.1 & & 576.0 & & $988.0 \mathrm{C}$ & c & 653.0 & c \\
\hline 32 & 830.0 & & 774.0 & c & 897.0 & & 848.110 & c & 703.0 & & $1088.0 \mathrm{c}$ & c & 795.0 & $c$ \\
\hline 33 & 1044.0 & & 1127.9 & c & 1324.0 & & $1049.9 \mathrm{c}$ & & 987.0 & & 1450.0 & c & 1097.0 & $c$ \\
\hline 34 & $1186.7 \mathrm{C}$ & & 1316.5 & $\mathrm{c}-\mathrm{v}$ & 1410.0 & $\mathrm{c}-\mathrm{v}$ & $1194.0 \mathrm{c}$ & $c-v$ & 1185.0 & $c-v$ & $1582.0 \mathrm{C}$ & $\mathrm{c}-\mathrm{v}$ & 1213.0 & $c-v$ \\
\hline 35 & 1280.0 & & 1345.4 & $\underline{w}$ & 1469.5 & $w$ & 1308.0 & w & 1209.0 & & 1659.0 & $w$ & 1310.0 & $w$ \\
\hline 36 & 1317.0 & & 1353.6 & $\mathrm{w}>1376.0 \mathrm{az}$ & 1486.2 & $w>1490.0 \mathrm{az}$ & $1341.0 \mathrm{O}$ & $\mathbf{w}$ & 1247.0 & & 1672.0 & w & 1322.0 & $w$ \\
\hline 37 & 1406.3 & & 1406.8 & $\mathrm{az}$ & 1498.0 & & 1392.0 & & 1312.0 & & $1699.1 \mathrm{~d}$ & d & 1356.0 & np-e? \\
\hline 38 & 1412.5 & & 1409.4 & $a z$ & 1505.0 & $\mathrm{az}$ & 1400.0 & & 1317.0 & $d>1330.0 \mathrm{az}$ & 1705.0 & d & 1356.0 & $\mathrm{~d}>1429.0 \mathrm{az}$ \\
\hline 39 & 1506.3 & & 1705.4 & $\mathrm{az}$ & 1802.0 & $\mathrm{az}$ & $1437.0 \mathrm{c}$ & & 1572.0 & $\mathrm{az}$ & 1879.9 & $\mathrm{az}$ & 1458.0 & az \\
\hline 40 & $1553.9 \mathrm{~d}$ & & 1762.7 & $a z$ & 1861.0 & $\mathrm{az}$ & $1495.0 \mathrm{~d}$ & & 1626.9 & $\overline{a z}$ & 1944.9 & $a z$ & 1508.0 & $a z$ \\
\hline 41 & 1597.0 & $\mathrm{v}-\mathrm{c}$ & 1793.6 & $\mathrm{v}-\mathrm{c} \quad(\mathrm{az}-\mathrm{c})$ & 1911.0 & $v-c \quad(a z-c)$ & $1518.0 \mathrm{v}$ & $v-c$ & 1662.0 & $y-c \quad(a z-c)$ & $1967.0 \mathrm{~V}$ & \begin{tabular}{|l|l|}
$v-c$ & $(a z-c)$ \\
\end{tabular} & 1555.0 & $\begin{array}{ll}v-c & (a z-c)\end{array}$ \\
\hline 42 & $1663.0, \mathrm{c}$ & $c, w$ & 1880.0 & $c, w \quad(8)$ & 1969.0 & $c, w$ & $1640.0 \mathrm{C}$ & $c, \mathbf{w}$ & 1746.0 & $c, w$ & $2085.0 \mathrm{c}$ & $c, w$ & 1602.0 & c, $w \quad(10)$ \\
\hline 43 & $1744.0 \mathrm{C}$ & $c, w$ & 1946.0 & $c, w$ & 2021.0 & $c, w$ & $1690.0 \mathrm{c}$ & $c, w$ & 1820.0 & $c, w$ & $2113.0 \mathrm{C}$ & $c, w$ & 1670.0 & $c, w$ \\
\hline 44 & $1755.0 \mathrm{C}$ & $c-v>1816.0 \mathrm{az}$ & 1954.6 & $\mathrm{c}-\mathrm{v} \quad \mathrm{az}(\mathrm{c}-\mathrm{az})$ & 2053.0 & $c-v$ az $(c-a z)$ & $1702.0 \mathrm{C}$ & $c-v>1762$ az & 1840.0 & $c-v$ az (c-az) & $2130.0 \mathrm{c}$ & $c-v \quad a z$ (c-az) & 1685.0 & $c-v \quad a z(c-a z)$ \\
\hline 45 & $1992.3 \mathrm{c}$ & $\mathrm{az}$ & 2238.0 & $\mathrm{az}$ & 2300.0 & $\mathrm{az}$ & $1899.9 \mathrm{~d}$ & $\mathrm{az}$ & 2263.1 & $\mathrm{az}$ & $2240.1 \mathrm{~d}$ & $\mathrm{az}$ & 1765.1 & $a z$ \\
\hline 46 & 1998.7 & $\mathrm{az}$ & 2245.7 & $a z$ & 2319.5 & $\mathrm{az}$ & $1907.1 \mathrm{~d}$ & $\mathrm{az}$ & 2274.9 & $\mathrm{az}$ & $2263.1 \mathrm{~d}$ & $a z$ & 1794.9 & $\mathrm{az}$ \\
\hline 47 & 2021.3 & $v-c \quad(a z-c)$ & 2255.0 & $y-c \quad(a z-c)$ & 2337.0 & $v-c \quad(a z-c)$ & 1922.0 & $v-c \quad(a z-c)$ & 2369.0 & $y-c \quad(a z-c)$ & 2310.0 & $v-c \quad(a z-c)$ & 1881.0 & \begin{tabular}{|l|l|}
$v-c$ & $(a z-c)$ \\
\end{tabular} \\
\hline 48 & $2102.0 \mathrm{c}$ & $c, w$ & 2560.0 & $c, w$ & 2533.0 & $c, w$ & $2092.0 \mathrm{c}$ & $c, w$ & 2494.0 & $c, w$ & $2388.0 \mathrm{c}$ & $c, w$ & 1894.0 & $c, w$ \\
\hline 49 & $2549.5 \mathrm{c}$ & $c, w$ & 2677.0 & nf & 2629.0 & $c, w$ & 2350.0 & $c, w$ & 2559.0 & $c, w$ & 2468.0 c & $c, w$ & $1990.0] \mathrm{c}$ & $\mathrm{c}, \mathbf{w}$ \\
\hline $50 \mid$ & $2550.8 / \mathrm{c}$ & $c-v \quad a z(c-a z)$ & 2677.0 & $c-v w$ az $(c-a z)$ & 2676.0 & $c-v a z(c-a z)$ & $2397.0 \mathrm{c}$ & $\mathrm{c}-\mathrm{v} \quad \mathrm{az}(\mathrm{c}-\mathrm{az})$ & 2635.0 & $c-v$ az $(c-a z)$ & $2510.0 \mathrm{c}$ & $c-v \quad a z(c-a z)$ & 2138.1 & $c-v \quad$ az $(c-a z)$ \\
\hline 51 & $2617.0 \mathrm{~d}$ & $\mathrm{az}$ & 2733.3 & $\mathrm{az}$ & 2690.3 & $a z$ & 2449.10 & $\mathrm{az}$ & 2644.0 & $\mathrm{az}$ & $2712.9 \mathrm{~d}$ & d $\quad$ az & 2225.0 & $3 z$ \\
\hline 52 & $2637.0 \mathrm{~d}$ & $\mathrm{az}$ & 2755.6 & $\mathrm{az}$ & 2729.6 & $\mathrm{az}$ & 2477.0 & $\mathrm{az}$ & 2664.0 & a2 & $2742.11 \mathrm{~d}$ & $\mathrm{az}$ & 2258.0 & $\mathrm{az}$ \\
\hline
\end{tabular}



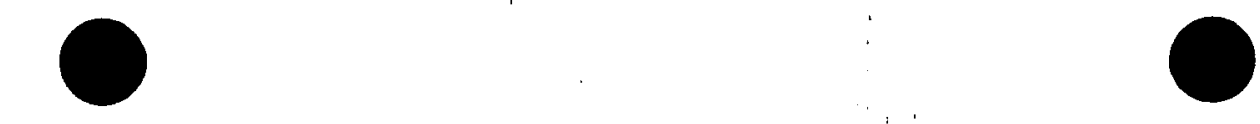

\begin{tabular}{|c|c|c|c|c|c|c|c|c|c|c|c|c|c|c|}
\hline & $Y$ & $\mathrm{Z}$ & $\mathbf{A A}$ & $\mathrm{AB}$ & $\mathrm{AC}$ & $\mathrm{AD}$ & $\mathrm{AE}$ & $\mathrm{AF}$ & $\mathrm{AG}$ & $\mathrm{AH}$ & AI & $\mathrm{AJ}$ & $\mathrm{AK}$ & AL \\
\hline 53 & 2719.0 & $v-c \quad(a z-c)$ & 2839.0 & $v-c \quad(a z-c)$ & 2823.0 & $v-c \quad(a z-c)$ & 2567.0 & $v-c \quad(a z-c)$ & \multicolumn{2}{|c|}{$2745.0[\mathrm{v}-\mathrm{c} \quad(\mathrm{az}-\mathrm{c})$} & 2845.0 & $y-c \quad(a z-c)$ & 2348.0 & \multirow{2}{*}{$\frac{v-c \quad(a z-c)}{c, w}$} \\
\hline 54 & 2890.0 & $c, w$ & 2950.0 & $c, w$ & 2862.0 & $c, w$ & 2692.0 & $c, w$ & \multicolumn{2}{|c|}{$2835.0 \mathrm{c}, \mathrm{w}$} & 2897.0 & $\begin{array}{l}\mathrm{V}-\mathrm{c} \quad(\mathrm{az}-\mathrm{c}) \\
c, \mathrm{w}\end{array}$ & 2439.0 & \\
\hline 55 & 3265.0 & $c, w$ & & np-td & 3073.0 & $c, w$ & 3086.0 & $c, w$ & 3200.0 & $c, w$ & 3130.0 & $c, w$ & 2655.0 & $c, w$ \\
\hline 56 & 3290.0 & $c-\mathrm{v} \quad \mathrm{az}(\mathrm{c}-\mathrm{az})$ & & & 3111.0 & $c-v$ az $(c-a z)$ & 3120.0 & $\mathrm{c}-\mathrm{v}$ az $(\mathrm{c}-\mathrm{az})$ & 3228.0 & $\mathrm{c}-\mathrm{v}$ az $(\mathrm{c}-\mathrm{az})$ & 3150.0 & $c-v \quad a z(c-a z)$ & 2667.0 & $c-v$ az $(c-a z)$ \\
\hline 57 & 3850.1 & az & & & 3618.7 & $\mathbf{a z}$ & 3595.1 & az & 3788.0 & $\mathrm{az}$ & 3412.0 & az & 2869.1 & az \\
\hline 58 & 3876.3 & $\mathrm{az}$ & & & 3661.4 & $\mathrm{az}$ & 3637.1 & az & 3818.9 & az & 3421.9 & $\mathrm{az}$ & 2877.9 & $\mathrm{az}$ \\
\hline 59 & & inp-td & & & & np-ld & & np-td & \multicolumn{2}{|r|}{ np-td } & & np-td & & np-td \\
\hline \multicolumn{15}{|l|}{60} \\
\hline 61 & 1816.0 & $\mathbf{a}$ & 1376.0 & $\mathrm{a}$ & 1490.0 & $\mathbf{a}$ & 1762.0 & $\mathrm{a}$ & \multicolumn{2}{|c|}{$1330.0 \mathrm{a}$} & 1888.0 & \multirow{2}{*}{$\frac{a}{g}$} & 1429.0 & \multirow{2}{*}{0.2} \\
\hline 62 & 675.0 & $g$ & 618.0 & $\mathrm{~g}$ & 650.0 & $\mathrm{~g}$ & 605.0 & $\mathrm{~g}$ & \multicolumn{2}{|c|}{$553.0 \mathrm{~g}$} & 931.0 & & 585.0 & \\
\hline \multirow{2}{*}{\multicolumn{14}{|c|}{$\frac{63}{64}$}} & $0 . \mathrm{g}$ \\
\hline \multicolumn{11}{|l|}{64} & & & & \\
\hline 65 & 5031.0 & & 3001.0 & & 6000.0 & & 4000.0 & & 4004.0 & & 4000.0 & & 4002.0 & \\
\hline \multirow{2}{*}{\multicolumn{15}{|c|}{$\begin{array}{l}66 \\
67\end{array}$}} \\
\hline & & & & & & & & & & & & & & \\
\hline \multicolumn{15}{|l|}{68} \\
\hline \multirow{2}{*}{\multicolumn{15}{|c|}{$\begin{array}{l}69 \\
70\end{array}$}} \\
\hline 70 & & & & & & & & & & & & & & \\
\hline \multicolumn{15}{|l|}{71} \\
\hline \multirow{2}{*}{\multicolumn{15}{|c|}{\begin{tabular}{|l|}
72 \\
73
\end{tabular}}} \\
\hline 73 & & & & & & & & & & & & & & \\
\hline 74 & & & & 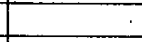 & & & & & & & & & & \\
\hline 75 & & & & & & & & & & & & & & \\
\hline 76 & & & & & & & & & & & & & & \\
\hline 77 & & & & & & & & & & & & & & \\
\hline 78 & & & & & & & & & & & & & & \\
\hline 79 & & & & & & & & & & & & & & \\
\hline 80 & & & & & & & & & & & & & & \\
\hline 81 & & & & & & & & & & & & & & \\
\hline 82 & & & & & & & & & & & & & & \\
\hline 83 & & & & & & & & & & & & & & \\
\hline 84 & & & & & & & & & & & & & & \\
\hline 85 & & & & & & & & & & & & & & \\
\hline 86 & & & & & & & & & & & & & & \\
\hline 87 & & & & & & & & & & & & & & \\
\hline
\end{tabular}




\begin{tabular}{|c|c|c|c|c|c|c|c|c|c|c|c|}
\hline & $\overline{\mathrm{AM}}$ & AN & AO & AP & $\overline{\mathrm{AQ}}$ & $\overline{A R}$ & AS & AT & $\overline{\mathrm{AU}}$ & AV & $\overline{A W}$ \\
\hline 1 & & & & & & & & & & & \\
\hline 2 & & & & & & & & & & & \\
\hline 3 & & & & & & 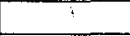 & & & & & \\
\hline 4 & & & & & & & & & & & \\
\hline 5 & & & & & & & & & & & \\
\hline 6 & & & & & & & & & & & \\
\hline 7 & &. & & & & & & & & & \\
\hline 8 & & & & & & & & & & & \\
\hline 9 & & Contact & & Contact & & Contact & & Contact & & Contact & \\
\hline$\frac{10}{11}$ & 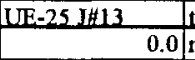 & $\begin{array}{l}\text { tope } \\
\text { nd }\end{array}$ & UE-25 SRGHA & Tupe & \begin{tabular}{|c|}
$\mathrm{NE}-25 \mathrm{NRG} \# 2$ \\
0.0
\end{tabular} & type & 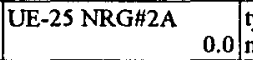 & $\begin{array}{l}\text { type } \\
\text { nc }\end{array}$ & 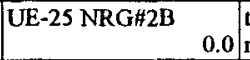 & $\begin{array}{l}\text { type } \\
\text { nd }\end{array}$ & $\begin{array}{ll}\text { UE-25 NRG } \# 2 C & \\
& 0.0\end{array}$ \\
\hline 12 & 0.0 & & 9.5 & nf & 0.0 & np & $80.6 \pi$ & $\mathrm{ni}$ & $0.0 \mathrm{I}_{\mathrm{I}}$ & ne & 50.0 \\
\hline 13 & $435.0 \mathrm{r}$ & & $9.5 \mathrm{r}$ & ne & 0.0 & ne & $80.6 \mathrm{n}$ & ni & $2.2 \mathrm{r}$ & $n p-e$ & 50.0 \\
\hline 14 & 435.0 & & 9.5 & ne & 164.6 & ne & 80.6 & d & $157.3 \mathrm{C}$ & $d$ & 150.1 \\
\hline 15 & 435.0 & & 9.5 & $\mathrm{pe}$ & 164.6 & & 165.9 & $\overline{p e}$ & 232.3 & f & \\
\hline 16 & 587.0 & $c-v$ & & np-td & 276.3 & nf & & np-td & 232.3 & $f$ & \\
\hline 17 & 591.0 & $w$ & & & 276.3 & $c-v$ & & & 232.3 & f & \\
\hline 18 & 610.0 & $w$ & & & 282.8 & $\mathrm{w}$ & & & 232.3 & $f$ & \\
\hline 19 & 629.0 & & & & & np-td & & & 265.2 & d & \\
\hline 20 & 632.0 & nf & & & & & & & 267.1 & $d$ & \\
\hline 21 & 632.0 & d & & & & & & & 268.9 & $d$ & \\
\hline 22 & 650.0 & nf & & & & & & & 285.3 & d & \\
\hline 23 & 650.0 & d & & & & & & & 324.0 & d & \\
\hline 24 & 682.0 & d & & & & & & & & $\operatorname{np}-\mathrm{td}$ & \\
\hline 25 & 686.0 & $w$ & & & & & & & & & \\
\hline 26 & 691.0 & nf & & & & & & & & & \\
\hline 27 & 691.0 & & & & & & & & & & \\
\hline 28 & 755.0 & & & & & & & & & & \\
\hline 29 & 801.01 & & & & & & & & & & \\
\hline 30 & 801.0 & & & & & & & & & & \\
\hline 31 & 905.0 & & & & & & & & & & \\
\hline 32 & $1003.0 \mathrm{c}$ & & & & & & & & & & \\
\hline 33 & $1193.0 \mathrm{C}$ & & & & & & & & & & \\
\hline 34 & $1300.0 \mathrm{c}$ & $\begin{array}{ll}c-v & f a z \\
\end{array}$ & & & & & & & & & \\
\hline 35 & $1415.0 \mathrm{v}$ & $a z$ & & & & & & & & & \\
\hline 36 & $1450.0 \mathrm{y}$ & $\mathrm{az}$ & & & & & & & & & \\
\hline 37 & 1475.0 & az & & & & & & & & & \\
\hline 38 & 1482.0 & az & & & & & & & & & \\
\hline 39 & $1682.0 \mathrm{~d}$ & az & & & & & & & & & \\
\hline 40 & 1711.0 & az & & & & & & & & & \\
\hline 41 & 1742.0 & v-c $\quad(a z-c)$ & & & & & & & & & \\
\hline 42 & 1848.0 & $c, w$ & & & & & & & & & \\
\hline 43 & 1942.0 & $c, w$ & & & & & & & & & \\
\hline 44 & $1961.0 \mathrm{C}$ & $\mathrm{c}-\mathrm{v}$ az $(\mathrm{c}-\mathrm{az})$ & & & & & & & & & \\
\hline 45 & 1993.0 & $\mathrm{az}$ & & & & & & & & & \\
\hline 46 & $2017.0 \mathrm{n}$ & np-e & & & & & & & & & \\
\hline 47 & 2017.0 & $v-c \quad(a z-c)$ & & & 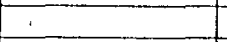 & & & & & & \\
\hline 48 & $2082.0 \mathrm{c}$ & $c, w \quad f b$ & & & & & & & & & \\
\hline 49 & $2322.0 \mathrm{n}$ & $n p-f$ & & & & & & & & & \\
\hline 50 & $2322.0 \mathrm{n}$ & np-f & & & & & & & & & \\
\hline 51 & $2322.0 \mathrm{f}$ & & & & & $\therefore$ & & & & & \\
\hline 52 & $2358.0 \mid \mathrm{d}$ & d $\quad a z$ & & & & & & & & & \\
\hline
\end{tabular}




\section{-}
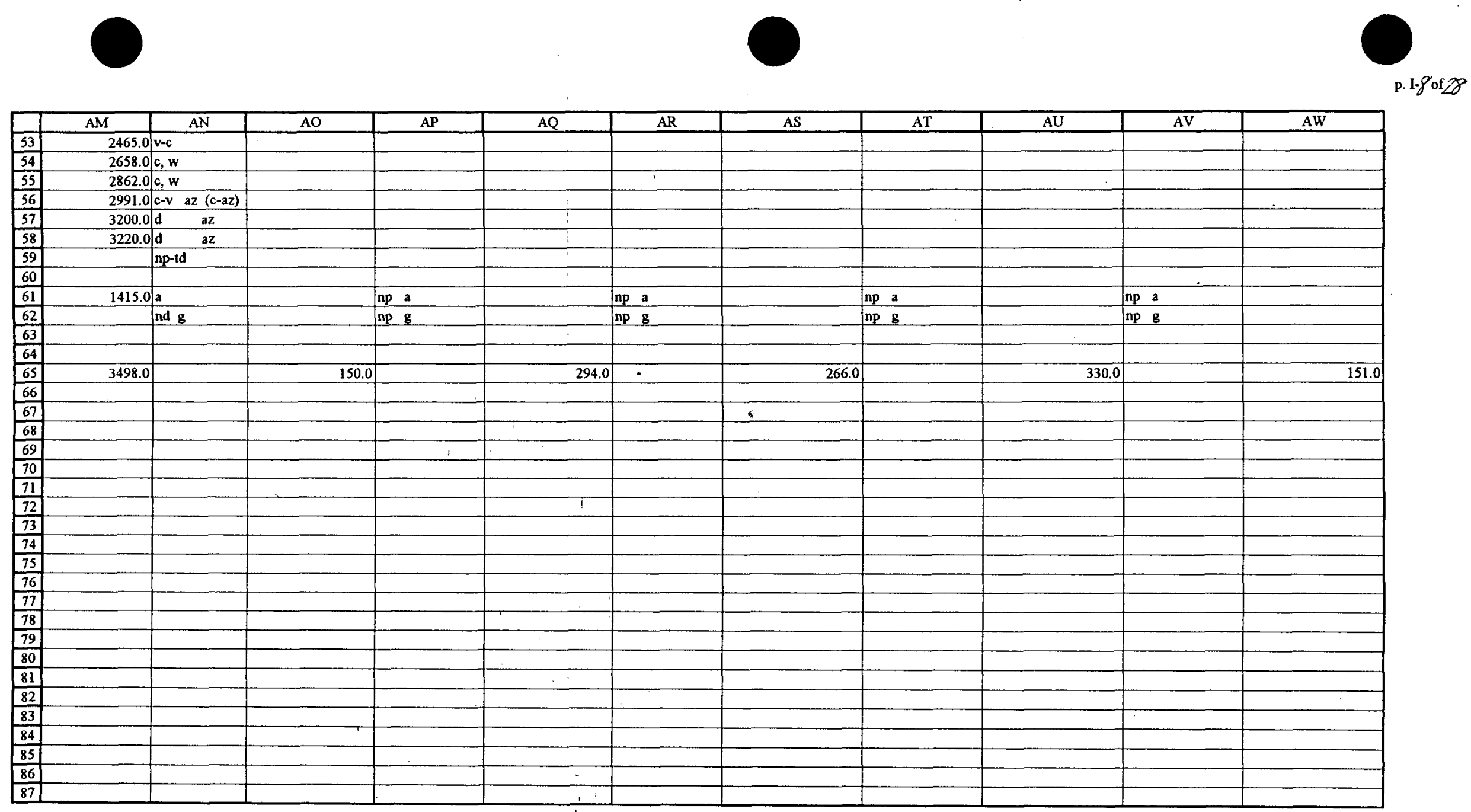


\section{$\bullet$}

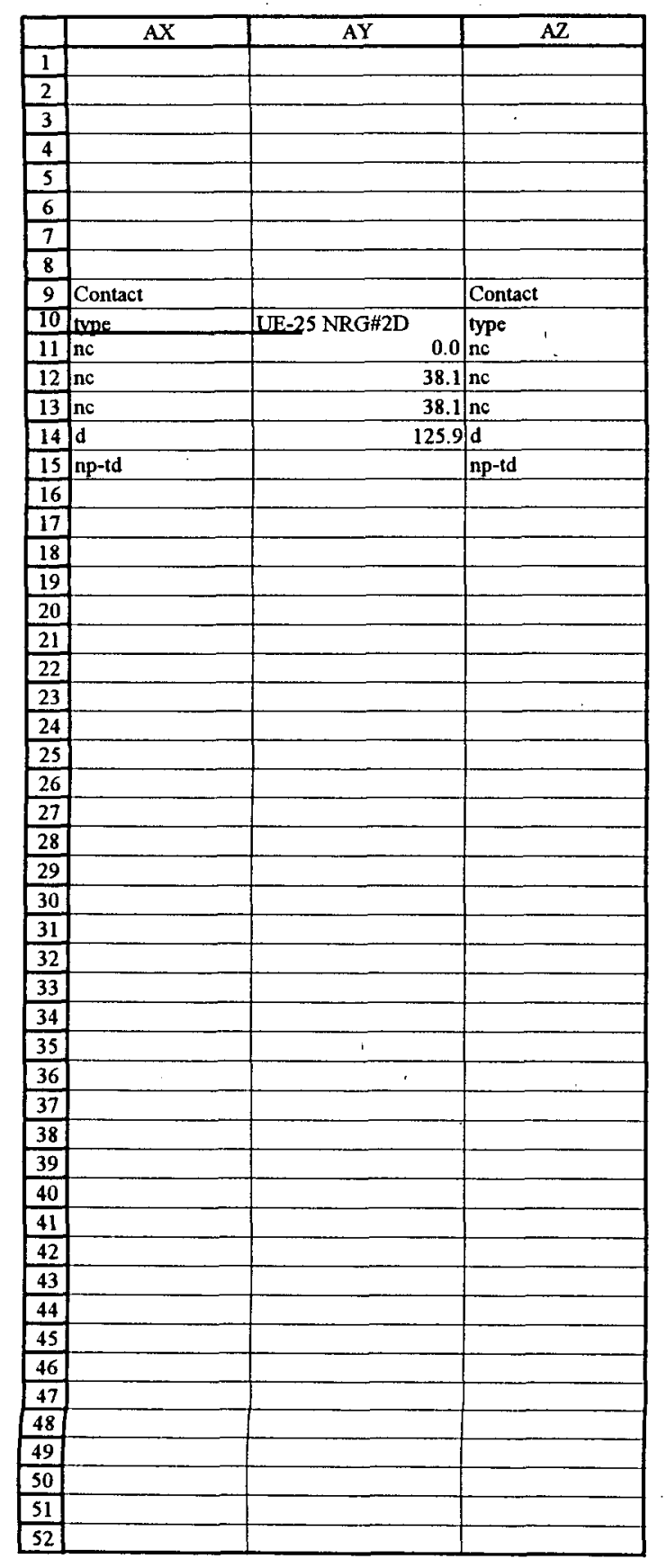



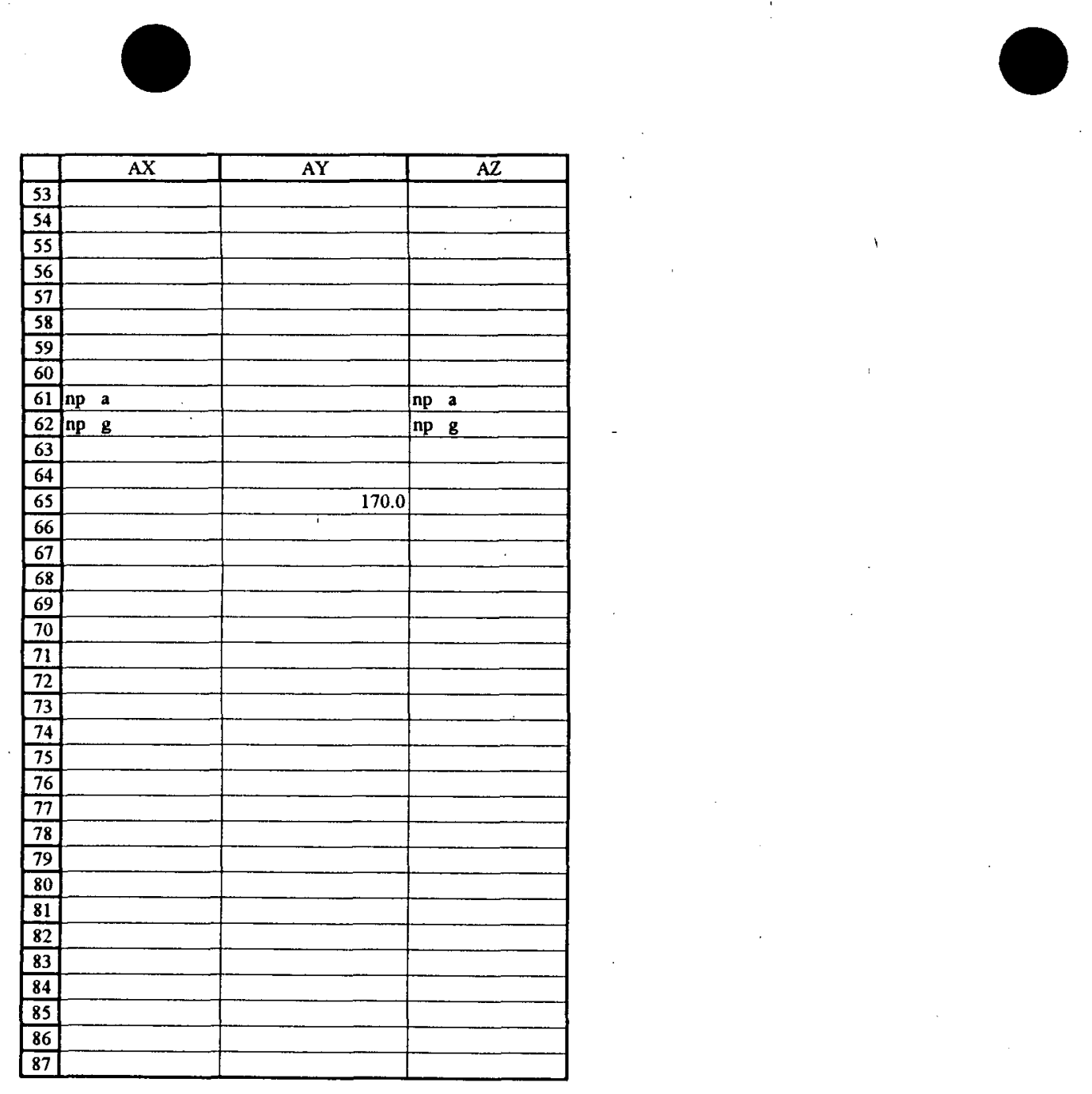


\begin{tabular}{|c|c|c|c|c|c|c|c|c|c|c|c|c|}
\hline & BA & $\mathrm{BB}$ & $\mathrm{BC}$ & $\mathrm{BD}$ & $\mathrm{BE}$ & $\mathrm{BF}$ & BG & $\mathrm{BH}$ & BI & BJ & BK & $\mathrm{BL}$ \\
\hline 1 & & & & & & & & & & & & \\
\hline 2 & & . & & & & & & & & & & \\
\hline 3 & & & & & & $i$ & & & & & & \\
\hline 4 & & & & & & & & & & & & \\
\hline 5 & & & & & & & & & & & & \\
\hline 6 & & & & & & & & & & & & \\
\hline 7 & & & & & & & & & & & & \\
\hline 8 & & & & & & & & & & & & \\
\hline 9 & & & Contact & & Contact & & Contact & & Contact & & Contact & \\
\hline 10 & Symbol & UE-25NRGH3 & tspe & UR:-25NRGH4 & tope & UE-25NRGA5 & type & USW NRG-6 & type & USW NRG-7/7A & type & $\mathrm{ONC} \# 1$ \\
\hline 11 & $\mathrm{NC}$ & 0.0 & nd & 0.0 & nd & 0.0 & nd & $0.0]_{1}$ & nd & 0.0 & nc & 0.0 \\
\hline 12 & $\mathrm{Qa}$ & 0.0 & $\mathrm{np}$ & 0.0 & nf & 0.0 & ne & 0.0 & ne & $\left.17.0\right|_{x}$ & ne & 0.0 \\
\hline 13 & $\operatorname{Tm} r$ & 0.0 & $\mathrm{np}$ & 0.0 & nf & 0.0 & ne & 0.0 & ne & 17.0 .1 & ne & 98.0 \\
\hline 14 & Tpk & 0.0 & $\mathrm{np}$ & 0.0 & nf & 0.0 & ne & 0.0 & ne & 17.0 & ne & 193.0 \\
\hline 15 & Tpc un & 0.0 & pe & 0.0 & pe & 0.0 & pe & 0.0 & pe & 17.0 & pe & 206.0 \\
\hline 16 & Tpсpv3 & & np-td & 318.0 & nf & 140.0 & nf & 135.3 & nf & 69.7 & nf & 578.0 \\
\hline 17 & Tpcpv2 & & & 318.0 & $c-v$ & 140.0 & $c-w$ & 135.3 & $c-v$ & 69.7 & $\mathrm{c}-\mathrm{v}$ & 578.0 \\
\hline 18 & Tpcpvl & & & 323.0 & $w$ & 154.0 & $w$ & 151.8 & $\mathbf{w}$ & 79.2 & $w$ & 589.0 \\
\hline 19 & Tpbt4 & & & 338.0 & $d$ & 163.0 & d & 158.6 & d & 102.0 & d & 597.0 \\
\hline 20 & Tpy & & & 344.0 & $d$ & 170.0 & $d$ & 162.8 & $\mathrm{nf}$ & 106.4 & d & 600.0 \\
\hline 21 & Tpbt3 & & & 354.0 & d & 187.0 & $d$ & 162.8 & d & 156.0 & d & 600.0 \\
\hline 22 & Tpp & & & 375.0 & d & 215.0 & $d$ & 174.9 & d & 172.0 & $d$ & 621.0 \\
\hline 23 & Tpbi2 & & & 458.0 & d & 288.0 & d & 220.8 & d & 258.8 & d & 621.0 \\
\hline 24 & Tptrv3 & & & 477.0 & d & 321.0 & d & 244.7 & d & 284.3 & d & 643.0 \\
\hline 25 & Tpirv2 & & & 481.5 & $w$ & 327.0 & $\mathbf{w}$ & 257.4 & w & 292.7 & $\mathbf{w}$ & 653.0 \\
\hline 26 & Tptrvl & & & 485.0 & $\mathbf{w}$ & 330.0 & w & 259.8 & $w$ & 296.2 & $w$ & 654.0 \\
\hline 27 & Tpton & & & 488.9 & $v-c$ & 332.0 & $\mathrm{v}-\mathrm{c}$ & 263.2 & $v-c$ & 299.0 & $v-c$ & 658.0 \\
\hline 28 & TptrI & & & 660.5 & c & 517.0 & c & $\begin{array}{l}429.0 \\
\end{array}$ & c & 478.2 & $c$ & 774.0 \\
\hline 29 & Tppff & & & 700.0 & nf & 565.0 & nf & 465.5 & nf & $518.4 \mathrm{f}$ & nf & 810.0 \\
\hline 30 & Tptpul & & & 700.0 & & 565.0 & $c$ & 465.5 & c & $518.4 \mathrm{C}$ & $\mathrm{c}$ & 810.0 \\
\hline 31 & Tptpmn & & & & np-td & 770.0 & $c$ & 713.0 & $c$ & 740.0 c & c & 977.0 \\
\hline 32 & Tptpll & & & & & 901.5 & c & 810.0 & c & $877.6 \mathrm{C}$ & c & 1100.0 \\
\hline 33 & Tptpln & & & & & 1230.0 & $c$ & & $n p-t d$ & 1243.0 & c & 1178.0 \\
\hline 34 & Tptpv3 & & & 1 & & & np-td & & & $1414.8 \mathrm{c}$ & $\mathrm{c}-\mathrm{v}$ & 1178.0 \\
\hline 35 & Tptpv2 & & & & & & & & & 1457.0 v & $\mathrm{w}>1466.0 \mathrm{az}$ & 1178.0 \\
\hline 36 & Tptpvl & & & & & & & & & 1474.6 & $\begin{array}{ll}\mathbf{w} & \mathrm{az} \\
\end{array}$ & 1213.0 \\
\hline 37 & Tpbul & & & & & & & & & $1493.0 \mathrm{C}$ & $\mathrm{az}$ & 1253.0 \\
\hline 38 & Tac & & & & & & & & & $1498.0 \mathrm{C}$ & $\mathrm{az}$ & 1274.0 \\
\hline 39 & Tacbt & & & & & & & & & & np-td & \\
\hline 40 & Tсp4v & & & & & & 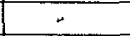 & & & & & \\
\hline 41 & $\operatorname{Tcp} 3 n 2 c$ & & & & & & & & & & & \\
\hline 42 & Tcp3m-d & & & & & & & & & & & \\
\hline 43 & Tcpl-3nlc & & & & & & & & & & & \\
\hline 44 & Tcpl-3nlv & & & & & & & & & & & \\
\hline 45 & Tcpbt & & & & & & & & & & & \\
\hline 46 & Tcbn2v & & & & & & & & & & & \\
\hline 47 & Tcbn2ć & & & & & & & & & ? & & \\
\hline 48 & Tcbm-d & & & & & & & & & & & \\
\hline 49 & Tcbnlc & & & & & & & & & & & \\
\hline 50 & Tcbnlv & & & & & & & & & & & \\
\hline 51 & Tcbbt & & & & & $\therefore$ & & & & & & \\
\hline 52 & $\operatorname{Tetn} 2 v$ & & & & & & & & & & & \\
\hline
\end{tabular}




\begin{tabular}{|c|c|c|c|c|c|c|c|c|c|c|c|c|}
\hline & $\mathbf{B A}$ & $\mathrm{BB}$ & $\mathrm{BC}$ & $\mathrm{BD}$ & $\overline{\mathrm{BE}}$ & $\mathrm{BF}$ & BG & $\mathrm{BH}$ & BI & BJ & $\mathrm{BK}$ & $\mathrm{BL}$ \\
\hline 53 & $\operatorname{Tctn} 2 \mathrm{c}$ & & & & & & & & & & & \\
\hline 54 & Tetm-d & & & & & & & & & & & \\
\hline 55 & Tetnlc & & & & & $i$ & & & & & & \\
\hline 56 & Tctnlv & & & & & & & & & & & \\
\hline 57 & Tctbt & & & & & & & & & & & \\
\hline 58 & Tund & & & & & & & & & & & \\
\hline 59 & $\mathbf{P z}$ & & & & & & & & & & & \\
\hline 60 & & & & & & & & & & & & \\
\hline 61 & $\mathrm{~V}-\mathrm{Z}$ & & np a & & np a & & $n p \quad a$ & & $\mathrm{np} \quad \mathrm{a}$ & 1466.0 & $\mathbf{a}$ & 1153.0 \\
\hline 62 & RHH & & np $\mathrm{g}$ & & np $g$ & 681.0 & $\mathrm{~g}$ & 620.0 & $\mathrm{~g}$ & $659.0 \mid \mathrm{g}$ & $g$ & 927.0 \\
\hline 63 & & & & 1 & & & & & & & & \\
\hline 64 & & & & & & & & & & & & \\
\hline 65 & $\mathrm{TD}$ & 330.0 & & 726.0 & & 1350.0 & & 1100.0 & & 1513.0 & & 1540.0 \\
\hline 66 & & & & & & & & & & & & \\
\hline 67 & & & & & 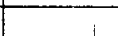 & & & & & & & \\
\hline 68 & & & & & & & & & & & & \\
\hline 69 & & & & & & & & & & & & \\
\hline 70 & & & & & & & & & & & & \\
\hline 71 & & & & & & & & & & & & \\
\hline 72 & & & & & & & & & & & & \\
\hline 73 & & & & . & & & & & & & & . \\
\hline 74 & & & & & & & & & & & & \\
\hline 75 & & & & & & & & & & & & \\
\hline 76 & & & & & & & & & & & & \\
\hline 77 & & & & & & 1 & & & & & & \\
\hline 78 & & & & & & & & & & & & \\
\hline 79 & & & 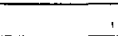 & & & & & & & & & \\
\hline 80 & & & & & & & & & & & & \\
\hline 81 & & & & & & & & & & & & \\
\hline 82 & & & & . & & & & & & & & \\
\hline 83 & & & & & & & & & & & & \\
\hline 84 & & & & & & & & & & & & \\
\hline 85 & & & & & & & & & & & & \\
\hline 86 & & & & & & & & & & & & \\
\hline 87 & & & & & & & & & & & & \\
\hline
\end{tabular}




\begin{tabular}{|c|c|c|c|c|c|c|c|c|c|c|c|c|c|c|}
\hline & $\mathrm{BM}$ & $\mathrm{BN}$ & BO & BP & $\mathrm{BQ}$ & $\overline{\mathrm{BR}}$ & $\mathrm{BS}$ & BT & $\mathrm{BU}$ & BV & BW & $\mathrm{BX}$ & BY & $B Z$ \\
\hline 1 & & & & & & & & & & & & & & \\
\hline 2 & & & & & & & & & & & & & & \\
\hline 3 & & & & & & & 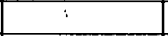 & & & & & & & \\
\hline 4 & & & & & & & & & & & & & & \\
\hline 5 & & & & & & & & & & & & & & \\
\hline 6 & & & & . & & & & & & & & & & \\
\hline 7 & & & & & & & & & & & & & & \\
\hline 8 & & & & & & & & & & & & & & \\
\hline 9 & Contact & & Contact & & Contact & & Contact & & Contact & & Contact & & Contact & \\
\hline$\frac{10}{11}$ & $\begin{array}{l}\text { tupe } \\
\text { nd }\end{array}$ & $\frac{U \mathrm{UE}-25 \mathrm{P} \# 1}{0.0}$ & ivpe & $\frac{\text { USW SD-7 }}{0.0}$ & tupe & \begin{tabular}{r|} 
USW SD-9 \\
0.0
\end{tabular} & $\begin{array}{l}\text { type } \\
\text { nc, dp }\end{array}$ & $\begin{array}{rr}\text { USW SD-12 } \\
0.0\end{array}$ & \begin{tabular}{|l|} 
type \\
nc
\end{tabular} & \begin{tabular}{|l|l} 
USW UZ-1 & \\
& 0.0
\end{tabular} & $\begin{array}{l}\text { type } \\
\text { nd }\end{array}$ & $\begin{array}{ll}\text { UE-2S UZ\#4 } & \\
& 0.0\end{array}$ & $\begin{array}{l}\text { type } \\
\text { nd }\end{array}$ & $\begin{array}{ll}\text { UE-25 UZ\#5 } & \\
& 0.0\end{array}$ \\
\hline 12 & $d$ & 0.0 & d & 50.1 & ne & 53.6 & d & 5.3 & ne & $\begin{array}{r}0.0 \\
\end{array}$ & ne & 0.0 & $d$ & 3.0 \\
\hline 13 & np -e & 127.0 & $n p-e$ & 50.1 & ne & 53.6 & nf & 5.3. & ne & $40.0 \mathrm{n}$ & ne & 39.0 & ne & 3.0 \\
\hline 14 & d & 127.0 & $n p-e$ & 50.1 & ne & 53.6 & nf & 5.3 .1 & ne & 40.0 & ne & 39.0 & ne & 3.0 \\
\hline 15 & pe & 127.0 & pe & 50.1 & pe & 53.6 & pe & $5.3 T_{1}$ & pe & $40.0 \mathrm{p}$ & pe & 39.0 & pe & 3.0 \\
\hline 16 & nf & 127.0 & $n p-e$ & 305.0 & nf & 57.2 & nf & 239.5 & nf & $40.0 \mathrm{n}$ & ne & 71.4 & nf & 89.0 \\
\hline 17 & $c-w$ & 127.0 & $n p-e$ & 305.0 & $\mathrm{c}-\mathrm{v}$ & 57.2 & $c-v$ & 239.5 & $\mathrm{c}-\mathrm{v}$ & $40.0 \mathrm{n}$ & ne & 71.4 & $\mathrm{c}-\mathrm{v}$ & 89.0 \\
\hline 18 & $\mathrm{w}$ & 127.0 & np-e & 316.0 & $\mathrm{w}$ & 76.5 & $\mathrm{w}$ & 256.0 & $\mathbf{w}$ & $40.0 \mathrm{r}$ & ne & 78.0 & $w$ & 100.0 \\
\hline 19 & d & 127.0 & $n p-e$ & 325.8 & d & 91.5 & d & 263.7 & d & 40.0 & ne & 99.0 & d & 118.0 \\
\hline 20 & nf & 127.0 & $n p-e$ & 330.6 & nf & 95.9 & d & 266.0 & nf & $40.0 \mathrm{n}$ & $n p-e$ & 106.0 & $d$ & 122.0 \\
\hline 21 & $d$ & 127.0 & $\mathrm{np}-\mathrm{e}$ & 330.6 & d & 140.8 & d & 266.0 & d & 78.0 & d & 151.5 & $d$ & 162.0 \\
\hline 22 & nf & 127.0 & np-e & 343.0 & $d$ & 155.5 & $d$ & 278.3 & d & 105.0 & $d$ & 173.9 & $d$ & 186.0 \\
\hline 23 & d & 127.0 & np-e & 356.0 & $d$ & 226.6 & d & 291.2 & d & 242.0 & d & 305.0 & d & 316.0 \\
\hline 24 & d & 140.0 & & 384.3 & d & 255.6 & $d$ & 314.1 & d & 272.0 & & 333.0 & d & 345.0 \\
\hline 25 & w & 145.0 & $\mathbf{w}$ & 384.3 & $\underline{w}$ & 266.7 & $\mathbf{w}$ & 320.8 & $w$ & 282.5 & $w$ & 343.0 & $w$ & 352.5 \\
\hline 26 & $w$ & 148.0 & $w$ & 386.3 & nf & 268.5 & w & 324.5 & $w$ & 284.0 & $w$ & 345.0 & $w$ & 354.5 \\
\hline 27 & $\mathrm{v}-\mathrm{c}$ & 150.0 & & 386.3 & $v-c$ & 272.2 & $v-c$ & 330.7 & $\mathrm{v}-\mathrm{c}$ & $288.0 \mathrm{~V}$ & $v-c$ & 346.0 & $v-c$ & 356.1 \\
\hline 28 & c & 228.0 & & 490.0 & c & 450.0 & $\mathrm{c}$ & 436.4 & c & $436.0 . \mathrm{C}$ & & & np-td & \\
\hline 29 & nf & 248.0 & & 490.0 & nf & 473.0 & nf & 470.2 & nf & 470.0 & nf & & & \\
\hline 30 & $\mathrm{c}$ & 248.0 & & 490.0 & $c$ & 473.0 & c & 470.2 & c & $470.0 \mathrm{c}$ & & & & \\
\hline 31 & $c$ & $493.0 \mathrm{c}$ & & 682.5 & c & 730.0 & c & 663.7 & c & $717.0 \mathrm{c}$ & & & & \\
\hline 32 & $c$ & 640.0 & & 803.3 & c & 845.8 & c & 786.9 & $c$ & $830.0 \mathrm{c}$ & & & & \\
\hline 33 & f & 958.0 & & 1020,0 & c & 1182.0 & $\mathrm{c}$ & 1065.5 & $c$ & $1145.0 \mathrm{c}$ & & & & \\
\hline 34 & $f$ & 1090.0 & faz & 1182.0 & $c-v$ & 1358.0 & $\mathrm{c}-\mathrm{v}$ & 1278.1 & $c-v$ & & np-td & & & \\
\hline 35 & $f>1153.0$ az & 1200.0 & $\mathrm{az}$ & 1285.0 & $w$ & 1418.4 & $w$ & 1308.0 & $\mathbf{w}$ & & & & & \\
\hline 36 & $\mathrm{az}$ & 1243.0 & $\mathrm{az}$ & 1308.0 & $w$ & 1425.7 & $w>1457.0 \mathrm{az}$ & 1337.5 & $\mathbf{w}$ & & & & & \\
\hline 37 & $3 z$ & 1270.0 .1 & nf & 1395.4 & d & 1464.1 & $\mathrm{az}$ & 1408.1 & d & & & & & \\
\hline 38 & $\mathrm{az}$ & 1270.0 & $a z$ & 1405.6 & $d$ & 1479.9 & az & 1411.5 & $\mathrm{~d}$ & & & & & \\
\hline 39 & np-td & 1390.0 & az & 1567.2 & $\mathrm{~d}>1562.0 \mathrm{az}$ & 1764.4 & $\mathrm{az}$ & 1599.5 & $d>1600$ az & & & & & \\
\hline 40 & & 1441.0 & az & 1621.5 & d & 1820.7 & az & 1648.4 & $\mathrm{az}$ & & & & & \\
\hline 41 & & 1468.0 & $v-c \quad(a z-c)$ & 1646.5 & $v-c(a z-c)$ & 1868.7 & $v-c$ & 1677.0 & $\mathrm{v}-\mathrm{c}$ & & & & & \\
\hline 42 & & 1535.0 & $\mathrm{w}, \mathrm{c}$ & 1765.0 & $c, w$ & 1938.5 & $c, w$ & 1787.0 & c, w & & & & & \\
\hline 43 & & 1630.0 & $\mathrm{w}, \mathrm{c}$ & 1832.0 & $c, w$ & 1991.4 & $c, w$ & 1842.0 & $c, w$ & & & & & \\
\hline 44 & & 1680.0 & $\mathrm{c}-\mathrm{v} \quad \mathrm{az}(\mathrm{c}-\mathrm{az})$ & 1872.0 & $c-v(c-a z)$ & 2015.8 & $\mathrm{c}-\mathrm{v}$ az (c-az) & $1865.0 \mathrm{c}$ & $c-v \quad a z(c-a z)$ & & & & & \\
\hline 45 & & 1790.0 & $\mathrm{az}$ & 2167.8 & $\mathrm{az}$ & & np-td & 2133.0 & $a z$ & & & & & \\
\hline 46 & & $1826.0 \mathrm{r}$ & $n p-e$ & 2183.9 & np-e & & & $2137.8 \mathrm{c}$ & az & & & & & \\
\hline 47. & & $1826.0 \mathrm{~s}$ & $n p-e$ & $\left.2183.9\right|_{t}$ & $n p-e$ & & & & np-td & & & & & \\
\hline 48 & & 1953.0 & $\mathrm{w}, \mathrm{c}$ & 2183.9 & $n p-e$ & & & & & & & & & \\
\hline 49 & & 2130.0 & $\mathrm{~N}, \mathrm{c}$ & $2450.0 . \mathrm{p}$ & $\mathrm{pe}$ & & & & & & & & & \\
\hline 50 & & $2162.0 \mid \mathrm{c}$ & $z-v \quad(c-a z)$ & $2478.0 \mathrm{c}$ & $\mathrm{c}-\mathrm{v}(\mathrm{c}-\mathrm{az})$ & & & & & & & & & \\
\hline 51 & & $2240.0 \mathrm{~d}$ & az & 2579.4 & d $\quad a z$ & & & & & & & & & \\
\hline 52 & & $2262.0] \mathrm{d}$ & $a z$ & 2598.0 & $\mathrm{az}$ & & & & & & & & & \\
\hline
\end{tabular}


0 .

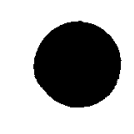

\begin{tabular}{|c|c|c|c|c|c|c|c|c|c|c|c|c|c|c|}
\hline & $\mathrm{BM}$ & $\mathrm{BN}$ & $\mathrm{BO}$ & $\mathrm{BP}$ & $\mathbf{B Q}$ & $\mathrm{BR}$ & BS & BT & BU & BV & BW & $\mathrm{BX}$ & BY & $\mathrm{BZ}$ \\
\hline 53 & & 2340.0 & $v-c \quad(a z-c)$ & 2611.8 & $v-c(a z-c)$ & & & & & & & & & \\
\hline 54 & & 2395.0 & $w, c$ & & np-Id & & & & & & & & & \\
\hline 55 & & 2595.0 & $w, c$ & & & & 1 & & & & & & & \\
\hline 56 & & 2616.0 & nf(?) & & & $\therefore$ & & & & & & & & \\
\hline 57 & & 2863.0 & $f, \mathrm{nf}(?)$ & & & & & & & & & & & \\
\hline 58 & & 2863.0 & $f, \mathbf{n f}(?)$ & & & $:$ & & & & & & & & \\
\hline 59 & & 4080.0 & $d$ or $f$ & & & & & & & & & & & \\
\hline 60 & & & & & & & & & & & & & & \\
\hline 61 & $\mathbf{a}$ & 1200.0 & $\mathbf{a}$ & 1562.0 & $a$ & 1457.0 & a & 1600.0 & a & & $\mathrm{np} \quad \mathrm{a}$ & & np a & \\
\hline 62 & $g$ & 453.0 & ni & 640.0 & $g$ & 628.0 & $g$ & 630.0 & $g$ & 585.0 & $\mathrm{~g}$ & & np $\mathrm{g}$ & \\
\hline 63 & & & & & & & & & & & & & & \\
\hline 64 & & & & & & & & & & & & & & \\
\hline 65 & & 5923.0 & & 2675.1 & & 2223.0 & & 2166.3 & & 1270.0 & & 411.0 & & 405.0 \\
\hline 66 & & & & & & & & & & & & & & \\
\hline 67 & & & & & & & & & & & & & & \\
\hline 68 & & & & & & & & & & & & & & \\
\hline 69 & & & & & & & & & & & & & & \\
\hline 70 & & & & & & & & & & & & & & \\
\hline 71 & & & & & & & & & & & & & & \\
\hline 72 & & & & & & & & & & & & & & \\
\hline 73 & & & & & & & & & & & & & & \\
\hline 74 & & & & & & & & & & & & & & \\
\hline 75 & & & & & & & & & & & & & & \\
\hline 76 & & & & & & & & & & & & & & \\
\hline 77 & & & & & & & & & & & & & & \\
\hline 78 & & & & & & & & & & & & & & \\
\hline 79 & & & & & & & & & & & & & & \\
\hline 80 & & & & & & & & & & & & & & \\
\hline 81 & & & & & & & & & & & & & & \\
\hline 82 & & & & & & & & & & & & & & \\
\hline 83 & & & & & & & & & & & & & & \\
\hline 84 & & & & & & & & & & & & & & \\
\hline 85 & & & & & & & & & & & & & & \\
\hline 86 & & & & & & & & & & & & & & \\
\hline 87 & & & & & & & & & & & & & & \\
\hline
\end{tabular}




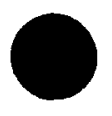

\begin{tabular}{|c|c|c|c|c|c|c|c|c|c|c|c|c|c|}
\hline & $\mathrm{CA}$ & $\mathrm{CB}$ & $\mathrm{CC}$ & $\mathrm{CD}$ & $\mathrm{CE}$ & $\mathrm{CF}$ & $\mathrm{CG}$ & $\mathrm{CH}$ & $\mathrm{CI}$ & CJ & CK & $\mathrm{CL}$ & $\mathrm{CM}$ \\
\hline \multicolumn{14}{|l|}{1} \\
\hline \multicolumn{14}{|c|}{$\frac{2}{3}$} \\
\hline \multirow{2}{*}{\multicolumn{14}{|c|}{\begin{tabular}{l|}
3 \\
4
\end{tabular}}} \\
\hline & & & & & & & & & & & & & \\
\hline \multicolumn{14}{|l|}{5} \\
\hline \multicolumn{14}{|c|}{\begin{tabular}{l|}
6 \\
7
\end{tabular}} \\
\hline \multirow{2}{*}{\multicolumn{14}{|c|}{$\begin{array}{l}7 \\
8 \\
\end{array}$}} \\
\hline & & & & & & & & & & & & & \\
\hline 9 & Contact & & Contact & & \begin{tabular}{|l|} 
Contact \\
\end{tabular} & & Contact & & Contact & & Contact & & Contact \\
\hline $\begin{array}{ll}10 \\
11\end{array}$ & type & $\frac{\text { USW UZ-6 }}{0.0}$ & $\frac{\text { tune }}{\text { nd }}$ & \begin{tabular}{|l|} 
USWUZ-7A \\
0.0
\end{tabular} & \begin{tabular}{|l|} 
tyne \\
nc
\end{tabular} & \begin{tabular}{|r|} 
USWUZ-14 \\
0.0
\end{tabular} & tope & $\begin{array}{|ll|}\text { UE-25 UZ\#16 } & \\
& 0.0\end{array}$ & type & USW UZ-N11 & type & USW UZ-N15 & 0 \\
\hline$\frac{11}{12}$ & nd & \begin{tabular}{|r|}
0.0 \\
0.0 \\
\end{tabular} & ne & $\begin{array}{r}38.5 \\
\end{array}$ & ne & $\begin{array}{r}39.7 \\
\end{array}$ & nd & 0.0 & $\mathrm{~d}$ & 0.0 & d & $\begin{array}{l}0.0 \\
0.0\end{array}$ & $\frac{0 \text { nd }}{0 \text { d }}$ \\
\hline 13 & ne & 0.0. & ne & 38.5 & ne & 39.7 & ne & 39.7 & & 1.7 & ne & & 3 ne \\
\hline 14 & ne & 0.0 & ne & 38.5 & ne & 39.7 & ne & 39.7 & ne & & ne & & 3 ne \\
\hline 15 & pe & 0.0 & pe & 38.5 & $\mathrm{pe}$ & 39.7 & $\mathrm{pe}$ & 39.7 & & 1.7 & pe & & 3 pe \\
\hline 16 & $n f$ & 383.0 & $\mathrm{c}-\mathrm{v}$ & 163.9 & nf & 39.7 & np-e & 140.8 & nf & 25.2 & nf & & np-td \\
\hline 17 & $c-v$ & 407.0 & $w$ & 163.9 & $\mathrm{c}-\mathrm{v}$ & 39.7 & np-e & 140.8 & $\mathrm{c}-\mathrm{v}$ & 25.2 & $\mathrm{c}-\mathrm{v}$ & & \\
\hline 18 & $w$ & 422.0 & $w$ & 184.0 & $w$ & 39.7 & np-e & 153.0 & $w$ & 29.7 & $w$ & & \\
\hline 19 & d & 432.5 & d & 197.7 & d & 39.7 & np-e & 160.7 & d & 46.4 & d & & \\
\hline 20 & d & 437.2 & nf & 203.6 & nf & 39.7 & np-e & 165.9 & & 60.6 & & & \\
\hline 21 & $d$ & 437.2 & d & 203.6 & d & 78.2 & d & 173.4 & & & np-td & & \\
\hline 22 & $d$ & 450.1 & d & 214.9 & d & 102.1 & $d$ & 188.8 & nf & & & & \\
\hline 23 & $d$ & 455.5 & $d$ & 218.6 & $d$ & 240.4 & $d$ & 188.8 & d & & & & \\
\hline 24 & d & 478.2 & d & 243.0 & d & 268.2 & d & 217.0 & $d$ & & & & \\
\hline 25 & $w$ & 483.0. & $w$ & 247.3 & $w$ & 280.9 & w & 228.1 & $w$ & & & & \\
\hline 26 & $w$ & 489.0 & $w$ & 248.6 & nf & 282.5 & $\mathbf{w}$ & 229.4 & $w$ & & & & \\
\hline 27 & $v-c$ & 490.0 & $\mathrm{v}-\mathrm{c}$ & 248.6 & $v-c$ & 286.0 & $v-c$ & 238.9 & $v-c$ & & & & \\
\hline 28 & $\mathrm{np-1d}$ & 575.0 & $c$ & 377.8 & nf & 430.0 & c & 357.8 & c & & & & \\
\hline 29 & & 610.0 .1 & nf & 377.8 & nf & 468.0 & nf & 371.0 & nf & & & & \\
\hline 30 & & 610.0 & $c$ & 377.8 & c $\quad($ fi $)$ & 468.0 & c & 371.0 & $c$ & & & & \\
\hline 31 & & 778.0 & c & 480.0 & $c$ & 715.0 & c & 545.0 & c & & & & \\
\hline 32 & & $917.0 \mathrm{~d}$ & c & 607.0 & c & 8828.0 & c & 669.0 & $c$ & & & & \\
\hline 33 & & $1190.0 \mathrm{c}$ & c & & np-td & 1138.0 & c & 935.0 & $\mathrm{c}$ & & & & \\
\hline 34 & & $1333.0 \mathrm{c}$ & $\mathrm{c}-\mathrm{v}$ & & & 1279.1 & $\mathrm{c}-\mathrm{v}$ & 1107.5 & $c-v$ & & & & \\
\hline 35 & & $1379.0 \mathrm{~V}$ & $w$ & & & 1358.0 & $w$ & 1165.2 & $\mathrm{az}$ & & & & \\
\hline 36 & & $1422.0 \mathrm{v}$ & $w$ & & $\therefore$ & 1383.0 & $\mathrm{w}>1395.5 \mathrm{az}$ & 1178.0 & $\mathrm{az}$ & & & & \\
\hline 37 & & 1450.0 & d & & & 1404.2 & az & 1190.0 & $\mathrm{az}$ & & & & \\
\hline 38 & & 1460.0 & d & & & 1420.2 & $\mathrm{az}$ & 1197.0 & $\mathrm{az}$ & & & & \\
\hline 39 & & $1547.2 \mathrm{c}$ & d & & & 1694.0 & az & 1455.4 & az & & & & \\
\hline 40 & & 1592.0 & d & & & 1750.2 & $\mathrm{az}$ & 1485.0 & $\mathrm{az}$ & & & & \\
\hline 41 & & 1614.0 & $\mathrm{v-c}$ & & & 1815.1 & $v-c$ & 1497.7 & $v-c$ & & & & \\
\hline 42 & & 1750.0 . & $c, w$ & & & 1850.0 & $c, w \quad$ (11) & 1571.0 & $c, w$ & & & & \\
\hline 43 & & 1802.0 & $c, w$ & & & 1893.0 & $c, w$ & 1638.0 & $c, w$ & & & & \\
\hline 44 & & 1829.0 & $c-v \quad a z(c-a z)$ & & & 1899.0 & $\mathrm{c}-\mathrm{v} \quad \mathrm{az}(\mathrm{c}-\mathrm{az})$ & 1669.2 & $c-v$ az $(c-a z)$ & & & & \\
\hline 45 & & & $n p-t d$ & & & 2046.6 & $\mathrm{az}$ & & np-td & & & & \\
\hline 46 & & . & & & & 2072.1 & $a z$ & & & & & & \\
\hline 47 & & & & & & & np-td & & & & & & \\
\hline 48 & & & & & & & & & & & & & \\
\hline 49 & & & & & & & & & & & & & \\
\hline 50 & & & & & & & & & & & & & \\
\hline 51 & & & & & & & & & & & & & \\
\hline 521 & & & & & & & & & & & & & \\
\hline
\end{tabular}



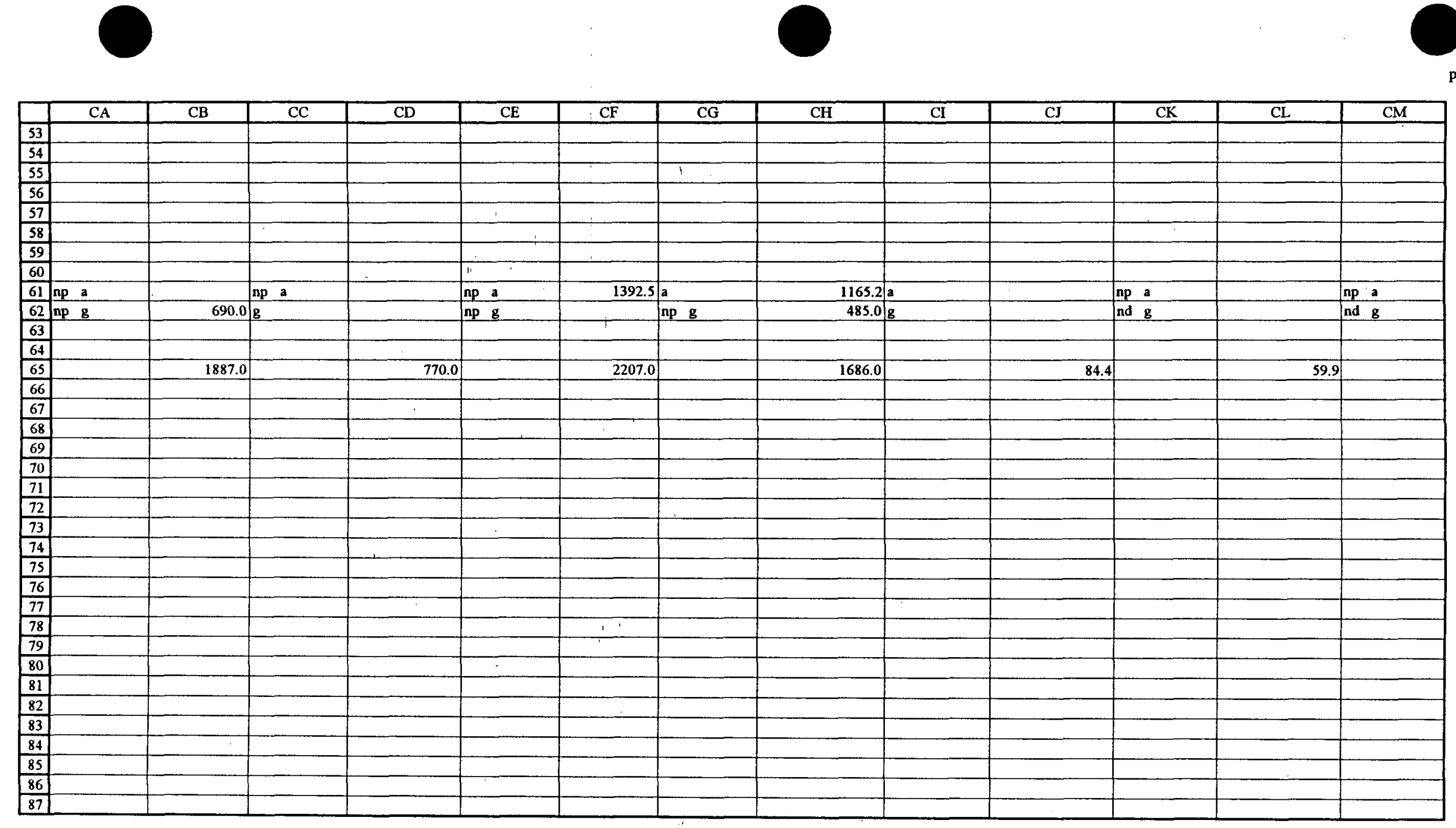


\section{○}

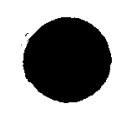

p. I- $/ 7$ of $<8$

\begin{tabular}{|c|c|c|c|c|c|c|c|c|c|c|c|c|c|}
\hline & $\mathrm{CN}$ & $\mathrm{CO}$ & $\mathrm{CP}$ & $\mathrm{CQ}$ & $\mathrm{CR}$ & $\mathrm{CS}$ & CT & $\mathrm{CU}$ & $\mathrm{CV}$ & $\mathrm{CW}$ & $\mathrm{CX}$ & CY & $\mathrm{CZ}$ \\
\hline 1 & & & . & & & & & & & & & & \\
\hline 2 & & & & & & & & & & & & & \\
\hline 3 & & & & & & $\because$ & & & & & & & \\
\hline 4 & & & & & & & & & & & & & \\
\hline 5 & & & & & & & & & & & & & \\
\hline 6 & & & & & & & & & & & & & \\
\hline 7 & & & & & & & & & & & & & \\
\hline 8 & & & & & & & & & & & & & - \\
\hline 9 & & Contact & & Contact & & Contact & & Contact & & Contact & & Contact & \\
\hline$\frac{10}{11}$ & USWUZ-N16 & tope & USWUZ-N17 & tope & USWUZ-N27 & one & USW UZ-N31 & type & USW UZ-N32 & type & USW UZ-N33 & type & USW UZ-N34 \\
\hline$\frac{11}{12}$ & 0.0 & d & \begin{tabular}{|r|}
0.0 \\
0.0 \\
\end{tabular} & did & \begin{tabular}{|r|}
0.0 \\
0.0 \\
\end{tabular} & ne & $\begin{array}{r}0.0 \\
15.0\end{array}$ & & 0.0 & ne & $\begin{array}{r}0.0 \\
13.1 \\
\end{array}$ & $d$ & 0.0 \\
\hline 13 & 4.2 & ne & 2.5 & ne & 0.0 & ne & 15.0 & ne & 0.0 & ne & 13.1 & ne & 50.1 \\
\hline 14 & 4.2 & ne & 2.5 & ne & 0.0 & ne & 15.0 & ne & 0.01 & ne & 13.1 & ne & 50.1 \\
\hline 15 & 4.2 & $\mathrm{pe}$ & 2.5 & pe & & pe & 15.0 & $\mathrm{pe}$ & 0.01 & pe & 13.1 & pe & 50.1 \\
\hline 16 & & np-td & & np-ld & & $n p-t d$ & 87.5 & nf & $96.0 \mathrm{~s}$ & nf & 13.1 & nf & 50.1 \\
\hline 17 & & & & & & & 87.5 & $\mathrm{c}-\mathrm{v}$ & 96.0 & $c-v$ & 13.1 & $c-v$ & 50.1 \\
\hline 18 & & & & & & & 102.7 & $w$ & 107.5 & & 22.6 & $\mathbf{w}$ & 50.1 \\
\hline 19 & & & & & & & 106.7 & & 113.1 & & 44.2 & $d$ & 50.1 \\
\hline 20 & & & & & & & 111.5 & nf & 118.0 & & 47.3 & d & 50.1 \\
\hline 21 & & & & & & & 111.5 & $d$ & 118.0 & & & np-td & \\
\hline 22 & & & & & & & 119.6 & $d$ & 130.0 & & & & \\
\hline 23 & & & & & & & 144.5 & $d$ & 158.5 & d & & & \\
\hline 24 & & & & & & & 168.2 & $\mathrm{~d}$ & 186.0 & & & & \\
\hline 25 & & & & & & & 179.9 & $\mathbf{w}$ & 198.1 & $w$ & & & \\
\hline 26 & & & & & & & 181.2 & $\mathrm{w}$ & 199.4 & & & & \\
\hline 27 & & & & & & & 185.5 & $v-c$ & 202.4 & & & & \\
\hline 28 & & & & & & & & np-td & & np-td & & & \\
\hline 29 & & & & & & & & & & & & & \\
\hline 30 & & & & & & & & & & & & & \\
\hline 31 & & & & & & & & & & & & & \\
\hline 32 & & & & & & & & & & & & & \\
\hline 33 & & & & & & & & & & & & & \\
\hline 34 & & & & & & & & & & & & & \\
\hline 35 & & & & & & & & & & & & & \\
\hline 36 & & & & & & & & & & & & & \\
\hline 37 & & & & & & & & & 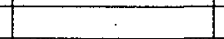 & & & & \\
\hline 38 & & & & & & & & & & & & & \\
\hline 39 & & & & & & & & & & & & & \\
\hline 40 & & & & & & & & & & & & & \\
\hline 41 & & & & & & & & & & & & & \\
\hline 42 & & & & & & & & & & & & & \\
\hline 43 & & & & & & & & & & & & & \\
\hline 44 & & & & & & & & & & & & & \\
\hline 45 & & & & & & & & & & & & & \\
\hline 46 & & & & & & & & & & & & & \\
\hline 47 & & & & & & & & & & & & & \\
\hline 48 & & & & & & & & & & & & & \\
\hline 49 & & & & & & & & & & & & & \\
\hline 50 & & & & & & & & & & & & & \\
\hline 51 & & & & & & & & & & & & & \\
\hline 52 & & & & & & & & & & & & & \\
\hline
\end{tabular}



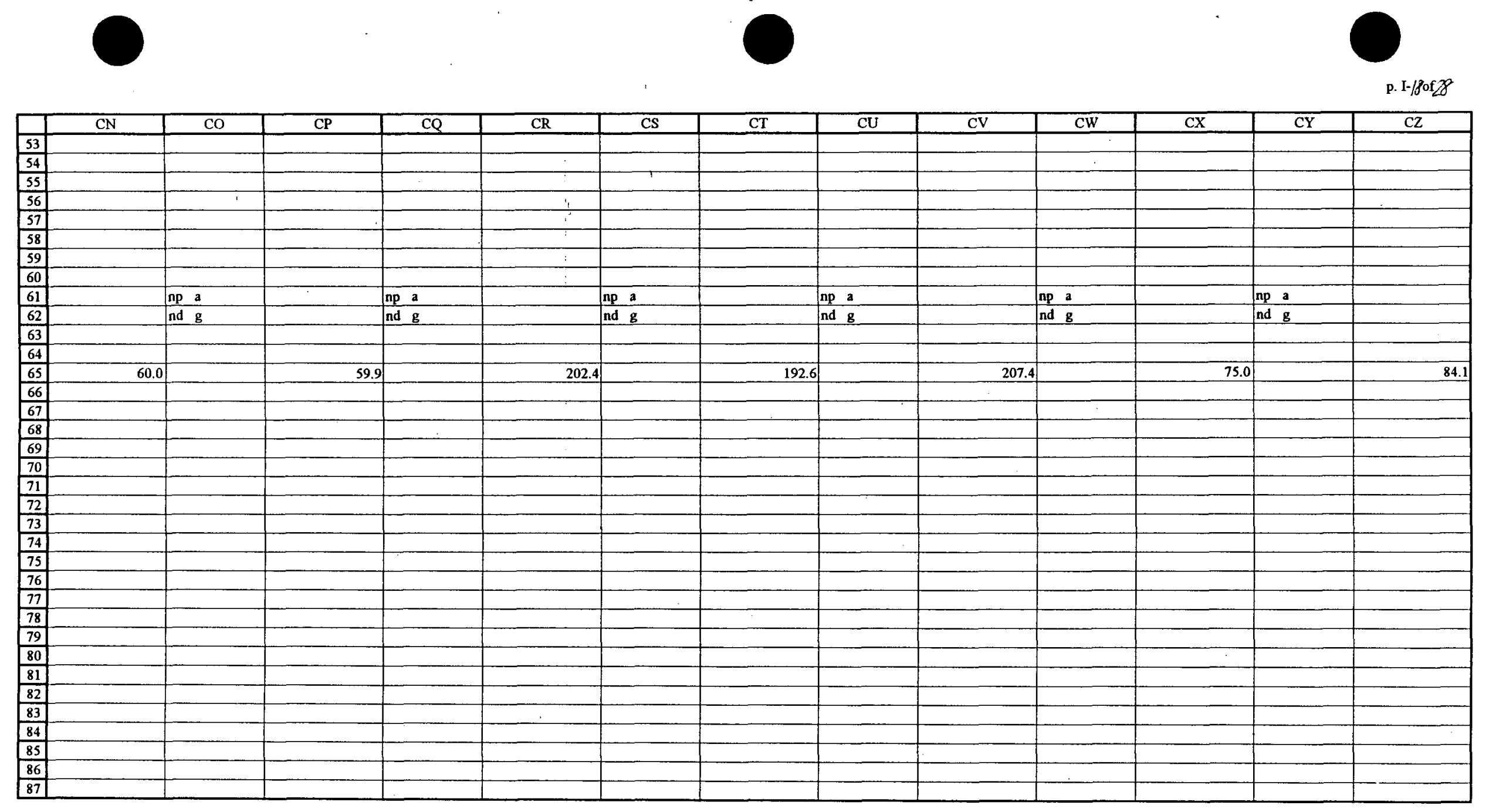


\begin{tabular}{|c|c|c|c|c|c|c|c|c|c|c|c|c|c|}
\hline & $\overline{D A}$ & $\overline{\mathrm{DB}}$ & $\overline{D C}$ & $\overline{D D}$ & $\overline{\mathrm{DE}}$ & DF & DG & $\overline{\mathrm{DH}}$ & $\mathrm{DI}$ & DJ & DK & $\overline{\mathrm{DL}}$ & DM \\
\hline 1 & & & & & & & & & & & & & \\
\hline 2 & & & & & & & & & & & & & \\
\hline 3 & & & & & & & & & & & & & \\
\hline 4 & & & & & & & & & & & & & \\
\hline 5 & & & & & & & & & & & & & \\
\hline 6 & & & & & & & & & & & & & \\
\hline 7 & & & & & & & & & & & & & \\
\hline 8 & & & & & & & & & & & & & \\
\hline 9 & Contact & & Contact & & Contact & & & & Contact & & Contact & & Contact \\
\hline $\begin{array}{l}10 \\
11 \\
\end{array}$ & $\begin{array}{l}\text { tope } \\
\text { nd }\end{array}$ & \begin{tabular}{|l|} 
USW UZ-N35 \\
0.0
\end{tabular} & tone & \begin{tabular}{r|} 
USW UZ-N36 \\
0.0
\end{tabular} & $\begin{array}{l}\text { inpe } \\
\text { ind }\end{array}$ & & \begin{tabular}{|l|} 
Symbol \\
NC
\end{tabular} & USW UZ-N37 & \begin{tabular}{|l|} 
type \\
nd
\end{tabular} & \begin{tabular}{|lll} 
USW UZ-N38 & \\
& 0.0 & 0 \\
\end{tabular} & type & $\begin{array}{|ll|}\text { USW UZ-N533 } & \\
& 0.0 \\
\end{array}$ & \begin{tabular}{|l} 
type \\
nd
\end{tabular} \\
\hline 12 & d & $\begin{array}{r}0.0 \\
\end{array}$ & $d$ & 0.0 & & & $\mathrm{Qa}$ & 0.0 & d & 0.00 & $d$ & 0.0 & d \\
\hline 13 & ne & 11.9 & ne & 0.9 & ne & & Tmr & 36.3 & ne & 17.9 . & ne & 2.1 & ne \\
\hline 14 & ne & 11.9 & ne & 0.9 & ne & & Tpk & 36.3 & ne & 17.9 & ne & 2.1 & ne \\
\hline 15 & pe & 11.9 & $p e$ & 0.9 & pe & & Tpc un & 36.3 & pe & 17.9 & pe & 2.1 & pe \\
\hline 16 & np-e & & np-td & & np-td & & Tpcpv3 & 109.4 & nf & 80.9 & $\mathrm{nf}$ & 150.6 & nf \\
\hline 17 & np-e & & & & & & Tpcpv2 & 109.4 & $c-v$ & 80.9 & $c-v$ & 150.6 & $c-v$ \\
\hline 18 & np-e & & & & & & Tpcpvl & 121.1 & $\mathrm{w}$ & & np-td & 159.2 & $w$ \\
\hline 19 & np-e & & & & & & Tpbt4 & 127.7 & $d$ & & & 173.4 & d \\
\hline 20 & $n p-e$ & & & & & & Tpy & 133.4 & nf & & & 175.2 & $d$ \\
\hline 21 & np-td & & & & & & Tpbt3 & 133.4 & d & & & 180.3 & d \\
\hline 22 & & & & & & & Tpp & 148.2 & d & & & 195.6 & nf \\
\hline 23 & & & & & & & Tpbt2 & 219.6 & $d$ & & & 195.6 & d \\
\hline 24 & & & & & & & Tptrv3 & 244.6 & $d$ & & & 220.8 & d \\
\hline 25 & & & & & & & Tptrv2 & 250.8 & $w$ & & & 227.7 & $w$ \\
\hline 26 & & & & & & & Tptrvl & 256.7 & $w$ & & & 230.1 & nf \\
\hline 27 & & & & & & & Tptrn & 258.0 & v-c & & & 230.1 & $v-c$ \\
\hline 28 & & & & & & & Tpert & & np-td & & & & np-td \\
\hline 29 & & & & & & & Tptf & & & & & & \\
\hline 30 & & & & & & & Tptpul & & & & & & \\
\hline 31 & & & & & & & Tptpmn & & & & & & \\
\hline 32 & & & & & & . & Tptpll & & & & & & \\
\hline 33 & & & & & $\%$ & & Tptpln & & & & & & \\
\hline 34 & & & & & + & & Tptpv3 & & & & & & \\
\hline 35 & & & & & & & Tptpv2 & & & & & & \\
\hline 36 & & & & & & & Tptpv1 & & & & & & \\
\hline 37 & & & & & & & Tpbt1 & & & & & & \\
\hline 38 & & & & & & & Tac & & & & & & \\
\hline 39 & & & & & & & Tacbt & & & & & & \\
\hline 40 & & & & & & & Tcp4v & & & & & & \\
\hline 41) & & & & & & & $T c p 3 n 2 c$ & & & & & & \\
\hline 42 & & & & & & & Tcp3m-d & & & & & & \\
\hline 43 & & & & & & & Tepl-3nlc & & & & & & \\
\hline 44 & & & & & & & Tcpl-3nlv & & & & & & \\
\hline 45 & & & & & & & Tepbt & & & & & & \\
\hline 46 & & & & & & & Tcbn2v & & & & & & \\
\hline 47 & & & & & & & Tcbn2c & & & & & & \\
\hline 48 & & & & & & & Tcbm-d & & & & & & \\
\hline 49 & & & & & & & Tcbnlc & & & & & & \\
\hline 50 & & & & & & & Tcbnlv & & & & & & \\
\hline 51 & & & & & & & Tcbbt & & & & & & \\
\hline 52 & & & & & & & $\operatorname{Tctn} 2 v$ & & & & & & \\
\hline
\end{tabular}



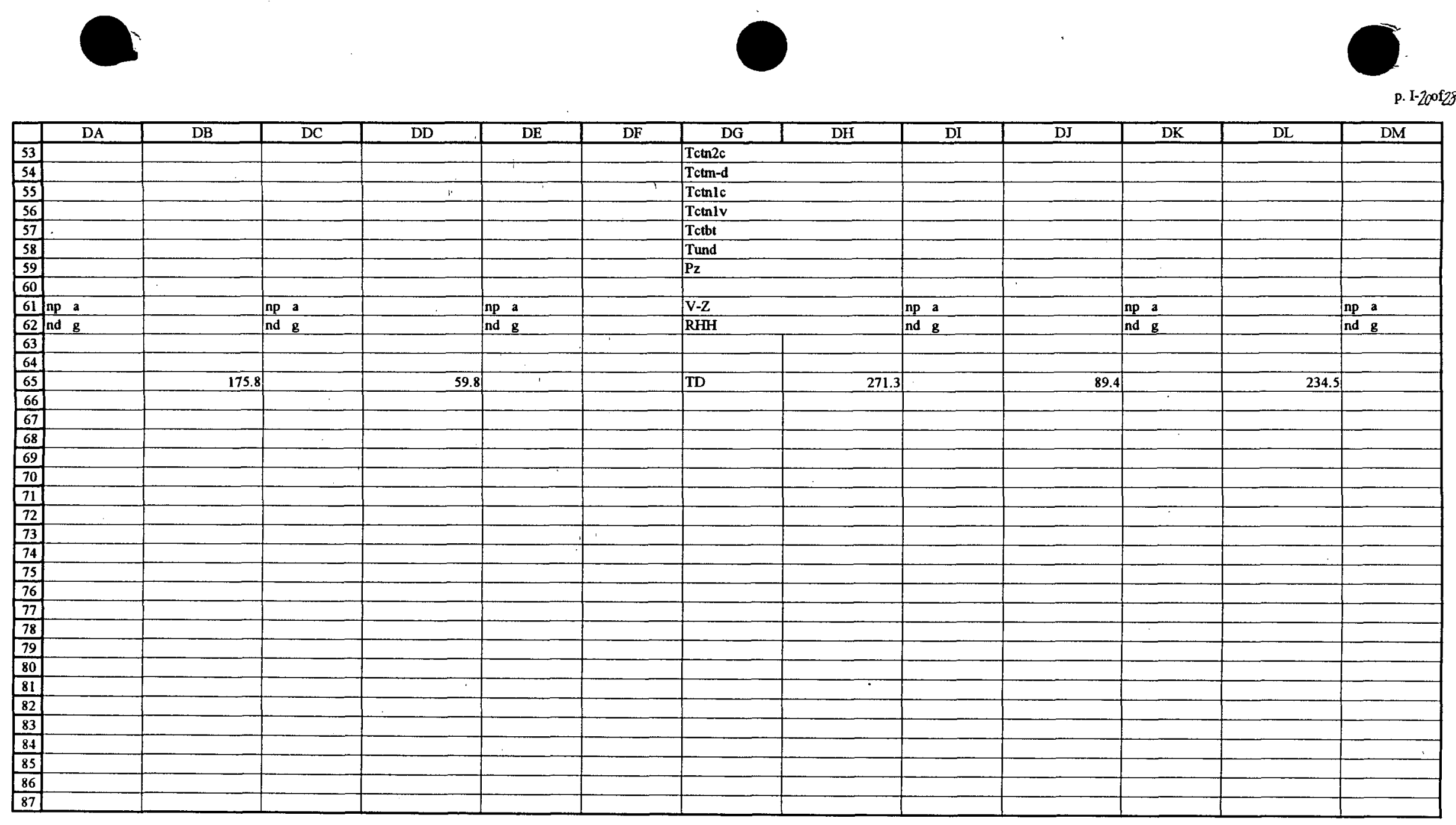

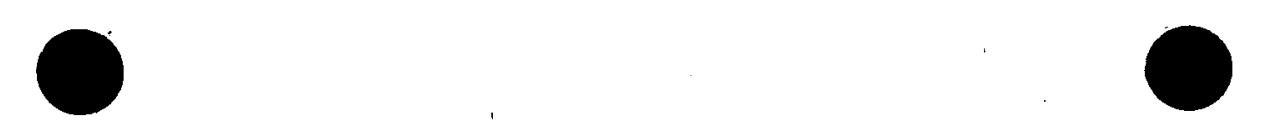

\begin{tabular}{|c|c|c|c|c|c|c|c|c|c|c|c|c|c|}
\hline & $\mathrm{DN}$ & DO & $\mathrm{DP}$ & $\mathrm{DQ}$ & $\mathrm{DR}$ & DS & DT & $\mathrm{DU}$ & DV & DW. & $\mathrm{DX}$ & DY & $\mathrm{DZ}$ \\
\hline 1 & & & & & & & & & & & & & \\
\hline 2 & & & & & & & & & & & & & \\
\hline 3 & & & & & & & & & & & & & \\
\hline 4 & & & & & & & & & & & & & \\
\hline 5 & & & & & & 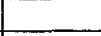 & & & & & & & 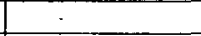 \\
\hline 6 & & & & & & & & & & & & & \\
\hline 7 & & & & & & & & & & & & & \\
\hline 8 & & & & & & & & & & & & & \\
\hline 9 & & Contact & & Contact & & Contact & & Contact & & Contact & & Contact & \\
\hline 10 & USWUZ-N54 & tope & USW UZ-N55. & tope & USW Uz-NS7 & wope & USW UZ-N58 & type & USW UZ-N59 & type & USW UZ-N61 & type & USW UZ-N62 \\
\hline 11 & 0.0 & Ind & 0.01 & nd & 0.0 & nc & $\mathbf{0 . 0}$ & nic & 0.0 & ne & 0.0 & 0 nc & 0.0 \\
\hline 12 & 0.0 & $d$ & 0.0 .1 & ne & 1.2 & d & 8.9 & $d$ & 13.1 & ne & & 8 ne & 1.0 \\
\hline 13 & 19.0 & ne & 0.0 & ne & 1.2 & ne & 22.1 & ne & 13.1 & ne & & $8 \mathrm{ne}$ & 1.0 \\
\hline 14 & 19.0 & ne & 0.0 & ne & 1.2 & ne & 22.1 & ne & 13.1 & ne & & 8 ne & 1.0 \\
\hline 15 & 19.0 & pe & 0.0 & pe & 1.2 & pe & 22.1 & pe & 13.1 & pe & 9.8 & $8 \mathrm{pe}$ & 1.0 \\
\hline 16 & 145.5 & nf & 177.0 & nf & & np-td & & np-td & & np-td & & np-td & \\
\hline 17 & 145.5 & $\mathrm{c}-\mathrm{v}$ & 177.0 & $\mathrm{c}-\mathrm{v}$ & & & & & & & & & \\
\hline 18 & 158.8 & $w$ & 190.0 & $w$ & & & & & & & & & \\
\hline 19 & 167.7 & d & 203.9 & d & & & & & & & & & \\
\hline 20 & 168.7 & $d$ & 207.9 & d & & & & & & & & & \\
\hline 21 & 175.5 & $d$ & 213.9 & $\mathrm{fb}$ & & & & & & & & & \\
\hline 22 & 191.4 & nf & 221.6 & nf & & & & & & & & & \\
\hline 23 & 191.4 & $d$ & 221.6 & 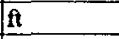 & & & & & & & & & \\
\hline 24 & 217.5 & $d$ & 233.4 & d & & & & & & & & & \\
\hline 25 & 227.7 & $w$ & 241.0 & $w$ & & & & & & & & & \\
\hline 26 & 232.6 & $\mathrm{nf}$ & 244.0 & $\mathbf{w}$ & & & & & & & & & \\
\hline 27 & 232.6 & $\mathrm{v}-\mathrm{c}$ & 246.6 & $v-c$ & & & & & & & & & \\
\hline 28 & & $n p-t d$ & & np-td & & & & & & & & & \\
\hline 29 & & & & & & & & & & & & & \\
\hline 30 & & & & & & & & & & & & & \\
\hline 31 & & & & & & & & & & & & & \\
\hline 32 & & & & & & & & & & & & & \\
\hline 33 & & & & & & & & & & & & & \\
\hline 34 & & & & & & & & & & & & & \\
\hline 35 & & & . & & & & & & & & & & \\
\hline 36 & & & & & & & & & & & & & \\
\hline 37 & & & & & & & & & & & & & \\
\hline 38 & & & & & & & & & & & & & \\
\hline 39 & & & & & & & & & & & & & \\
\hline 40 & & & & & & & & & & & & & \\
\hline 41 & & & & & & & & & & & & & \\
\hline 42 & & & & & & & & & & & & & \\
\hline 43 & & & & & & & & & & & & & \\
\hline 44 & & & & & & & & & & & & & \\
\hline 45 & & & & & & & & & & & & & \\
\hline 46 & & & & & & & & & & & & & \\
\hline 47 & & & & & & & & & & & & & \\
\hline 48 & & & & & & & & & & & & & \\
\hline 49 & & & & & & & & & & & & & \\
\hline S0 & & & & & & & & & & & & & \\
\hline 51 & & & & & & & & & & & & & \\
\hline 52 & & & & & & & & & & & & & \\
\hline
\end{tabular}



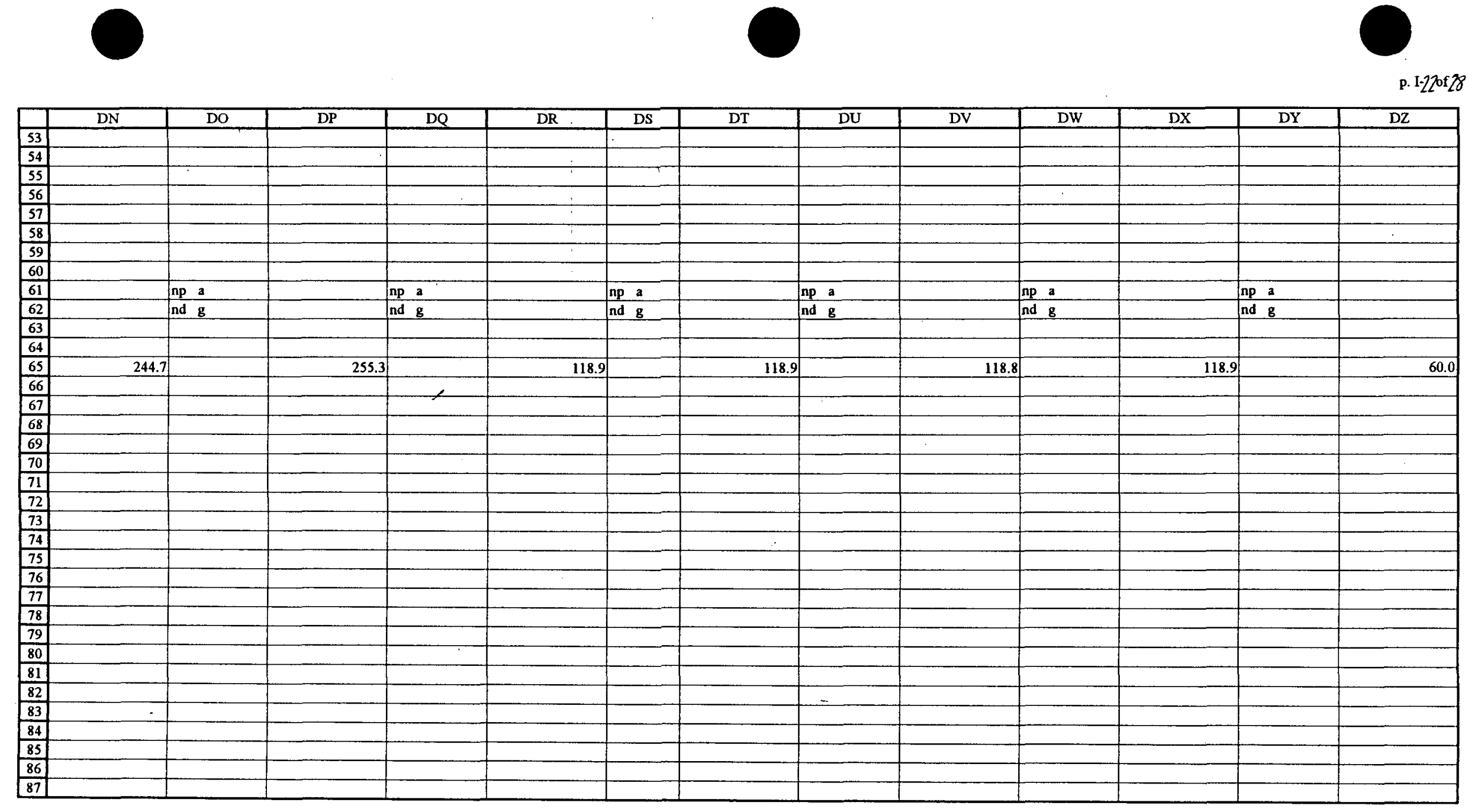


\begin{tabular}{|c|c|c|c|c|c|c|c|c|c|c|c|c|c|}
\hline & EA & $\mathrm{EB}$ & $\mathrm{EC}$ & ED & $E E$ & $\mathrm{EF}$ & $\mathbf{E G}$ & $\mathrm{EH}$ & EI & EJ & EK & EL & EM \\
\hline 1 & & & & & & & & & & & & & \\
\hline 2 & & & & & & & & & & & & & \\
\hline 3 & & & & & & & & & & & & & \\
\hline 4 & & & & & & & & & & & & & \\
\hline 5 & & & & & & & & & & & & & \\
\hline 6 & & & & & & & & & & & & & \\
\hline 7 & & & & & & & & & & 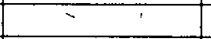 & & & \\
\hline 8 & & & & & & & & & & & & & \\
\hline 9 & Contact & & Contact & & Contact & & Contact & & Contact & & Contact & & Contact \\
\hline 10 & $\begin{array}{l}\text { twpe } \\
\text { nd }\end{array}$ & \begin{tabular}{|r|} 
USW UZ-N63 \\
0.0 \\
\end{tabular} & $\begin{array}{l}\text { Inpe } \\
\text { nd } \\
\end{array}$ & \begin{tabular}{|r|} 
USW UZ-N64 \\
0.0
\end{tabular} & the & USW WT-1 & $\begin{array}{l}\text { type } \\
\text { nd }\end{array}$ & \begin{tabular}{|r|} 
USW WT-2 \\
0.0 \\
\end{tabular} & $\begin{array}{l}\text { type } \\
\text { nd }\end{array}$ & UE-25 WT\#3 & $\begin{array}{l}\text { type } \\
\text { nd }\end{array}$ & $\begin{array}{lll}\text { UE-25 WT } \# 4 & 0.0 \\
& 0.0 \\
\end{array}$ & $\begin{array}{l}\text { type } \\
\text { ind }\end{array}$ \\
\hline 12 & ne & 0.0 & & 0.0 & $d$ & 0.0 & d & 0.0 & $d$ & 0.0 & $d$ & $0.0 \mathrm{~d}$ & $d$ \\
\hline 13 & ne & 4.4 & ne & & ne & $30.0 \mathrm{n}$ & ne, nf & 60.0 & ne & 11.0 & np-e & $51.0 \mathrm{n}$ & ne \\
\hline 14 & ne & 4.4 & ne & 1.8 & ne & $30.0 . \mathrm{r}$ & $\mathrm{ne}, \mathrm{nf}$ & 60.0 & ne & 11.0 & np-e & 51.0. & ne \\
\hline 15 & pe & 4.4 & pe & 1.8 & pe & 30.0 & pe & 60.0 & pe & 11.0 & np-e & & pe \\
\hline 16 & np-td & & np-td & & np-td & 395.0 & $\mathrm{c}-\mathrm{v}$ & 193.0 & $c-v$ & 11.0 & np-e & 261.0 .1 & $\mathrm{nf}$ \\
\hline 17 & & & & & & 410.0 & $w$ & 200.0 & $w$ & 11.0 & np-e & 261.0 & $\mathrm{c}-\mathrm{v}$ \\
\hline 18 & & & & & & $417.0 \mathrm{r}$ & $w$ & 215.0 & $w$ & 11.0 & np-e & 270.0 & paz?? \\
\hline 19 & & & & & & 431.0 & & 227.0 & & 11.0 & np-e & 281.0 & paz(?) \\
\hline 20 & & & & & & $435.0 \mathrm{r}$ & & 230.0 & $\mathrm{nf}$ & 11.0 & np-e & $293.0 \mathrm{r}$ & $n f$ \\
\hline 21 & & & & 1 & & 435.0 & $d$ & 230.0 & & 11.0 & np-e & $293.0 \mathrm{C}$ & paz(?) \\
\hline 22 & & & & & & $446.0 \mathrm{n}$ & & 247.0 & nf & 11.0 & np-e & 324.0 & $d$ \\
\hline 23 & & & & & & 446.0 & & 247.0 & & 11.0 & np-e & 419.0 & $\mathrm{azz}$ ? \\
\hline 24 & & & & & & $477.0 \mathrm{~d}$ & & 271.0 & & 11.0 & np-e & 444.0 & $d$ \\
\hline 25 & & & & & & 481.0 & $\mathrm{w}$ & 275.0 & $w$ & 11.0 & np-e & 448.0 & $\mathrm{w}$ \\
\hline 26 & & & & & & 484.0 & w & 280.0 & $w$ & 11.0 & np-e & 456.0 & $\mathrm{w}$ \\
\hline 27 & & & & & & $492.0 \mathrm{~V}$ & v-c & 285.0 & $\mathrm{v}-\mathrm{c}$ & 11.0 & $n p-e$ & $458.0 . \mathrm{V}$ & $v-c$ \\
\hline 28 & & & & & & $575.0 \mathrm{C}$ & & 380.0 & & $11.0 \mathrm{t}$ & np-e & 630.00 & $c$ \\
\hline 29 & & & & & & $593.0 \mathrm{n}$ & & 421.0 & & $11.0 \mathrm{t}$ & np-e & $630.0 \pi$ & nf \\
\hline 30 & & & & & & $593.0 \mathrm{c}$ & & 421.0 & & $11.0 \mathrm{n}$ & np-e & $660.0 \mathrm{c}$ & c \\
\hline 31 & & & & & & $733.0 \mathrm{c}$ & & 590.0 & & 11.0 & $n p-e$ & $727.0 \mathrm{c}$ & $\mathrm{c}$ \\
\hline 32 & & & & & & $888.0 \mathrm{c}$ & & 727.0 & & 11.0 & $\begin{array}{ll}\text { pe } & \mathrm{fb} \\
\end{array}$ & $785.0 \mid \mathrm{C}$ & c \\
\hline 33 & & & & & & $1187.0 \mathrm{c}$ & c & 1014.0 & & 35.01 & fit $\quad$ (12) & $1091.0 \mathrm{n}$ & nf \\
\hline 34 & & & & & & $1299.0 \mathrm{c}$ & $c-v$ & 1179.0 & $\mathrm{c}-\mathrm{v}$ & 189.0 & $\mathrm{faz}$ & $\begin{array}{ll}091.0 \mathrm{c} \\
\end{array}$ & faz \\
\hline 35 & & & & & & $1337.0 \mathrm{n}$ & az & 1223.0 & $w$ & 293.0 & az & 1122.0 & az \\
\hline 36 & & & & & & $1368.0 \mathrm{n}$ & az & 1264.0 & $w$ & 327.0 & az & $1141.0 \mathrm{H}$ & $a z$ \\
\hline 37 & & & & & & $1380.0 \mathrm{~d}$ & \begin{tabular}{|l}
$d \quad a z$ \\
\end{tabular} & 1315.0 & d & 351.0 & $\mathrm{az}$ & $1150.0 \mathrm{~d}$ & $\mathrm{az}$ \\
\hline 38 & & & & & & \begin{tabular}{ll|l}
1384.0 & $d$ \\
\end{tabular} & d fb az & 1319.0 & d 1435- Ist az & 358.0 & $\mathrm{az}$ & $1156.0 \mathrm{~d}$ & $\mathrm{az}$ \\
\hline 39 & & & & & & $1564.0 \mathrm{f}$ & & 1521.0 & & 461.0 & az & & np-td \\
\hline 40 & & & & & & $1564.0 \mathrm{f}$ & & 1594.0 & np-e & 512.0 & $\mathrm{az}$ & & \\
\hline 41 & & & & & & $1564.0 \mathrm{f}$ & & 1594.0 & $\mathrm{v}-\mathrm{c}$ & 554.0 & $v-c \quad(a z-c)$ & & \\
\hline 42 & & & & & & \begin{tabular}{l|l}
$1564.0 \mathrm{f}$ \\
\end{tabular} & & 1706.0 & $c, w$ & 660.0 & $\mathrm{c}, \mathbf{w}$ & & \\
\hline 43 & & & & & & $1564.0 \mathrm{f}$ & & 1776.0 & c, w & 704.0 & $\mathfrak{c}, \mathbf{w}$ & & \\
\hline 44 & & & & & & $1564.0 \mathrm{f}$ & $f$ & 1794.0 & $\mathrm{c}-\mathrm{v}$ az $(\mathrm{c}-\mathrm{az})$ & 710.0 & $\mathrm{c}-\mathrm{v} \quad$ az $(\mathrm{c}-\mathrm{az})$ & & \\
\hline 45 & & & & & & $1564.0 \mathrm{f}$ & $f$ & & np-td & 835.0 & $\mathrm{az}$ & & \\
\hline 46 & & & & & & $1564.0 \mathrm{f}$ & & & & 846.0 & d pe(?) az & & \\
\hline 47 & & & & & & \begin{tabular}{ll|l}
1564.0 & $\mathrm{f}$ \\
\end{tabular} & & & & 850.0 & $v-c \quad(a z-c)$ & & \\
\hline 48 & & & & & & $1564.0 \mathrm{f}$ & & 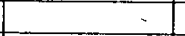 & & $909.0 \mathrm{c}$ & $c, w$ & & \\
\hline 49 & & & & & & & np-td. & & & & np-td & & \\
\hline 50 & & & & & & & & & & & & & \\
\hline 51 & & & & & & & & & & & & & \\
\hline \begin{tabular}{l|l}
52 & \\
\end{tabular} & & & & & & & & & & & & & \\
\hline
\end{tabular}




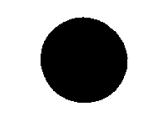

\section{0}

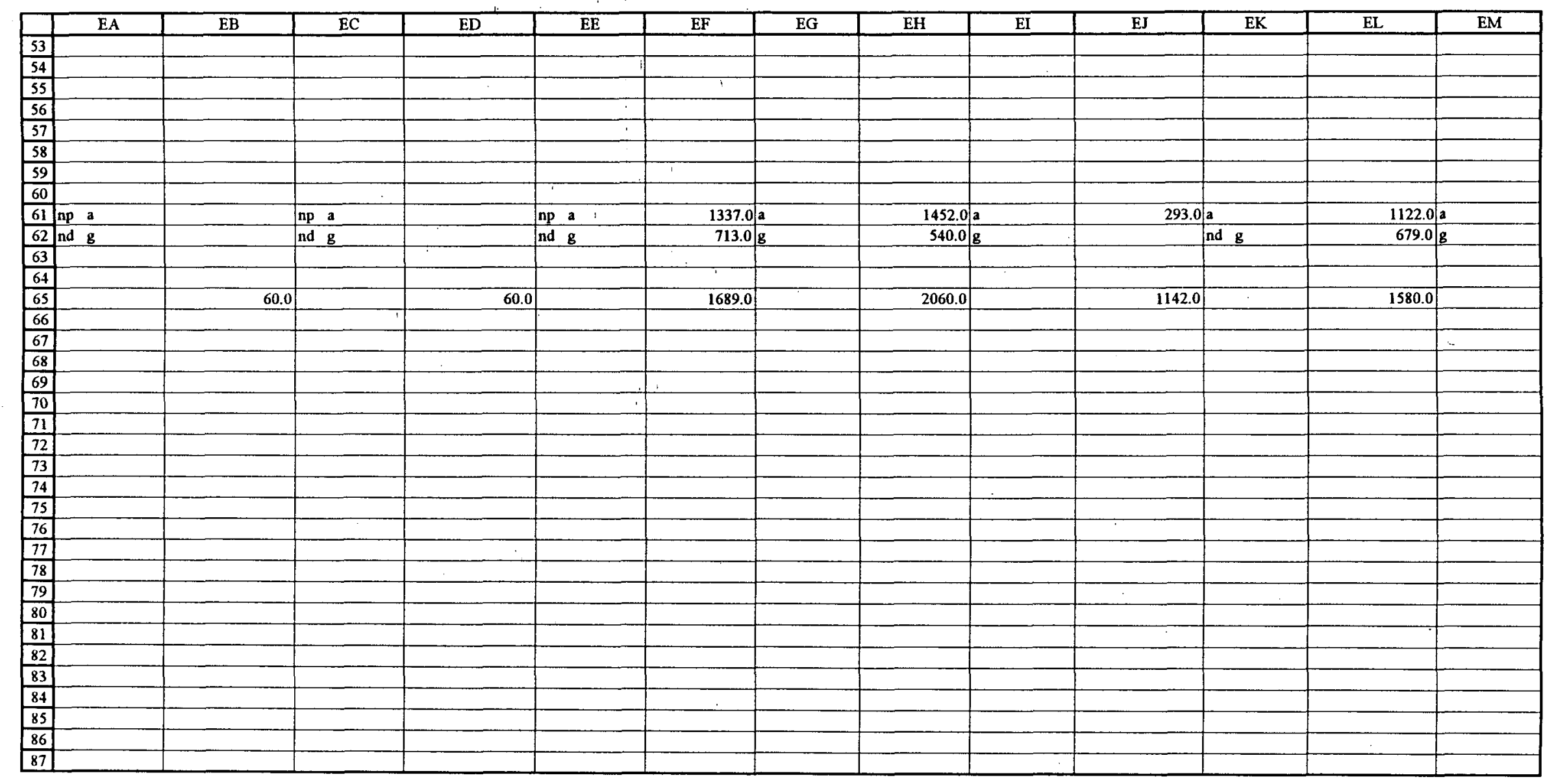




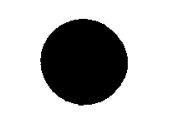

\begin{tabular}{|c|c|c|c|c|c|c|c|c|c|c|c|c|c|}
\hline & EN & EO & EP & $\mathbf{E Q}$ & ER & ES & ET & EU & EV & EW & EX & EY & EZ \\
\hline 1 & & & & & & & & & & & & & \\
\hline 2 & & & & & & & & & & & & & \\
\hline 3 & & & & & & & & & & & & & \\
\hline 4 & & & & & & & & & & & & & \\
\hline 5 & & & & & & & & & & & & & \\
\hline 6 & & & & & & & & & & & & & \\
\hline 7 & & & & & & & & & & & & & \\
\hline 8 & & & & & & & & & & & & & \\
\hline 9 & & Contact & & Contact & & Contact & & Contact & & Contact & & Contact & \\
\hline$\frac{10}{11}$ & \begin{tabular}{|c|}
$\mathrm{UE}-25 \mathrm{WT} \# 6$ \\
0.0
\end{tabular} & upe & $\frac{\mid \text { USW WT-7 }}{0.0}$ & ope & \begin{tabular}{|c|} 
USW WT-10 \\
0.0
\end{tabular} & ope & UE-25WJ\#11 & $\begin{array}{l}\text { type } \\
\text { nd }\end{array}$ & UE-25 WT\#12 & $\begin{array}{l}\text { type } \\
\text { nd }\end{array}$ & UE-25 WT\#13 & $\begin{array}{l}\text { type } \\
\text { nd }\end{array}$ & $\begin{array}{ll}\text { UE-25 WT\#14 } & \\
& 0.0\end{array}$ \\
\hline 12 & 0.0 & & 0.0 & & \begin{tabular}{|r|}
0.0 \\
\end{tabular} & $d$ & 0.0 & d & 0.0 & d & 0.0 & $d$ & 0.0 \\
\hline 13 & 170.0 & ne & 40.0 & & 60.0 & np-e & $40.0 \mathrm{n}$ & $\mathrm{ne}, \mathrm{nf}(?)$ & 60.0 & ne & $220.0 \mid \mathrm{r}$ & ne & 107.0 \\
\hline 14 & $170.0 / \mathrm{r}$ & ne & 40.0 & & 627.0 & nd & 40.0 & ne, nf & 60.0 & ne & 220.0 & ne & 107.0 \\
\hline 15 & 170.0 & $n p-e$ & 40.0 & $\mathrm{pe}$ & 627.0 & $d$ & 40.0 & pe & 60.0 & pe & 220.0 & pe & 107.0 \\
\hline 16 & 170.0 & np-e & 344.0 & $n f$ & 863.0 & $c-v$ & 239.0 & $c-v$ & 297.0 & $c-v$ & $416.0 . \mathrm{x}$ & nf & 107.0 \\
\hline 17 & 170.0 & np-e & 344.0 & $c-v$ & 872.0 & $w$ & $243.5 \mathrm{v}$ & $w$ & 300.0 & $w$ & $416.0 \mathrm{c}$ & $c-v$ & 107.0 \\
\hline 18 & 170.0 & np-e & 355.0 & $w$ & 880.0 & $w$ & 263.0 & $w$ & 306.0 & $w$ & 427.0 & $w$ & 107.0 \\
\hline 19 & 170.0 & np-e & 369.5 & $\mathrm{~d}$ & 887.0 & $d$ & 271.0 & d & 319.0 & $d$ & 440.0 & d & 107.0 \\
\hline 20 & 170.0 & np-e & 372.0 & & 894.0 & nf & 272.0 & nf & 323.0 & nf & 450.0 & nf & 107.0 \\
\hline 21 & 170.0 & $n p-\varepsilon$ & 374.5 & d & 894.0 & $d$ & 272.0 & d & 323.0 & $d$ & 450.0 & $d$ & 107.0 \\
\hline 22 & $170.0]_{\mathrm{I}}$ & np-e & 391.0 & nf & 924.0 & nf & 287.0 & nf & 339.0 & nf & 460.0 & d & 107.0 \\
\hline 23 & $170.0 \mathrm{x}$ & $n p-e$ & 391.0 & $d$ & 924.0 & d & 287.0 & d & 339.0 & $d$ & 469.0 & $d$ & 107.0 \\
\hline 24 & $170.0 \mathrm{~s}$ & np-e & 415.0 & & 954.0 & d & 307.0 & $d$ & 362.0 & d & 490.0 & d & 107.0 \\
\hline 25 & $170.0 \mathrm{~s}$ & np-e & 426.0 & $w$ & 960.5 & $w$ & 313.0 & $w$ & 365.0 & $w$ & 497.0 & w & 122.0 \\
\hline 26 & 170.0 .1 & np-e & 432.0 & $\mathrm{w}$ & 967.0 & $w$ & 317.0 & $w$ & 369.0 & $\mathbf{w}$ & $498.0 \mathrm{O}$ & $\mathbf{w}$ & 124.0 \\
\hline 27 & 170.0 & np-e & 435.0 & $v-c$ & 971.0 & $v-c$ & 324.0 & $v-c$ & 374.0 & $v-c$ & 500.0 & $v-c$ & 128.0 \\
\hline 28 & 170.0 & $n p-e$ & 515.0 & $c$ & 1035.0 & c & 430.0 .0 & $c$ & 478.0 & nf & 612.0 & $c$ & 247.0 \\
\hline 29 & 170.0 & pe & 546.0 & nf & 1049.0 & nf & 430.0 & nf & 478.0 & nf & 630.0 & nf & 275.0 \\
\hline 30 & 250.0 & nf & 546.0 & c & 1049.0 & c & 430.0 & & 478.0 & c & 630.0 & $c$ & 275.0 \\
\hline 31 & $\left.250.0\right|_{1}$ & nf & 706.0 & $\mathfrak{c}$ & 1250.0 & c & 661.0 & $c$ & 680.0 & $c$ & 755.0 & $c$ & 446.0 \\
\hline 32 & 250.0 & $\mathrm{~d}, \mathrm{c}$ & 959.0 & c & & np-td & $782.0 \mathrm{~g}$ & $c$ & 760.0 & c & 868.0 & c & 534.0 \\
\hline 33 & $303.0,1$ & nf & 1091.0 & c & & & 875.0 & $\mathrm{c}$ & \begin{tabular}{ll|l}
890.0 & \\
\end{tabular} & c & 1103.0 & & 830.0 \\
\hline 34 & 303.0 & faz & 1287.0 & $\mathrm{faz}$ & & & $1058.0 \mathrm{c}$ & $c-v$ & 1151.0 & $\mathrm{c}-\mathrm{v}$ & & np-td & 1024.0 \\
\hline 35 & 337.0 & $\mathrm{az}$ & 1351.0 & $a z$ & & & 1146.0 & paz & 1215.0 & $\mathbf{w}$ & & & 1117.0 \\
\hline 36 & 352.0 & az & 1360.0 & $a z$ & & & $1186.0 \mathrm{v}$ & $w$ & 1250.0 & $\mathbf{w}$ & & & 1137.0 \\
\hline 37 & 369.0 & $\mathrm{az}$ & 1433.0 & $\mathrm{az}$ & & & 1198.0 & d & $1259.0 \mathrm{C}$ & $d$ & & & 1157.0 \\
\hline 38 & 383.0 & az & 1438.0 & $\mathrm{az}$ & & & 1208.0 & $\mathrm{~d}>1271.0 \mathrm{az}$ & 1276.0 & $d$ & & & 12100 \\
\hline 39 & & np-td & 1510.0 & $\mathrm{az}$ & & & & np-td & & np-td & & & \\
\hline 40 & & & 1571.0 & $a z$ & & & & & & & & & \\
\hline 41 & & & 1598.0 & $\begin{array}{ll}\mathrm{v}-\mathrm{c} & (\mathrm{az}-\mathrm{c}) \\
\end{array}$ & & & & & & & & & \\
\hline+2 & & & & np-ld & & & & & & & & & \\
\hline 43 & & & & & & & & & & & & & \\
\hline 44 & & & & & & & & & & & & & \\
\hline 45 & & & & & & & & & & & & & \\
\hline 46 & & & $\therefore$ & & & & & & & & & & \\
\hline 47 & & & & & & & & & & & & & \\
\hline 48 & & & & & & & & & & & & & \\
\hline 49 & & & & & & & & & & & & & \\
\hline \begin{tabular}{l|l}
50 \\
51
\end{tabular} & & & & & & & & & & & & & \\
\hline$\frac{51}{52}$ & & & & & & & & & & & & & \\
\hline
\end{tabular}



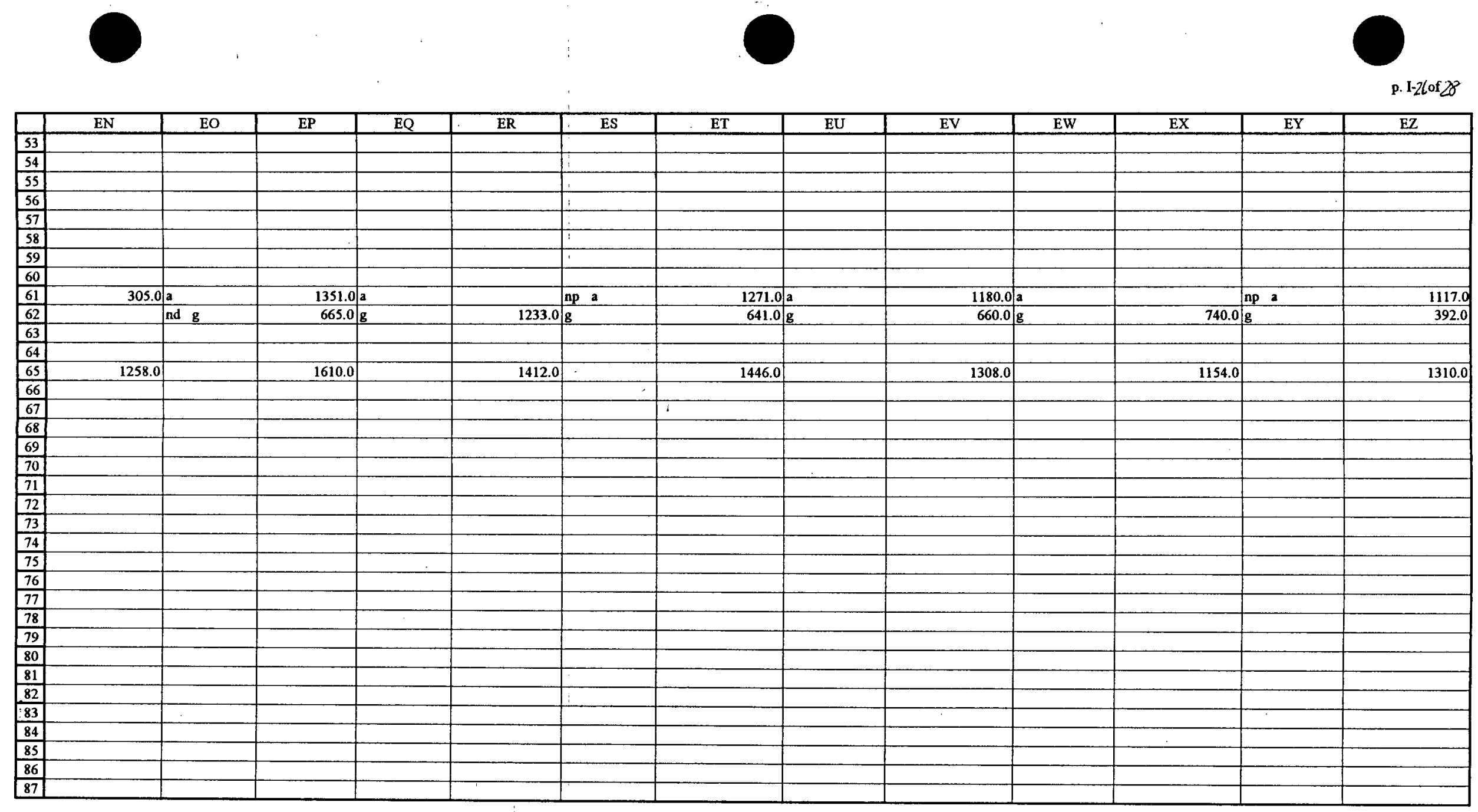


\begin{tabular}{|c|c|c|c|c|c|c|c|c|c|}
\hline & $\mathrm{FA}$ & $\mathrm{FB}$ & $\mathrm{FC}$ & FD & $\mathrm{FE}$ & $\mathrm{FF}$ & FG & $\mathrm{FH}$ & FI \\
\hline 1 & & & & & & & & & \\
\hline 2 & & & & & & & & & \\
\hline 3 & & & & & & & & & \\
\hline 4 & & & & & & & & & \\
\hline 5 & & & & & & & & & \\
\hline 6 & & & & & & & & & \\
\hline 7 & & & & & & & & & \\
\hline 8 & & & & & & & & & \\
\hline 9 & Contact & & Contact & & Contact & & \begin{tabular}{|l|} 
Contact \\
\end{tabular} & & Contact \\
\hline \begin{tabular}{|l|}
10 \\
11 \\
\end{tabular} & tope & $\begin{array}{r}0.0 \\
\text { UE-25 WTH15 } \\
0.0\end{array}$ & $\begin{array}{l}\text { tope } \\
\text { nd }\end{array}$ & \begin{tabular}{|l|l|} 
UE-25 WTH16 \\
0.0
\end{tabular} & Inpe & \begin{tabular}{ll|} 
UE-25 WTH17 & \\
& 0.0
\end{tabular} & $\begin{array}{l}\text { type } \\
\text { nd }\end{array}$ & \begin{tabular}{ll|} 
UE-25 WT\#18 & \\
& 0.0
\end{tabular} & $\begin{array}{l}\text { type } \\
\text { nd }\end{array}$ \\
\hline 12 & $d$ & 0.0 & d & 0.0 & & 0.0 & d & 0.0 & ne \\
\hline 13 & ne & 210.0 & np-e & 137.0 & $n p-c$ & 30.0 & $n p-c$ & 0.0 & ne \\
\hline 14 & ne & 210.0 & np-e & 137.0 & np-e & 30.0 & np-e & 0.0 & ne \\
\hline 15 & np-e & 210.0 & pe & 137.0 & pe & 30.0 & pe & 0.0 & $\mathrm{pe}$ \\
\hline 16 & npp-e & 332.0 & nf & 368.0 & nf & 188.0 & nf & 314.0 & nf \\
\hline 17 & $n p-e$ & 332.0 & $c-v$ & 368.0 & $c-v$ & 188.0 & $\mathrm{c}-\mathrm{v}$ & 314.0 & $c-v$ \\
\hline 18 & np-e & 334.0 & $\mathbf{w}$ & 375.0 & $w$ & 194.0 & $\mathbf{w}$ & 332.0 & $w$ \\
\hline 19 & $n p-e$ & 349.0 & d & 386.0 & $d$ & 197.0 & & 340.0 & d \\
\hline 20 & np-e & 356.0 & nf & 395.0 & $\mathrm{nf}$ & 203.0 & nf & 353.0 & $d$ \\
\hline 21 & $n p-e$ & 356.0 & d & 395.0 & $d$ & 203.0 & $d$ & 423.0 & d \\
\hline 22 & np-e & 372.0 & $f$ & 462.0 & $d$ & 217.0 & nf & 497.0 & d \\
\hline 23 & np-e & 413.0 & d & 558.0 & d & 217.0 & $d$ & 651.0 & $d$ \\
\hline 24 & np-e & 436.0 & d & 580.0 & $d$ & 242.0 & d & 692.0 & d \\
\hline 25 & $w$ & 440.0 & $w$ & 588.0 & $w$ & 245.0 & $w$ & 698.0 & $w$ \\
\hline 26 & $w$ & 442.0 & $w$ & 594.0 & \begin{tabular}{|ll}
$\boldsymbol{w}$ & 1
\end{tabular} & 248.0 & $\mathbf{w}$ & 701.0 & 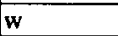 \\
\hline 27 & $v-c$ & 444.0 & $\mathrm{v}-\mathrm{c}$ & 596.0 & $v-c$ & 251.0 & $v-c$ & 702.0 & $\mathrm{v}-\mathrm{c}$ \\
\hline 28 & $c$ & 608.0 & $c$ & 818.0 & c & 312.0 & c & 879.0 & $c$ \\
\hline 29 & nf & 641.0 & nf & 830.0 & nf & 336.0 & nf & 900.0 & nf \\
\hline 30 & $c$ & 641.0 & & 830.0 & & 336.0 & c & 900.0 & $c$ \\
\hline 31 & $c$ & 852.0 & c & 830.0 & nf & 472.0 & $c$ & 1078.0 & c \\
\hline 32 & $c$ & 919.0 & $c$ & 830.0 & $\mathrm{nf}$ & 535.0 & c & 1170.0 & c \\
\hline 33 & $c$ & 1260.0 & $c$ & 1013.0 & & 668.0 & c & 1501.0 & nf \\
\hline 34 & $c-v$ & & $n p-t d$ & 1013.0 & $c-v$ & 874.0 & faz & 1501.0 & $\mathrm{c}-\mathrm{v}$ \\
\hline 35 & $\mathrm{az}$ & & & 1050.0 & $a z$ & 959.0 & $\mathrm{az}$ & 1564.0 & $\mathrm{az}$ \\
\hline 36 & az & & & 1057.0 & $\mathrm{az}$ & 989.0 & $\mathrm{az}$ & 1592.0 & $\mathbf{w}$ \\
\hline 37 & $\mathrm{az}$ & & & 1068.0 & nf & 998.0 & $\mathrm{az}$ & 1620.0 & nf \\
\hline 38 & az & & & 1068.0 & az & 998.0 & $\mathrm{az}$ & 1620.0 & $\mathrm{az}$ \\
\hline 39 & np-td & & & & np-td & 1184.0 & $a z$ & & np-td \\
\hline 40 & & & & & & 1271.0 & $\mathrm{az}$ & & \\
\hline 41 & & & & & & 1313.0 & $\begin{array}{ll}v-c & (a z-c) \\
\end{array}$ & & \\
\hline 42 & & & & . & & 1318.0 & $c, w$ & & \\
\hline 43 & & & & & & & np-td & & \\
\hline 44 & & & & & & & & & \\
\hline 45 & & & & & & & & & \\
\hline 46 & & & & & & & & & \\
\hline 47 & & & & & & & & & \\
\hline 48 & & & & & 1 & & & & \\
\hline 49 & & & & & & & & & \\
\hline 50 & & & & & & & & & \\
\hline 51 & & & & & & & & & \\
\hline 52 & & & & & & & & & \\
\hline
\end{tabular}




\section{$\bullet$}
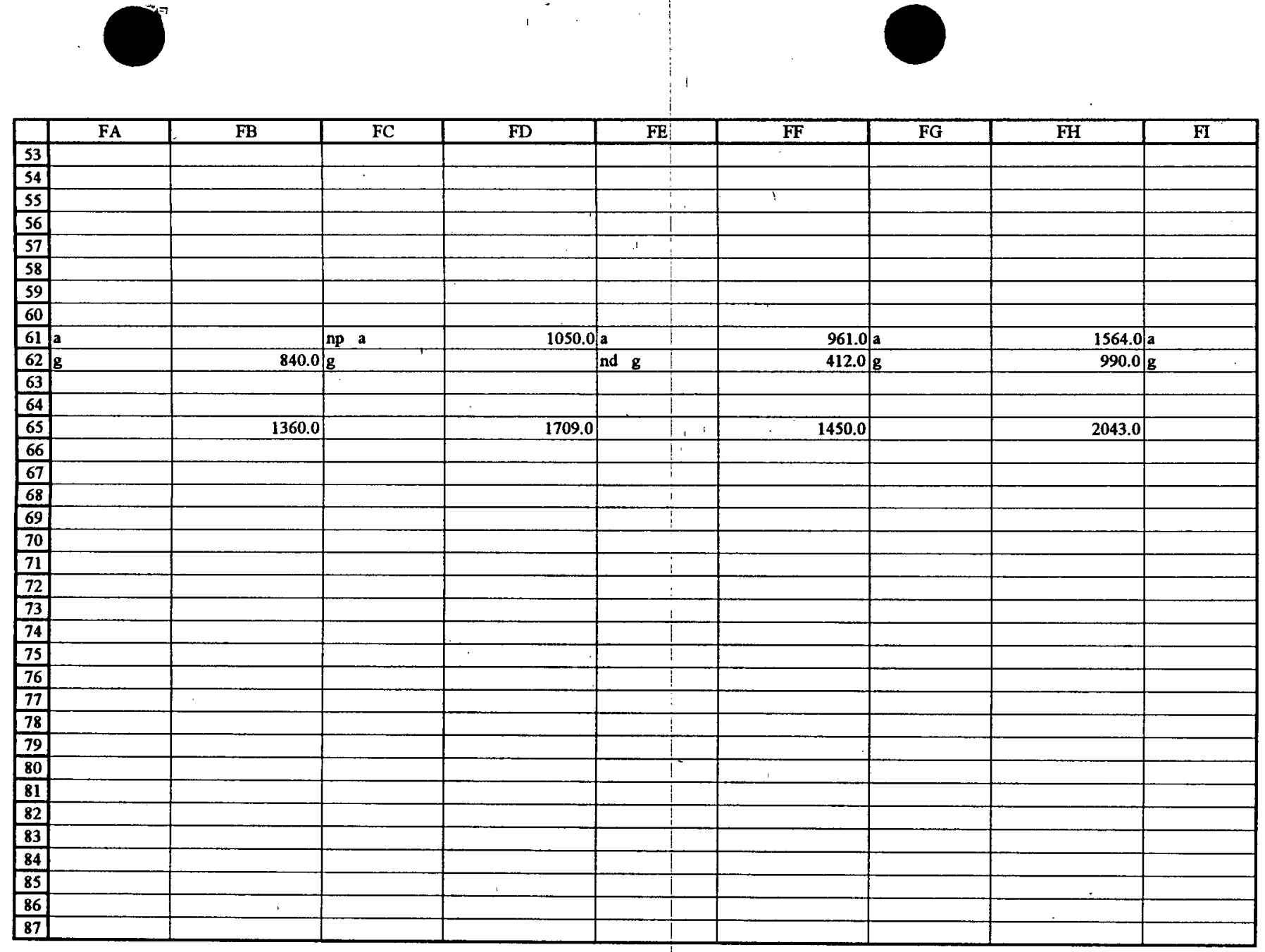


\section{Attachment II}

p. II- 1 of 7

Query Letter and Response from Data Users 
Robert Clayton

07/07/99 12:50 PM

To: $\quad$ Robert Elayer/MM/RWDOE@CRWMS, Jennifer Hinds/YM/RWDOE@CRWMS

cc:

Subject: Tolerance (not social)

Bob and Jennifer,

For some record-keeping we're doing as we qualify the borehole data, I need from you the following information. We have discussed this topic many times in the past, but I need an updated record.

"For your modeling activities, how close do you need the stratigraphic contacts (the input "picks" to the Geologic Framework Model) to be when compared to geophysical logs, core, samples, tests, and/or other downhole data? NOTE: This is a comparison of the picks to other borehole data, not to anything else.

** Please list the GFM strata that are of high importance and those that do not apply to your modeling.

Please be specific to strata and borehole (if necessary) in describing tolerance--for example, Subsurface Design may need closer precision on the lower vitrophyre than on Tptrn, and may not be concerned with boreholes far removed from the repository area.

Also consider this: If your modeling depends only on GFM surfaces (grids) and not on other specific borehole data, the "picks" tolerance probably does not matter in your modeling. I know the UZ model uses some other borehole data as we discussed in relation to UZ-14/UZ-1, but if those data can be placed in your model by strata and not by elevation without adverse effects on your model, tolerance is probably not stringent. That kind of information is central to this data qualification.

Please carefully formulate your reply, as it will influence the format and outcome of our effort to qualify the "picks". For example, if your requirements are plus-or-minus 5 feet and we find a contact outside that range when plotted on the geophysical logs, we will call it non-Q.

Please reply with history by Friday July 9th. Contact me with any questions.

Thanks very much,

$$
\text { -=- Robb }=-
$$

Stratigraphic Contacts Qualification Team Leader

and Geologic Janitor 


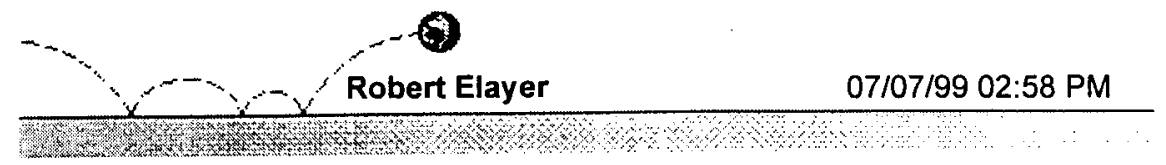

To: Robert Clayton/YM/RWDOE@CRWMS

cc:

Subject: Re: Tolerance (not social) 珷

Gee, I wish I could remember exactly what number I gave during our previous discussions. What I am about to say is based entirely on engineering judgement. I am sure that for your determination of Q-ness based on needs of design and the UZ folks you will need some basis or justification for the numbers. I will try and give this, but it is still all based on engineering judgement.

1. The only boreholes we have a direct interest are the ones within the repository siting area of the central block and those that penetrate through all or part of the repository host horizon.

2. The stratigraphic section we have a direct interest include the units down from the surface to the upper part of the CHn thermal/mechanical unit. Specifically, these include the TCw, PTn, TSw1, TSw2, TSw3, and top of $\mathrm{CHn}$ thermal/mechanical units.

3. Our top contact position tolerance for each lithostratigraphic unit within the above stratigraphic section is listed as follows

TCw

PTn

undiff. units $\quad+-15 \mathrm{ft}$ Importance to shaft and ramp siting and sealing only.

- top

$+-10 \mathrm{ft}$. The nonwelded nature of these units make the location of the top and bottom contacts critical to shaft and ramp design and sealing.

- units within - $\quad+-15 \mathrm{ft}$ These units are variable in their thickness and distribution. They are not individually important to design except collectively as a total PTn unit.

TSw1

- Tptrv1 +-10ft Importance of this unit is that it defines the bottom of the nonwelded PTn units which is critical to shaft and slope design and sealing.

- Tptrn the the is above the repository and is not critical to design except in shaft and slope design.

- Tptrl +-15ft This is above the repository and is not critical to design except in shaft and slope design.

- Tptpul +-15ft This contact between the crystal-rich (above) and crystal-poor (below) is not critical to design.

- top of RHH $\quad+15 \mathrm{ft}$ This gradational contact currently defines the upper limit of the

$\underline{\text { TSw2 }}$ repository siting volume, but this may be eliminated as a constraint in future work.

- Tptpmn +-15ft Gradational contact is not critical to design.

- Tptpll +-15ft Contact is not critical to design.

- Tptpin +-15ft Gradational and often times poorly defined contact is not critical

$\underline{\text { TSw3 }}$ to design.

- Tptpv3 +-5ft This is probably our most critical contact to know because the repository should avoid this vitrophyre unit.

$\underline{\mathrm{CHn}}$

- Tptpv2 +-15ft This defines the top of the nonwelded units in the $\mathrm{CHn}$, but the repository will not penetrate to these depths, so it is not critical to design. 
Hope this is what you needed. I will be around tomorrow (Thursday) then will be out of town for 3-4 weeks (Phase II of our Idaho home). See Bob Saunders if you need to contact me. Cheers!

Robert Clayton

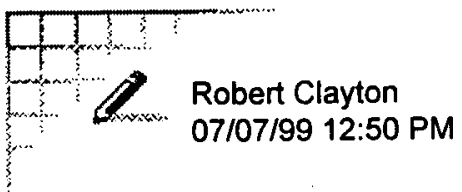

To: $\quad$ Robert Elayer/YM/RWDOE@CRWMS, Jennifer Hinds/YM/RWDOE@CRWMS

cc:

Subject: Tolerance (not social)

Bob and Jennifer,

For some record-keeping we're doing as we qualify the borehole data, I need from you the following information. We have discussed this topic many times in the past, but I need an updated record.

"For your modeling activities, how close do you need the stratigraphic contacts (the input "picks" to the Geologic Framework Model) to be when compared to geophysical logs, core, samples, tests, and/or other downhole data? NOTE: This is a comparison of the picks to other borehole data, not to anything else.

** Please list the GFM strata that are of high importance and those that do not apply to your modeling.

Please be specific to strata and borehole (if necessary) in describing tolerance--for example, Subsurface Design may need closer precision on the lower vitrophyre than on Tptrn, and may not be concerned with boreholes far removed from the repository area.

Also consider this: If your modeling depends only on GFM surfaces (grids) and not on other specific borehole data, the "picks" tolerance probably does not matter in your modeling. I know the UZ model uses some other borehole data as we discussed in relation to UZ-14/UZ-1, but if those data can be placed in your model by strata and not by elevation without adverse effects on your model, tolerance is probably not stringent. That kind of information is central to this data qualification.

Please carefully formulate your reply, as it will influence the format and outcome of our effort to qualify the "picks". For example, if your requirements are plus-or-minus 5 feet and we find a contact outside that range when plotted on the geophysical logs, we will call it non-Q.

Please reply with history by Friday July 9th. Contact me with any questions.

Thanks very much, -=- Robb -=-

Stratigraphic Contacts Qualification Team Leader and Geologic Janitor 
July 7, 1999

Robb,

Here are LBNL's responses to your GFM3.1 - data qualification - tolerance queries. Please give me a call when you've had a chance to look this over.

-Jennifer

*For your modeling activities, how close do you need the stratigraphic contacts (the input "picks" to the Geologic Framework Model) to be when compared to geophysical logs, core, samples, tests, and/or other downhole data? NOTE: This is a comparison of the picks to other borehole data, not to anything else.

The core based contacts are probably the most important from a data perspective.

Please be specific to strata and borehole (if necessary) in describing tolerance.... If your modeling depends only on GFM surfaces (grids) and not on other specific borehole data, the "picks" tolerance probably does not matter in your modeling.... Please carefully formulate your reply, as it will influence the format and outcome of our effort to qualify the "picks". For example, if your requirements are plus-or-minus 5 feet and we find a contact outside that range when plotted on the geophysical logs, we will call it non- $Q$.

Well, I hate to be the bearer of unpleasant news, but we do, in fact, use many of the borehole stratigraphic picks for our work with the UZ model; thus, they will need to be evaluated for qualification. These contact elevations may be very important since they define intervals within which rock properties data (given to us as well ID, elevation or depth, and property value) and perched water data are grouped/assigned.

Bo suggests that a tolerance of plus-or-minus 5 meters (everywhere) is sufficient when comparing the picks to other borehole data, especially for the thicker, repository layers, which are most important in our modeling studies. Does this number sound reasonable to you, or do you feel another value would be more appropriate? Regardless of the tolerance we agree upon, justification of our selection must be documented. This would likely be done with UZ model sensitivity studies, and it is something that we would want to begin fairly soon. Let me know what you think about this. I believe a joint effort is our only way to see this through successfully.

In the meanwhile, we hope to narrow the scope of your efforts a bit with the information provided below. We may be able to reduce this list somewhat (though probably not drastically) by looking through all of the rock property data used as input to the UZ model and their precise elevation/depth, thus omitting layer picks for certain boreholes if no data were collected for that particular layer. Given the short notice and other schedules to work around, we have not yet had the chance to thoroughly go through each borehole data set. If, indeed, this is what you require, we may be able to complete that process next week. 


\section{** Please list the GFM strata that are of high importance and those that do not apply to your modeling.}

The table below lists the GFM3.1 stratigraphic intervals used in the UZ model and the boreholes for which the GFM3.1 file "pix99el.dat" was used in UZ model development. In addition to the file "pix99el.dat," GFM3.1 isochores for each of these units (except for Tpcr and Tpcp) were used in UZ model development, as were the elevation files for the bedrock surface, the upper Tpcp, and upper Tpbt4 contacts.

SD-6, SD-7, SD-9, SD-12, UZ-7a, UZ-14, UZ\#16, and WT\#24 are the most important boreholes because these are where the core saturation data come from. All except SD-6, UZ-7a, and WT\#24 have data from the bedrock surface to the bottom of the borehole. For SD-6, we have data from depths $110 \mathrm{~m}$ to $171 \mathrm{~m}$ and $383 \mathrm{~m}$ to $653 \mathrm{~m}$. For UZ-7a, we have data from depths $39 \mathrm{~m}$ to $235 \mathrm{~m}$. For WT\#24, we have data from depths $514 \mathrm{~m}$ to $770 \mathrm{~m}$. Other boreholes where we have in-situ data are NRG\#4, NRG\#5, NRG-6, NRG-

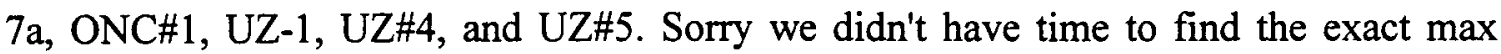
depth for the data from these boreholes, but none of them go beyond the bottom of the Topopah.

\begin{tabular}{|c|l|}
\hline $\begin{array}{c}\text { GFM3.1 } \\
\text { Strata }\end{array}$ & \multicolumn{1}{|c|}{ Boreholes for which "pix99el.dat" was used } \\
\hline Tpcr & SD-6 \\
\hline Tpcp & NRG-7a, SD-7, SD-9, SD-12, UZ\#4, UZ-7a, UZ\#16 \\
\hline TpcLD & unit combined with Tpcp in UZ model; thus, layer pick is not crucial \\
\hline Tpcpv3 & NRG\#5, NRG-6, NRG-7a, SD-6, SD-7, SD-9, SD-12, UZ\#4, UZ-7a, UZ\#16 \\
\hline Tpcpv2 & unit combined with Tpcpv3 in UZ model; thus, layer pick is not crucial \\
\hline Tpcpv1 & NRG\#5, NRG-6, NRG-7a, SD-6, SD-7, SD-9, SD-12, UZ\#4, UZ-7a, UZ\#16 \\
\hline Tpbt4 & NRG\#5, NRG-6, NRG-7a, SD-6, SD-7, SD-9, SD-12, UZ\#4, UZ-7a, UZ\#16 \\
\hline Tpy & NRG\#5, NRG-6, NRG-7a, SD-6, SD-7, SD-9, SD-12, UZ\#4, UZ-7a, UZ\#16 \\
\hline Tpbt3 & NRG\#5, NRG-6, NRG-7a, SD-6, SD-7, SD-9, SD-12, UZ\#4, UZ-7a, UZ\#16 \\
\hline Tpp & NRG\#5, NRG-6, NRG-7a, SD-6, SD-7, SD-9, SD-12, UZ\#4, UZ-7a, UZ\#16 \\
\hline Tpbt2 & NRG\#5, NRG-6, NRG-7a, SD-6, SD-7, SD-9, SD-12, UZ\#4, UZ-7a, UZ\#16 \\
\hline Tptrv3 & Unit is combined with Tpbt2 in UZ model; thus, layer pick is not crucial \\
\hline Tptrv2 & Unit is combined with Tpbt2 in UZ model; thus, layer pick is not crucial \\
\hline Tptrv1 & NRG\#5, NRG-6, NRG-7a, SD-6, SD-7, SD-9, SD-12, UZ\#4, UZ-7a, UZ\#16 \\
\hline Tptrn & NRG\#5, NRG-6, NRG-7a, SD-6, SD-7, SD-9, SD-12, UZ\#4, UZ-7a, UZ\#16 \\
\hline Tptr1 & NRG\#5, NRG-6, NRG-7a, SD-6(?), SD-7, SD-9, SD-12, UZ-7a, UZ\#16 \\
\hline Tptf & Unit is combined with Tptrl in UZ model; thus, layer pick is not crucial \\
\hline Tptpul & Unit is combined with Tptrl in UZ model; thus, layer pick is not crucial \\
\hline Tptpmn & NRG\#5, NRG-6, NRG-7a, SD-6(?), SD-7, SD-9, SD-12, UZ-7a, UZ\#16 \\
\hline TptpII & NRG\#5, NRG-6, NRG-7a, SD-6, SD-7, SD-9, SD-12, UZ-7a, UZ\#16 \\
\hline Tptpln & NRG\#5, NRG-7a, SD-6, SD-7, SD-9, SD-12, UZ\#16 \\
\hline Tptpv3 & NRG-7a, SD-6, SD-7, SD-9, SD-12, UZ\#16, WT\#24 \\
\hline Tptpv2 & NRG-7a, SD-6, SD-7, SD-9, SD-12, UZ-14, UZ\#16, WT\#24 \\
\hline Tptpv1 & NRG-7a, SD-6, SD-7, SD-9, SD-12, UZ-14, UZ\#16, WT\#24 \\
\hline Tpbt1 & Unit is combined with Tptpv1 in UZ model; thus, layer pick is not crucial \\
\hline Tac & NRG-7a, SD-6, SD-7, SD-9, SD-12, UZ-14, UZ\#16, WT\#24, G-2 \\
\hline Tacbt & SD-6, SD-7, SD-9, SD-12, UZ-14, UZ\#16 \\
\hline Prowuv & SD-6, SD-7, SD-9, SD-12, UZ-14, UZ\#16 \\
\hline Prowuc & SD-6, SD-7, SD-9, SD-12, UZ-14, UZ\#16 \\
\hline
\end{tabular}




\begin{tabular}{|c|l|}
\hline $\begin{array}{c}\text { GFM3.1 } \\
\text { Strata }\end{array}$ & \multicolumn{1}{|c|}{ Boreholes for which "pix99el.dat" was used } \\
\hline Prowmd & SD-6, SD-7, SD-12, UZ-14, UZ\#16 \\
\hline Prowlc & unit is combined with Prowmd in UZ model; thus, layer pick is not crucial \\
\hline Prowlv & SD-6, SD-7, SD-12, UZ-14 \\
\hline Prowbt & unit is combined with Prowlv in UZ model; thus, layer pick is not crucial \\
\hline Bulluv & unit is combined with Prowlv in UZ model; thus, layer pick is not crucial \\
\hline Bulluc & SD-6 \\
\hline Bullmd & unit is combined with Bulluc in UZ model; thus, layer pick is not crucial \\
\hline Bulllc & unit is combined with Bulluc in UZ model; thus, layer pick is not crucial \\
\hline Bulllv & N/A, no rock property data \\
\hline Bullbt & unit is combined with Bulllv in UZ model; thus, layer pick is not crucial \\
\hline Tramuv & unit is combined with Bulllv in UZ model; thus, layer pick is not crucial \\
\hline Tramuc & N/A, no rock property data \\
\hline Trammd & unit is combined with Tramuc in UZ model; thus, layer pick is not crucial \\
\hline Tramlc & unit is combined with Tramuc in UZ model; thus, layer pick is not crucial \\
\hline
\end{tabular}

Layers Bullbt through Tramlc are included in the UZ model where they lie above the water table, but their exact contact elevations are not essential since little or no data exist to characterize them hydrogeologically.

GFM3.1 strata that DO NOT apply to UZ modeling:

$\mathrm{RHH}$

Tund (older Tertiary)

Paleozoic 
TDR-NBS-GS-000007

Attachment III:

p. III- 1 of 2

Cross Section from GFM3.1 Showing Logs 


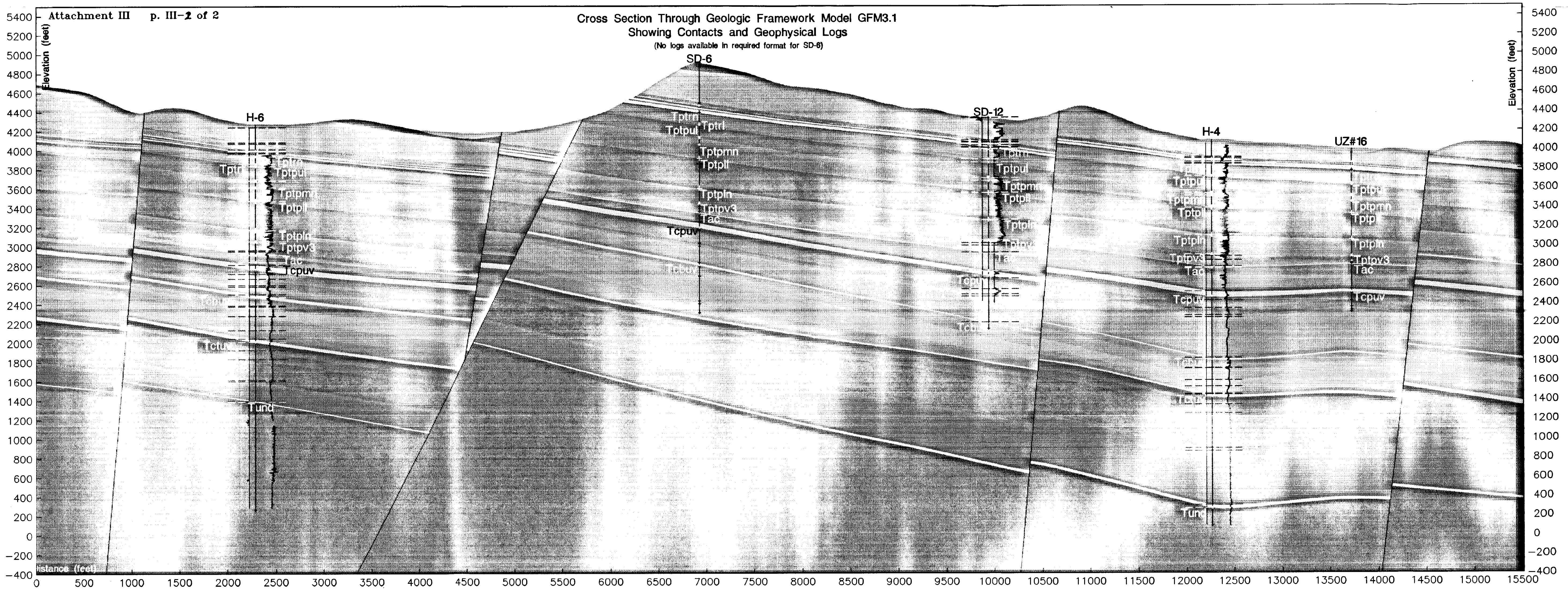


Attachment IV:

p. IV-1 of 5

Thickness Maps 


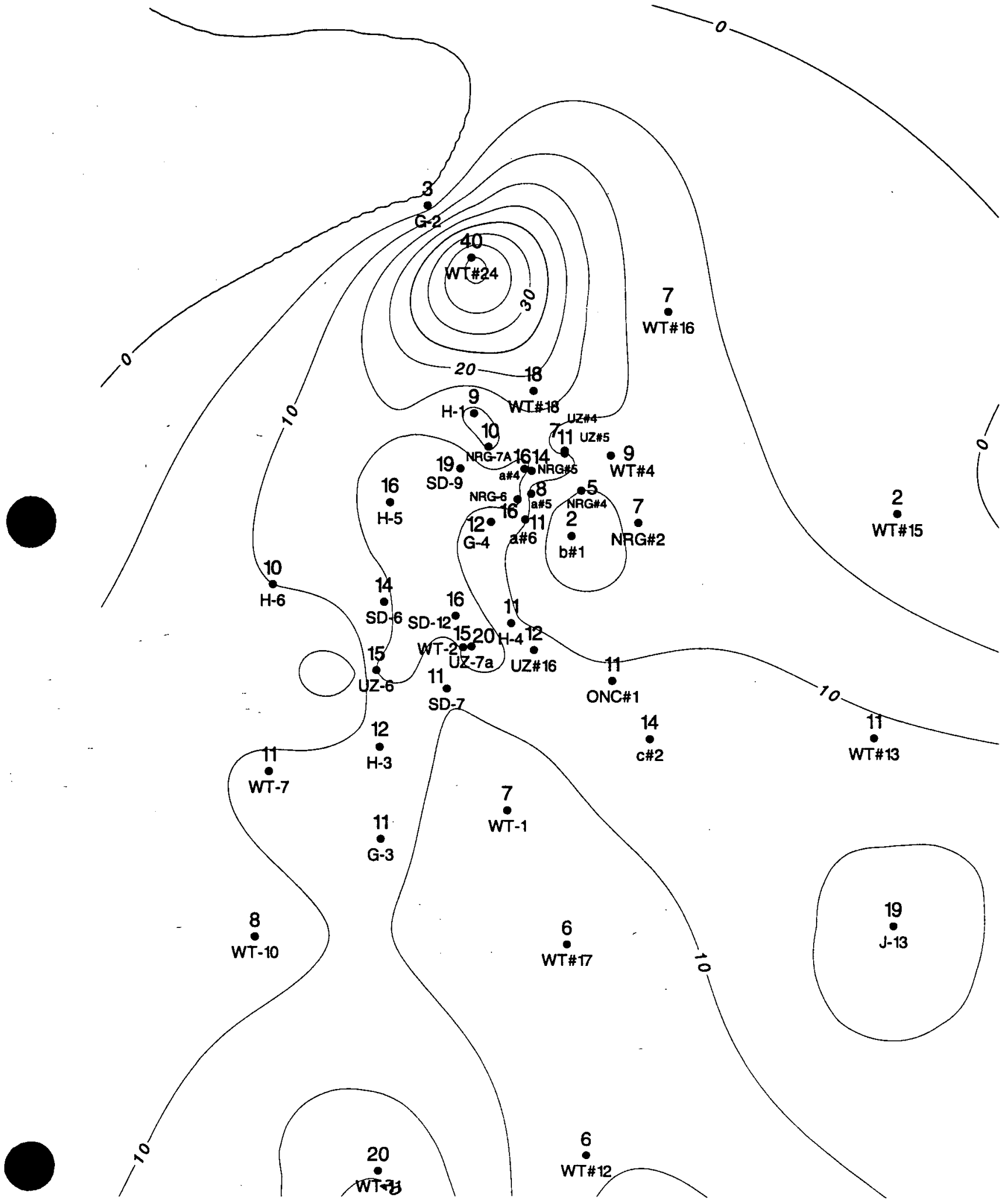


contour interval 5 feet
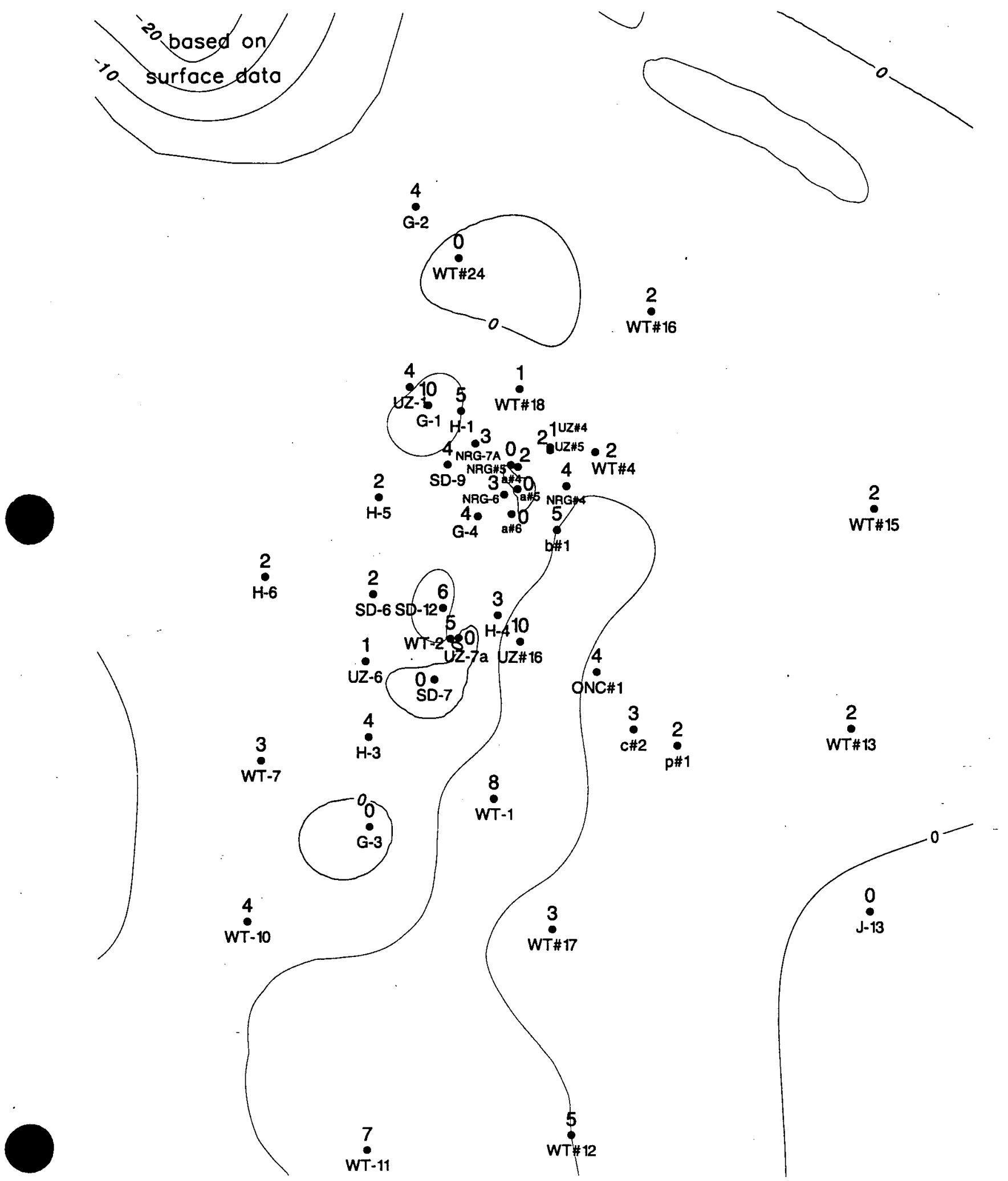
Attachment IV: Thickness maps Thickness of Tptpv3 contour interval 10 feet

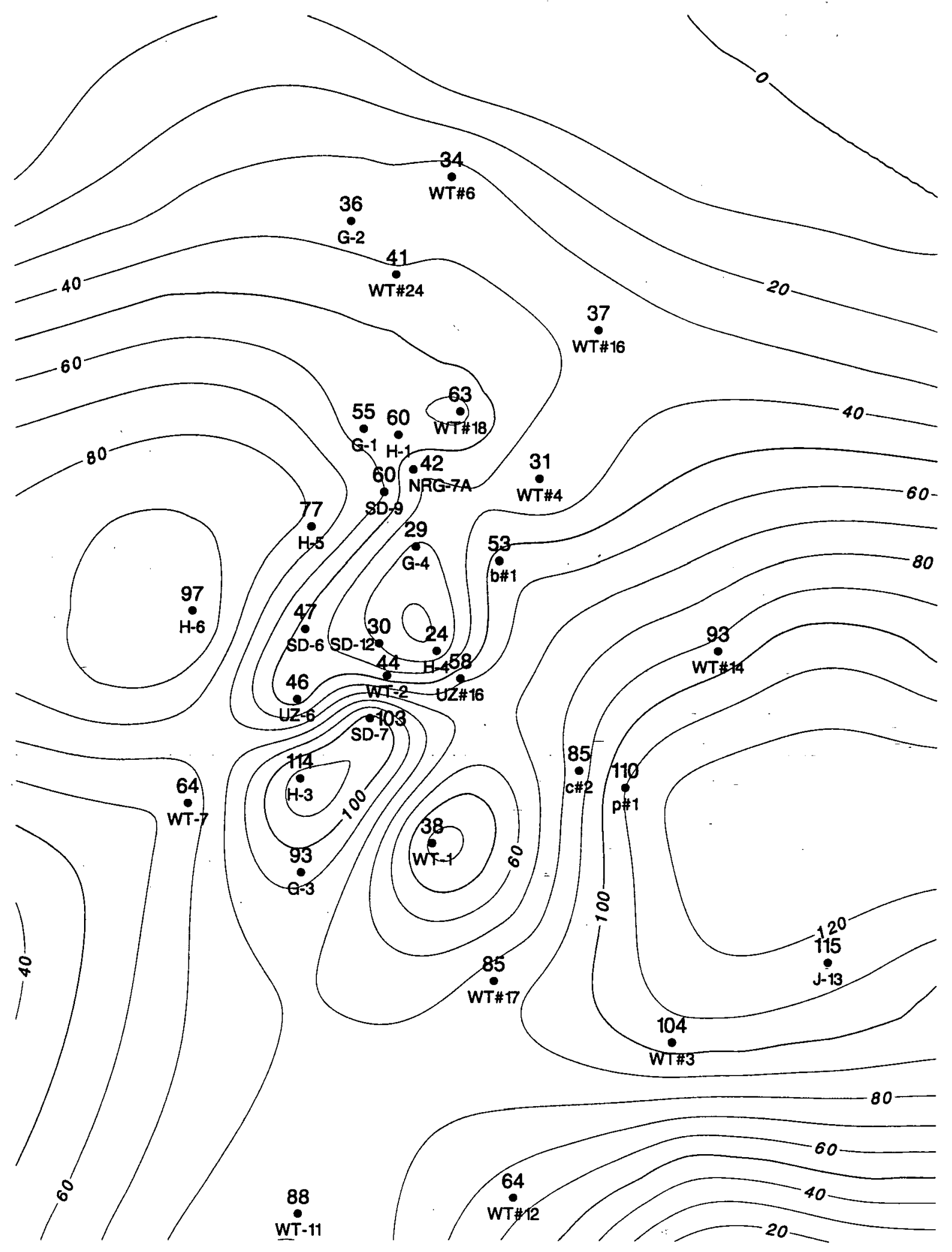


Thickness of Tptpv2

contour interval 10 feet

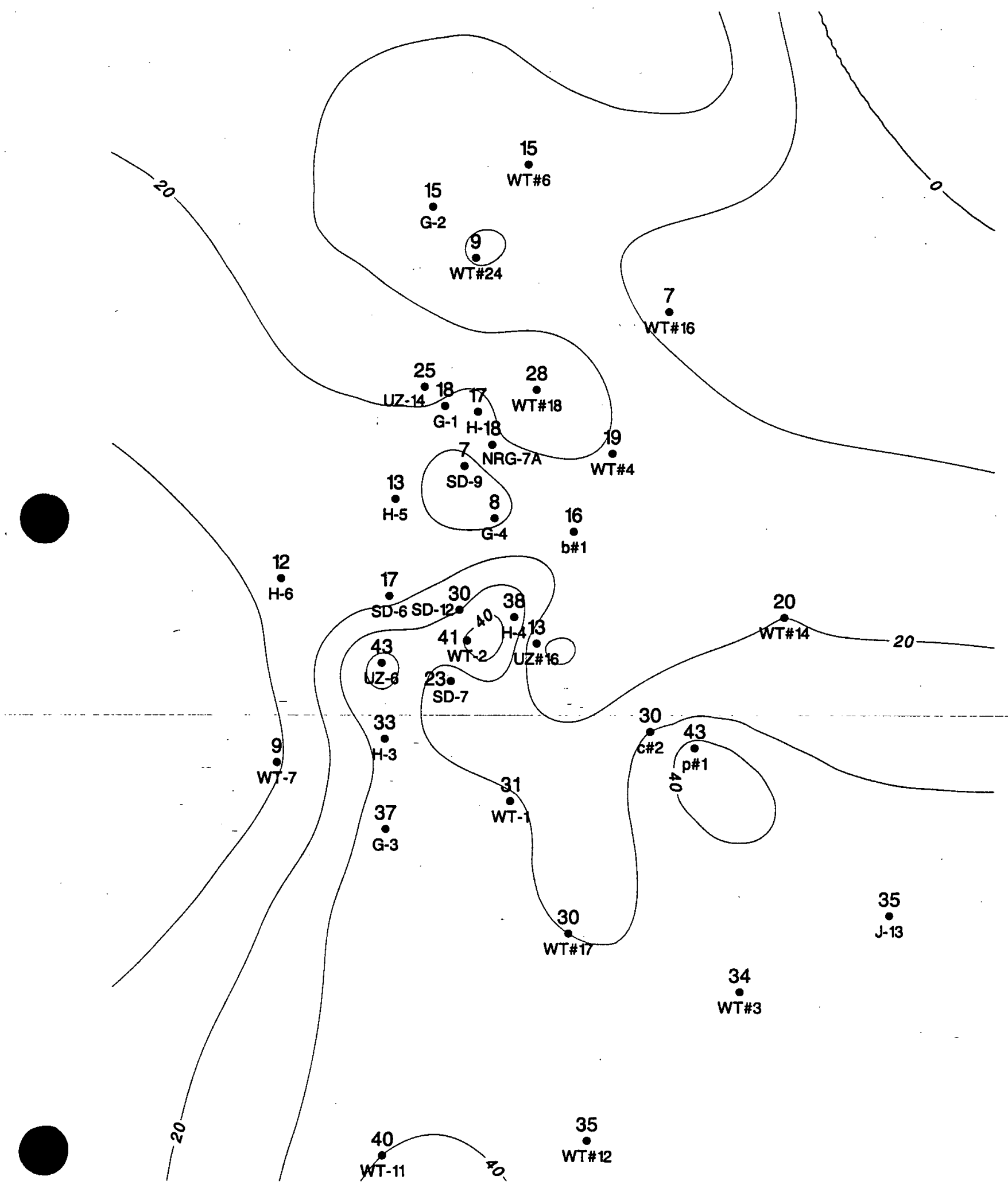


Attachment V:

p. $\mathrm{V}-1$ of 2

Typical Geophysical Log Signatures for the Sample Contacts 
TDR-NBS-GS-000007

p. VI-1 of 8

\section{Attachment VI: \\ Contacts Examination Checklist}




\begin{tabular}{|c|c|c|c|c|}
\hline & $\bar{A}$ & B & C & $\mathrm{D}$ \\
\hline 1 & \multicolumn{4}{|c|}{ Appendix VI: Contacts Examination Checklist } \\
\hline 2 & \multicolumn{4}{|c|}{ Data Qualification Report: Borehole Stratigraphic Contacts Data } \\
\hline 3 & Borehole & Contact & Status & Justification \\
\hline 4 & a\#1 & Tpcpv2 & NL & No donsity logi \\
\hline 5 & a\#1 & Tptrv1 & NL & J fould tie made based on Gimma toy lug; acoptable contact \\
\hline 6 & a\#1 & Tptpv3 & $\checkmark$ & $20, \ldots 1,1,1,16$ \\
\hline 7 & a\#1 & Tptpv2 & 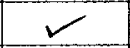 & 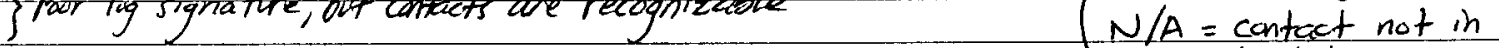 \\
\hline 8 & a\#4 & Tpcpv2 & $\checkmark$ & borehole \\
\hline 9 & a\#4 & Tptrv1 & $\checkmark$ & 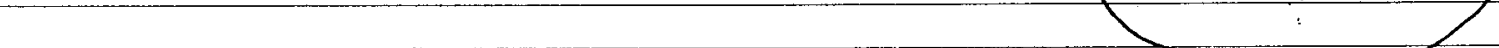 \\
\hline 10 & a\#4 & Tptpv3 & $N / A$ & \\
\hline 11 & a\#4 & Tptpv2 & $N / A$ & $\therefore$ \\
\hline 12 & a\#5 & Tpcpv2 & $\checkmark$ & \\
\hline 13 & a\#5 & Tptrv1 & 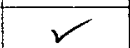 & \\
\hline 14 & a\#5 & Tptpv3 & $N / A$ & 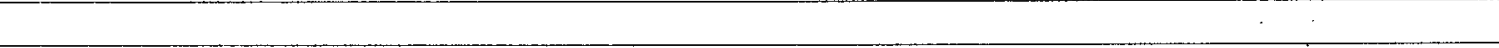 \\
\hline 15 & a\#5 & Tptpv2 & $N / A$ & \\
\hline 16 & a\#6 & Tpcpv2 & $\checkmark$ & \\
\hline 17 & a\#6 & Tptrv1 & $\checkmark$ & . \\
\hline 18 & a\#6 & Tptpv3 & $N / A$ & \\
\hline 19 & a\#6 & Tptpv2 & $N / A$ & \\
\hline 20 & a\#7 & Tpcpv2 & $\checkmark$ & Noisy log, but passable \\
\hline 21 & a\#7 & Tṕtrv1 & $\checkmark$ & \\
\hline 22 & a\#7 & Tptpv3 & $N / A$ & \\
\hline 23 & a\#7 & Tptpv2 & $N / A$ & \\
\hline 24 & $b \# 1$ & Tpcpv2 & NL & No logs; existent logs are very poor; other ckata may be available \\
\hline 25 & $\mathrm{b \# 1}$ & Tptrv1 & NL & No logs; existent logs are very poor; other dita mm be avaibable. \\
\hline 26 & b\#1 & Tptpv3 & ? okay & 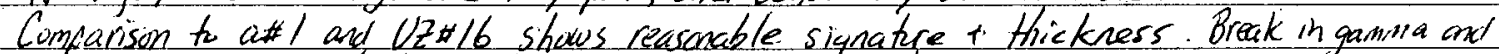 \\
\hline 27 & b\#1 & Tptpv2 & $\checkmark$ & 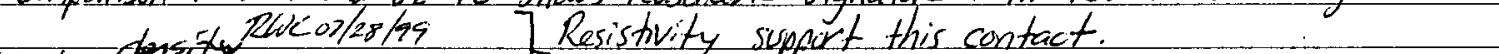 \\
\hline 28 & c\#1 & Tpcpv2 & NLV & Wo indelug ime \\
\hline 29 & C\#1 & Tptrv1 & $\checkmark$ & Consistent with GimmsaPay \\
\hline 30 & c\#1 & Tptpv3 & $\checkmark$ & Plakcon/28/95 \\
\hline 31 & $c \# 1$ & Tptpv2 & 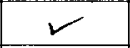 & 2 \\
\hline 32 & c\#2 & Tpcpv2 & NLV & No dionatiogs? \\
\hline 33 & C\#2 & Tptrv1 & $N L V$ & Nodompostic logs Jontacts a \\
\hline 34 & $c \# 2$ & Tptpv3 & $\checkmark$ & \\
\hline 35 & $\mathrm{c} \# 2$ & Tptpv2 & 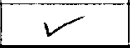 & $\because$ \\
\hline
\end{tabular}




\begin{tabular}{|c|c|c|c|c|}
\hline & A & B & C & D \\
\hline 36 & c\#3 & Tpcpv2 & NLV & In density bus-made based on resistrith breaks \\
\hline 37 & $c \# 3$ & Tptrv1 & NLV & 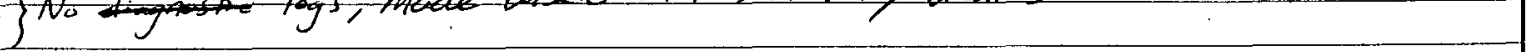 \\
\hline 38 & $c \# 3$ & Tptpv3 & $\checkmark$ & \\
\hline 39 & $c \# 3$ & Tptpv2 & $\checkmark$ & \\
\hline 40 & G-1 & Tpcpv2 & $N / A$ & \\
\hline 41 & G-1 & Tptrv1 & NL & \\
\hline 42 & G-1 & Tptpv3 & $\checkmark$ & subtly expressed. Density is same as vionlithophysal intervals \\
\hline 43 & G-1 & Tptpv2 & $\checkmark$ & \\
\hline 44 & G-2 & Tpcpv2 & $N L$ & \\
\hline 45 & G-2 & Tptrv1 & $\checkmark$ & \\
\hline 46 & G-2 & Tptpv3 & $\checkmark$ & Low clansity /Wushent is within 5 ft of contact \\
\hline 47 & G-2 & Tptpv2 & $\checkmark$ & \\
\hline 48 & G-3 & Tpсpv2 & $\checkmark$ & \\
\hline 49 & G-3 & Tptrv1 & $\checkmark$ & \\
\hline 50 & G-3 & Tptpv3 & $\checkmark$ & \\
\hline 51 & G-3 & Tptpv2 & $\checkmark$ & \\
\hline 52 & G-4 & Tpcpv2 & $\checkmark$ & Hole washed out, but contact is at slope change \\
\hline 53 & G-4 & Tptrv1 & $\checkmark$ & This antact pushes the $\pm 10 \mathrm{ft}$ window, but is within it \\
\hline 54 & G-4 & Tptpv3 & $\checkmark$ & Confined by break in Gaimma Ray loy \\
\hline 55 & G-4 & Tptpv2 & $\checkmark$ & \\
\hline 56 & $\mathrm{H}-1$ & Tpcpv2 & NL & No $\log r$ \\
\hline 57 & $\mathrm{H}-1$ & Tptrv1 & NL & No logs \\
\hline 58 & $\mathrm{H}-1$ & Tptpv3 & $\checkmark$ & in tox of vitophyre \\
\hline 59 & $\mathrm{H}-1$ & Tptpv2 & 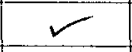 & \\
\hline 60 & $\mathrm{H}-3$ & Tpcpv2 & $\checkmark$ & \\
\hline 61 & $\mathrm{H}-3$ & Tptrv1 & $\simeq$ & \\
\hline 62 & $\mathrm{H}-3$ & Tptpv3 & ?okay & Por log + whshut. Low resistrvity is dragnestic of this init's tep. Vitrophype \\
\hline 63 & $\mathrm{H}-3$ & Tptpv2 & $r$ & Ito have thick and thin peots - this cuntact is reasonable? Depth besed an \\
\hline 64 & $\mathrm{H}-4$ & Tpcpv2 & $\checkmark$ & resistivity. \\
\hline 65 & $\mathrm{H}-4$ & Tptrv1 & $\checkmark$ & \\
\hline 66 & $\mathrm{H}-4$ & Tptpv3 & ?okay & Pow loust +ukiskurt. Thickness consistent with G-4 and SO-12. Consistent with Resistivity \\
\hline 67 & $\mathrm{H}-4$ & Tptpv2 & ? okay & Ror kuss + lkashurt. Thickness consistent with WT-2 and SD-12. Consistent with Resistrirk \\
\hline 68 & $\mathrm{H}-5$ & Tpcpv2 & $\checkmark$ & \\
\hline 69 & $\mathrm{H}-5$ & Tptrv1 & $\checkmark$ & \\
\hline 70 & $\mathrm{H}-5$ & Tptpv3 & $\checkmark$ & \\
\hline 71 & $\mathrm{H}-5$ & Tptpv2 & $\checkmark$ & \\
\hline
\end{tabular}




\begin{tabular}{|c|c|c|c|c|}
\hline & $\bar{A}$ & $\mathrm{~B}$ & $\mathrm{C}$ & $\mathbf{D}$ \\
\hline 72 & $\mathrm{H}-6$ & Tpcpv2 & NL & Nu diagnostic logs \\
\hline 73 & H-6 & Tptrv1 & $\checkmark$ & pule $\rightarrow 27 / 279$ \\
\hline 74 & $\mathrm{H}-6$ & Tptpv3 & $\checkmark$ & Very thick but consistent with Rou + Resistwiz break \\
\hline 75 & $\mathrm{H}-6$ & Tptpv2 & $\checkmark$ & \\
\hline 76 & $\mathrm{~J}-13$ & Tpcpv2 & $\checkmark$ & Gamma a Desistivity break \\
\hline 77 & $\mathrm{~J}-13$ & Tptrv1 & $\checkmark$ & Cramma + Resistrity break \\
\hline 78 & $\mathrm{~J}-13$ & Tptpv3 & 2 & Gainna + Resisturity i smooth cunve is diagnostic \\
\hline 79 & $\mathrm{~J}-13$ & Tptpv2 & $\checkmark$ & Gamma + Resistivith break \\
\hline 80 & NRG\#1 & Tpcpv2 & $N / A$ & \\
\hline 81 & NRG\#1 & Tptrv1 & $N / A$ & No loas available \\
\hline 82 & NRG\#1 & Tptpv3 & $N / A$ & 7 \\
\hline 83 & NRG\#1 & Tptpv2 & $N / A$ & \\
\hline 84 & NRG\#2 & Tpcpv2 & $N L$ & \\
\hline 85 & NRG\#2 & Tptrv1 & $N / A$ & \\
\hline 86 & NRG\#2 & Tptpv3 & $N / A$ & \\
\hline 87 & NRG\#2 & Tptpv2 & $N / A$ & \\
\hline 88 & NRG\#2b & Tpсpv2 & $N / A$ & $i$ \\
\hline 89 & NRG\#2b & Tptrv1 & $N / A$ & 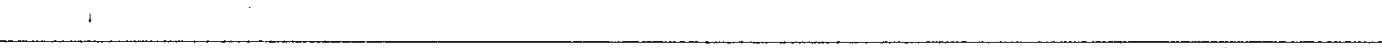 \\
\hline 90 & NRG\#2b & Tptpv3 & $N / A$ & \\
\hline 91 & NRG\#2b & Tptpv2 & $N / A$ & 1 \\
\hline 92 & NRG\#4 & Tpcpv2 & $\checkmark$ & 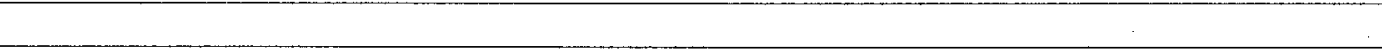 \\
\hline 93 & NRG\#4 & Tptrv1 & $\checkmark$ & \\
\hline 94 & NRG\#4 & Tptpv3 & $N / A$ & \\
\hline 95 & NRG\#4 & Tptpv2 & $N / A$ & \\
\hline 96 & NRG\#5 & Tpcpv2 & $\checkmark$ & Log through casing but pattem appers to be correct \\
\hline 97 & NRG\#5 & Tptrv1 & $\checkmark$ & \\
\hline 98 & NRG\#5 & Tptpv3 & $N / A$ & \\
\hline 99 & NRG\#5 & Tptpv2 & $N / A$ & \\
\hline 100 & NRG\#6 & Tpcpv2 & $\checkmark$ & \\
\hline 101 & NRG\#6 & Tptrv1 & $\checkmark$ & . \\
\hline 102 & NRG\#6 & Tptpv3 & $N / A$ & \\
\hline 103 & NRG\#6 & Tptpv2 & $N / A$ & \\
\hline
\end{tabular}




\begin{tabular}{|c|c|c|c|c|}
\hline & $\bar{A}$ & $\mathrm{~B}$ & $\mathrm{C}$ & $\mathbf{D}$ \\
\hline 104 & NRG\#7 & Tpcpv2 & $\checkmark$ & \\
\hline \begin{tabular}{|l|}
105 \\
\end{tabular} & NRG\#7 & Tptrv1 & $\checkmark$ & $:$ \\
\hline 106 & NRG\#7 & Tptpv3 & NL & No $\log 5$ \\
\hline 107 & NRG\#7 & Tptpv2 & NL & No logs \\
\hline 108 & ONC\#1 & Tpcpv2 & $\checkmark$ & \\
\hline 109 & ONC\#1 & Tptrv1 & $\checkmark$ & \\
\hline 1110 & ONC\#1 & Tptpv3 & $N / A$ & 37 \\
\hline 111 & ONC\#1 & Tptpv2 & $N / A$ & $3^{\text {the }}$ \\
\hline 112 & $\mathrm{p} \# 1$ & Tpcpv2 & $N / A$ & \\
\hline 113 & p\#1 & Tptrv1 & $\checkmark$ & \\
\hline 114 & $p \# 1$ & Tptpv3 & $\checkmark$ & \\
\hline 115 & $p \# 1$ & Tptpv2 & $\checkmark$ & $3^{170}$ \\
\hline 116 & SD-7 & Tpcpv2 & $\checkmark$ & \\
\hline 1117 & SD-7 & Tptrv1 & $\checkmark$ & \\
\hline 118 & $\frac{S D-7}{C D}$ & Tptpv3 & $\checkmark$ & As in many holes, cov? here has a distinct low-density layer in its top \\
\hline 119 & SD-7 & Tptpv2 & $\checkmark$ & \\
\hline 120 & SD-9 & Tpcpv2 & $\checkmark$ & Log signature not good qualify, but is acceptable \\
\hline \begin{tabular}{|l|}
121 \\
\end{tabular} & SD-9 & Tptrv1 & $r$ & \\
\hline 122 & SD-9 & Tptpv3 & $\checkmark$ & Low density in top of vitrophyre \\
\hline 123 & SD-9 & Tptpv2 & $\checkmark$ & \\
\hline \begin{tabular}{|l|}
124 \\
\end{tabular} & SD-12 & Tpcpv2 & $\checkmark$ & 7 \\
\hline 125 & SD-12 & Tptrv1 & $\checkmark$ & These are the type \\
\hline 126 & SD-12 & Tptpv3 & $\checkmark$ & \\
\hline 127 & SD-12 & Tptpv2 & $\checkmark$ & T \\
\hline 128 & $\mathrm{UZ}-1$ & Tpcpv2 & $N / A$ & \\
\hline 129 & UZ-1 & Tptrv1 & $\checkmark$ & \\
\hline 130 & UZ-1 & Tptpv3 & $N L$ & Not logged \\
\hline 131 & $\mathrm{UZ}-1$ & Tptpv2 & $N L$ & Not logged \\
\hline 132 & UZ\#4 & Tpcpv2 & $\checkmark$ & \\
\hline 133 & UZ\#4 & Tptrv1 & $r$ & \\
\hline 134 & UZ\#4 & Tptpv3 & $N / A$ & \\
\hline i 135 & UZ\#4 & Tptpv2 & $N / A$ & \\
\hline
\end{tabular}




\begin{tabular}{|c|c|c|c|c|}
\hline & $A$ & B & $\mathrm{C}$ & D \\
\hline 136 & UZ\#5 & Tpcpv2 & $\checkmark$ & \\
\hline 137 & UZ\#5 & Tptrv1 & $\checkmark$ & , \\
\hline 138 & UZ\#5 & Tptpv3 & $N / A$ & 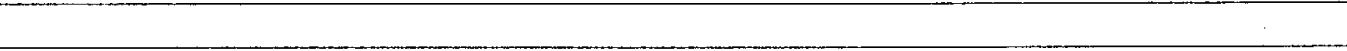 \\
\hline 139 & UZ\#5 & Tptpv2 & $N / A$ & \\
\hline 140 & UZ-6 & Tpcpv2 & $\checkmark$ & \\
\hline 141 & UZ-6 & Tptrv1 & $\checkmark$ & \\
\hline 142 & UZ-6 & Tptpv3 & $\checkmark$ & Top cansistent with GammadResistovity breaks \\
\hline 143 & UZ-6 & Tptpv2 & $\checkmark$ & \\
\hline 144 & UZ-7a & Tpcpv2 & $\checkmark$ & \\
\hline 145 & UZ-7a & Tptrv1 & $\checkmark$ & Density log is choppy but contact is consistent with Gauma + Resistwith breaks. \\
\hline 146 & UZ-7a & Tptpv3 & $N / A$ & \\
\hline 147 & UZ-7a & Tptpv2 & $N / A$ & . \\
\hline 148 & UZ-14 & Tpcpv2 & $N / A$ & \\
\hline 149 & UZ-14 & Tptrv1 & NL & No logs \\
\hline 150 & UZ-14 & Tptpv3 & NL & Nologs \\
\hline 151 & UZ-14 & Tptpv2 & $\checkmark$ & \\
\hline 152 & UZ\#16 & Tpcpv2 & $\checkmark$ & \\
\hline 153 & UZ\#16 & Tptrv1 & $\checkmark$ & \\
\hline 154 & UZ\#16 & Tptpv3 & $\checkmark$ & vitrophyre is broken up, but recognizalite \\
\hline 155 & UZ\#16 & Tptpv2 & 2 & \\
\hline 156 & $\mathrm{WT}-1$ & Tpcpv2 & $\checkmark$ & \\
\hline 157 & WT-1 & Tptrv1 & $\checkmark$ & \\
\hline 158 & WT-1 & Tptpv3 & $\checkmark$ & Low density zene is well doveloped. \\
\hline 159 & WT-1 & Tptpv2 & $\checkmark$ & Low slope gradient is consistent with (but subdued from) other holes \\
\hline 160 & WT-2 & Tpcpv2 & $\checkmark$ & \\
\hline 161 & WT-2 & Tptrv1 & $\checkmark$ & \\
\hline 162 & WT-2 & Tptpv3 & $\checkmark$ & \\
\hline 163 & WT-2 & Tptpv2 & $\checkmark$ & \\
\hline 164 & WT\#3 & Tpсpv2 & $N / A$ & \\
\hline 165 & WT\#3 & Tptrv1 & $N / A$ & \\
\hline 166 & WT\#3 & Tptpv3 & $r$ & Lack of deep clansity lows distinquishes fiam normal welded Tet \\
\hline 167 & WT\#3 & Tptpv2 & $\checkmark$ & \\
\hline
\end{tabular}




\begin{tabular}{|c|c|c|c|c|}
\hline & A & $\mathrm{B}$ & $\mathrm{C}$ & D \\
\hline 168 & WT\#4 & Tpcpv2 & $\checkmark$ & Big cavity just above masks the contact, but it's consis tent with the reference (typepection. \\
\hline 169 & WT\#4 & Tptrv1 & $\checkmark$ & 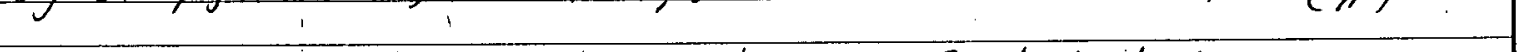 \\
\hline 170 & WT\#4 & Tptpv3 & $\checkmark$ & Unusuil density signature; consistent with Gamma + Resistivity breaks \\
\hline 171 & WT\#4 & Tptpv2 & 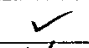 & \\
\hline 172 & WT\#6 & Tpcpv2 & $N / A$ & \\
\hline 173 & WT\#6 & Tptrv1 & $N / A$ & \\
\hline 174 & WT\#6 & Tptpv3 & $\checkmark$ & Well-developed low-density one \\
\hline 175 & WT\#6 & Tptpv2 & $\sqrt{2}$ & : \\
\hline 176 & WT-7 & Tpcpv2 & $\checkmark$ & 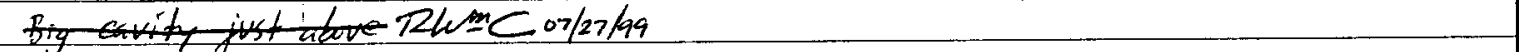 \\
\hline 177 & WT-7 & Tptrv1 & $\checkmark$ & $2-1$ \\
\hline 178 & WT-7 & Tptpv3 & $\checkmark$ & Unusual density log, but consistent with \\
\hline 179 & WT-7 & Tptpv2 & $\checkmark$ & Consistent with Gamma Roy break. \\
\hline 180 & WT-10 & Tpcpv2 & $\checkmark$ & rente \\
\hline 181 & WT-10 & Tptrv1 & $\checkmark$ & \\
\hline 182 & WT-10 & Tptpv3 & $N / A$ & \\
\hline 183 & WT-10 & Tptpv2 & $N / A$ & $:$ \\
\hline 184 & WT-11 & Tpcpv2 & $\checkmark$ & Consistent with Gamma + Tesictrify breakr \\
\hline 185 & WT-11 & Tptrv1 & $\checkmark$ & ( \\
\hline 186 & WT-11 & Tptpv3 & $\checkmark$ & \\
\hline 187 & WT-11 & Tptpv2 & $\checkmark$ & \\
\hline 188 & WT\#12 & Tpcpv2 & $\checkmark$ & $i$ \\
\hline 189 & WT\#12 & Tptrv1 & $\sim$ & \\
\hline 190 & WT\#12 & Tptpv3 & $\longleftarrow$ & Low density znes are within tpr3-lowest high derrity park \\
\hline 191 & WT\#12 & Tptpv2 & $\checkmark$ & \\
\hline 192 & WT\#13 & Tpcpv2 & $\checkmark$ & \\
\hline 193 & WT\#13 & Tptrv1 & $r$ & \\
\hline 194 & WT\#13 & Tptpv3 & $N / A$ & \\
\hline 195 & WT\#13 & Tptpv2 & $N / A$ & \\
\hline 196 & WT\#14 & Tpcpv2 & $N / A$ & \\
\hline 197 & WT\#14 & Tptrv1 & NL & \\
\hline 198 & WT\#14 & Tptpv3 & $\checkmark$ & " \\
\hline 199 & WT\#14 & Tptpv2 & $\checkmark$ & \\
\hline
\end{tabular}




\begin{tabular}{|c|c|c|c|c|}
\hline & A & B & $\mathrm{C}$ & 1 \\
\hline 200 & WT\#15 & Tpcpv2 & $\checkmark$ & 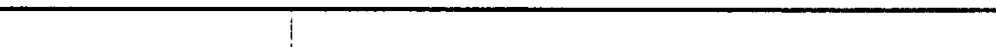 \\
\hline 201 & WT\#15 & Tptrv1 & $\checkmark$ & Barehy within the $10 \mathrm{ft}$ 'window: all loos are add here. \\
\hline 202 & WT\#15 & Tptpv3 & $N / A$ & \\
\hline 203 & WT\#15 & Tptpv2 & $N / A$ & \\
\hline 204 & WT\#16 & Tpcpv2 & 2 & Withn the $10 \mathrm{ft}$ window. \\
\hline 205 & WT\#16 & Tptrv1 & 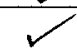 & \\
\hline 206 & WT\#16 & Tptpv3 & $\checkmark$ & \\
\hline 207 & WT\#16 & Tptpv2 & $\checkmark$ & 1 \\
\hline 208 & WT\#17 & Tpcpv2 & $\checkmark$ & Large low-dessity (corroded) Zne above cantact. \\
\hline 209 & WT\#17 & Tptrv1 & 2 & \\
\hline 210 & WT\#17 & Tptpv3 & $\checkmark$ & \\
\hline 211 & WT\#17 & Tptpv2 & $\checkmark$ & $i$ \\
\hline 212 & WT\#18 & Tpcpv2 & $\checkmark$ & . \\
\hline 213 & WT\#18 & Tptrv1 & $\checkmark$ & $i$ \\
\hline 214 & WT\#18 & Tptpv3 & $\checkmark$ & low-density zone in top of vitrophype \\
\hline 215 & WT\#18 & Tptpv2 & 2 & 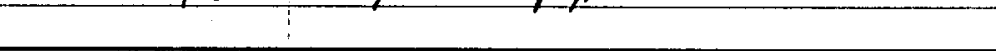 \\
\hline
\end{tabular}


TDR-NBS-GS-000007

\section{Attachment VII:}

Data Recommended to be Qualified

22-page spreadsheet numbered separately 


\begin{tabular}{|c|c|c|c|c|c|}
\hline & $\bar{A}$ & $\mathbf{B}$ & $\mathrm{C}$ & $\mathrm{D}$ & $\mathbf{E}$ \\
\hline 1 & \multicolumn{5}{|c|}{ Re-evaluation of key subsurface lithostratigraphic contacts: FY97 milestone SPG39IM4. Typos and omissions corrected for input to GFM3.0 by R. } \\
\hline 2 & In addition to the lithostratigraphic contacts, the table contains values for the: & & & & \\
\hline 3 & 1. vitric-zeolitic boundary (V-Z) & & Green cells & \multicolumn{2}{|c|}{ Resolution of contacts initially ch } \\
\hline 4 & & & Blue cells & \multicolumn{2}{|c|}{ Modified contacts based on sugg } \\
\hline 5 & 2. type of contact at the top of each unit & & & & \\
\hline 6 & \multicolumn{3}{|c|}{ Repository Host Horizon (RHH) identified by R. Elayer (MK M\&O) based on character of geophysical logs } & & \\
\hline 7 & \multicolumn{2}{|l|}{ Colors of headers indicate: Black - Q-status geophysical logs (mostly) and core or cuttings } & & & \\
\hline 8 & Blue - Q-status core or cuttings, no geophysical lo & & & & \\
\hline 9 & & & & Contact & \\
\hline 10 & Lithostrat unit & Symbol & UE-25 A\#1 & type & UE-25 A\#4 \\
\hline 11 & Not described & NC & 0.0 & & 0.0 \\
\hline 12 & alluvium & Qa & 0.0 & & 0.0 \\
\hline 13 & Rainier Mesa Tuff, includes pre-Rainier Mesa Tuff bedded tuff & Tmr & 30.0 & & 30.0 \\
\hline 14 & thyolite of Comb Peak & Tpk & 30.0 & & 30.0 \\
\hline 15 & Tiva Canyon Tuff (Tpc) nondivided & Tpc_un & 30.0 & & 30.0 \\
\hline 16 & Tpc, crystal-poor vitric densely welded subzone & Tpcpv3 & 196.0 & & 119.0 \\
\hline 17 & Tpc, crystal-poor vitric moderately welded subzone & Tpсpv2 & 196.0 & & 119.0 \\
\hline 18 & Tpc, crystal-poor vitric nonwelded to partially welded subzones & Tpcpvi & 205.0 & & 135.0 \\
\hline 19 & pre-Tiva Canyon Tuff bedded tuff & Tpbt4 & 210.0 & & 150.7 \\
\hline 20 & Yucca Mountain Tuff nondivided & Tpy & 217.3 & & 154.3 \\
\hline 21 & pre-Yucca Mountain Tuff bedded tuff & Tpbt3 & 217.3 & & $\mathbf{1 7 9 . 2}$ \\
\hline 22 & Pah Canyon Tuff nondivided & Tpp & 218.2 & & 197.0 \\
\hline 23 & pre-Pah Canyon Tuff bedded tuff & Tpbt2 & 245.9 & & 273.6 \\
\hline 24 & Topopah Spring Tuff (Tpt) crystal-rich vitric nonwelded to partially welded zones & Tptrv3 & 266.8 & & 301.9 \\
\hline 25 & Tpt, crystal-rich vitric moderately welded zone & Tptrv2 & 273.0 & & 309.0 \\
\hline 26 & Tpt, crystal-rich vitric densely welded zone & Tptrv1 & 275.6 & & 316.8 \\
\hline 27 & Tpt, crystal-rich nonlithophysal zone & Tptm & 279.5 & & 317.0 \\
\hline 28 & Tpt crystal-rich lithophysal zone & Tptri & 409.8 & & \\
\hline 29 & Tpt, lithic-rich zone & Tptf & 438.0 & & \\
\hline 30 & Tpt, crystal-poor upper lithophysal zone & Tptpul & 438.0 & & \\
\hline 31 & Tpt, crystal-poor middle nonlithophysal zone & Tptpmn & 673.0 & & \\
\hline 32 & Tpt, crystal-poor lower lithophysal zone & Tptpll & 745.0 & & \\
\hline 33 & Tpt, crystal-poor lower nonlithophysal zone & Tptpln & 1084.0 & & \\
\hline 34 & Tpt, crystal-poor vitric densely welded subzone & Tptpv3 & 1271.6 & & \\
\hline 35 & Tpt, crystal-poor vitric moderately welded subzone & Tptpv2 & 1310.1 & & \\
\hline 36 & Tpt, crystal-poor vitric nonwelded to partially welded subzones & Tptpv1 & 1324.6 & & \\
\hline 37 & pre-Topopah Spring Tuff bedded tuff & Tpbtl & 1360.0 & & \\
\hline 38 & Calico Hills Formation undifferentiated & Tac & 1368.6 & & \\
\hline 39 & pre-Calico Hills Formation bedded tuff & Tacbt & 1789.3 & & \\
\hline 40 & Prow Pass Tuff (Tcp) upper vitric(zeolitic) nonwelded to partially welded zones & Tcpuv & 1832.2 & & \\
\hline 41 & Tep, upper crystallized nonwelded to partially welded zones & Tcpuc & 1845.1 & & \\
\hline 42 & Tcp, crystallized moderately to densely welded zones & Tcpm & 1944.0 & & \\
\hline 43 & Tcp, lower crystallized nonwelded to partially welded zones & Tcplc & 2006.0 & & \\
\hline 44 & Tcp, lower vitric(zeolitic) nonwelded to partially welded zones & Teplv & 2030.0 & & \\
\hline 45 & pre-Prow Pass Tuff bedded tuff & Tepbt & 2331.4 & & \\
\hline 46 & Bullfrog Tuff (Tcb) upper vitric(zeolitic) nonwelded to partially welded zones & Tcbuv & 2333.2 & & \\
\hline 47 & Tcb, upper crystallized nonwelded to partially welded zones & Tcbuc & 2333.2 & & \\
\hline 48 & Tcb, crystallized moderately to densely welded zones & Tcbm & 2415.0 & & \\
\hline 49 & Tcb, lower crystallized nonwelded to partially welded zones & Tcble & & & \\
\hline 50 & Tcb, lower vitric(zeolitic) nonwelded to partially welded zones & Tcblv & & & \\
\hline 51 & pre-Bullfrog Tuff bedded tuff & Tcbbt & & & \\
\hline 52 & Tram Tuff (Tct) upper vitric(zeolitic) nonwelded to partially welded zones & Tetuv & & & \\
\hline 53 & Tet, upper crystallized nonwelded to partially welded zones & Tetuc & & & \\
\hline 54 & Tet, crystallized moderately to densely welded zones & Tetm & & & \\
\hline 55 & Tct, lower crystallized nonwelded to partially welded zones & Tetle & & & \\
\hline 56 & Tct, lower vitric(zeolitic) nonwelded to partially welded zones & Tetlv & & & \\
\hline 57 & pre-Tram Tuff bedded tuff & Tctbt & & & \\
\hline 58 & Lower Tertiary units undifferentiated & Tund & & & \\
\hline 59 & Palezoic and older units & $\mathbf{P z}$ & & & \\
\hline 60 & Vitric-Zeolitc boundary (noncrystallized rocks pervasively vitric versus zeolitic) & $\mathrm{V}-\mathrm{Z}$ & 1360.0 & & \\
\hline 61 & Repository Host Horizon (top) & RHH & 639.0 & & \\
\hline
\end{tabular}




\begin{tabular}{|c|c|c|c|c|c|c|c|c|c|}
\hline & $\mathbf{F}$ & G & $\mathrm{H}$ & I & $\mathbf{J}$ & $\mathbf{K}$ & $\mathrm{L}$ & M & $\mathbf{N}$ \\
\hline 1 & Clayton. & & & & & & & & \\
\hline 2 & & & & & & & & & \\
\hline 3 & \multicolumn{9}{|c|}{ llenged by overview committee during the January 21-22, 1998, workshop. } \\
\hline 4 & \multicolumn{9}{|c|}{ stions by Clayton after review workshop in addition to re-examination in the context of committee challenges. } \\
\hline 5 & & & & & & & & & \\
\hline 6 & & & & & & & & & \\
\hline 7 & & & & & & & & & \\
\hline 8 & ? & & & & & & & & \\
\hline 9 & Contact & & Contact & & Contact & & Contact & & Contact \\
\hline $\begin{array}{l}10 \\
11\end{array}$ & troe & UE-25 A\#5 & tope & UE-25A\#6 & type & UE-25 A\#7 & type & UE-25 B\#1 & type \\
\hline 12 & & $\begin{array}{r}0.0 \\
0.0\end{array}$ & & $\begin{array}{r}0.0 \\
0.0\end{array}$ & & \begin{tabular}{r|}
0.0 \\
0.0
\end{tabular} & & $\begin{array}{r}0.0 \\
0.0\end{array}$ & \\
\hline 13 & & 90.0 & & 20.0 & & 165.0 & & 156.0 & \\
\hline 14 & & 90.0 & & 20.0 & & 165.0 & & 156.0 & \\
\hline 15 & & 90.0 & & 20.0 & & 165.0 & & 156.0 & \\
\hline 16 & & 128.0 & & 124.5 & & 170.0 & & 180.0 & \\
\hline 17 & & 128.0 & & 124.5 & & 170.0 & & 180.0 & \\
\hline 18 & & 136.0 & & 135.0 & & 175.8 & & 182.0 & \\
\hline 19 & & 149.0 & & 144.2 & & 190.0 & & 189.0 & \\
\hline 20 & & 155.0 & & 149.3 & & 194.2 & & 192.5 & \\
\hline 21 & & 164.5 & & 167.0 & & 212.0 & & 192.5 & \\
\hline 22 & & 180.0 & & 186.0 & & 226.5 & & 204.5 & \\
\hline 23 & & 233.0 & & 201.5 & & 266.7 & & 243.0 & \\
\hline 24 & & 262.0 & & 229.8 & & 291.9 & & 259.0 & \\
\hline 25 & & 269.0 & & 236.0 & & 303.6 & & 267.0 & \\
\hline 26 & & 277.0 & & 241.7 & & 304.5 & & 275.0 & \\
\hline 27 & & 277.0 & & 242.0 & & 311.0 & & 279.9 & \\
\hline 28 & & 442.4 & & 402.0 & & 483.0 & & 413.0 & \\
\hline 29 & & 475.0 & & 422.0 & & 508.0 & & 440.0 & \\
\hline 30 & & 475.0 & & 422.0 & & 508.0 & & 440.0 & \\
\hline 31 & & & & & & 770.0 & & 680.5 & \\
\hline 32 & & & & & & 878.8 & & 765.0 & \\
\hline 33 & & & & & & & & 1130.0 & \\
\hline 34 & & & & & & & & 1283.0 & \\
\hline 35 & & & & & & & & 1336.0 & \\
\hline 36 & & & & & & & & 1352.0 & \\
\hline 37 & & & & & & & & 1374.0 & \\
\hline 38 & & & & & & & & 1385.0 & \\
\hline 39 & & & & & & & & 1845.0 & \\
\hline 40 & & & & & & & & 1882.0 & \\
\hline 41 & & & & & & & & 1896.0 & \\
\hline 42 & & & & & & & & 1992.0 & \\
\hline 43 & & & & & & & & 2039.0 & \\
\hline 44 & & & & & & & & 2071.0 & \\
\hline 45 & & & & & & & & 2355.6 & \\
\hline 46 & & & & & & & & 2361.3 & \\
\hline 47 & & & & & & & & 2361.3 & \\
\hline 48 & & & & & & & & 2468.0 & \\
\hline 49 & & & & & & & & 2782.8 & \\
\hline 50 & & & & & & & & 2799.5 & \\
\hline 51 & & & & & & & & 2852.7 & \\
\hline 52 & & & & & & & & 2882.5 & \\
\hline 53 & & & & & & & & 2933.0 & \\
\hline 54 & & & & & & & & 3158.0 & \\
\hline 55 & & & & & & & & 3322.0 & \\
\hline 56 & & & & & & & & 3359.9 & \\
\hline 57 & & & & & & & & 3900.9 & \\
\hline 58 & & & & & & & & 3960.3 & \\
\hline 59 & & & & & & & & & \\
\hline 60 & & & & & & & & 1336.0 & \\
\hline 61 & & & & & & & & 632.0 & \\
\hline
\end{tabular}




\begin{tabular}{|c|c|c|c|c|c|c|c|c|c|}
\hline & $\overline{0}$ & $\mathbf{P}$ & $Q$ & $\mathbf{R}$ & $\mathrm{S}$ & $\mathrm{T}$ & $\mathrm{U}$ & $\mathrm{V}$ & $\mathrm{W}$ \\
\hline 1 & & & & & & & & & \\
\hline 2 & & & & & & & & & \\
\hline 3 & . & & & & & & & & \\
\hline 4 & & & & & & & & & \\
\hline 5 & & & & & & & & & \\
\hline 6 & & & & & & & & & \\
\hline 7 & & & & & & & & & \\
\hline 8 & & & & & & & & & \\
\hline 9 & & Contact & & Contact & & Contact & & Contact & \\
\hline 10 & UE-25 C\#L & tyoe & UE $-25 \mathrm{CH} 2$ & tyoe & UE-25 C\#3 & type & USW G-1 & type & USW G-2 \\
\hline 11 & 0.0 & & 0.0 & & 0.0 & & 0.0 & & 0.0 \\
\hline 12 & 0.0 & & 0.0 & & 0.0 & & 0.0 & & 0.0 \\
\hline 13 & 0.0 & & 69.9 & & 80.1 & & 60.0 & & 0.0 \\
\hline 14 & 0.0 & & 69.9 & & 80.1 & & 60.0 & & 0.0 \\
\hline 15 & 60.0 & & 69.9 & & 80.1 & & 60.0 & & 0.0 \\
\hline 16 & 251.0 & & 243.0 & & 221.0 & & 60.0 & & 225.0 \\
\hline 17 & 251.0 & & 243.0 & & 221.0 & & 60.0 & & 225.0 \\
\hline 18 & 266.0 & & 257.0 & & 238.0 & & 60.0 & & 228.0 \\
\hline 19 & 271.0 & & 264.0 & & 247.0 & & 60.0 & & 235.0 \\
\hline 20 & 274.0 & & 267.0 & & 250.0 & & 60.0 & & 245.0 \\
\hline 21 & 274.0 & & 267.0 & & 250.0 & & 102.0 & & 341.5 \\
\hline 22 & 300.0 & & 286.0 & & 271.0 & & 135.0 & & 494.2 \\
\hline 23 & 300.0 & & 286.0 & & 271.0 & & 235.0 & & 730.8 \\
\hline 24 & 319.0 & & 306.0 & & 286.0 & & 265.0 & & 755.2 \\
\hline 25 & 327.0 & & 313.0 & & 295.0 & & 265.0 & & 761.7 \\
\hline 26 & 329.0 & & 315.0 & & 298.0 & & 270.0 & & 766.8 \\
\hline 27 & 332.0 & & 318.0 & & 303.0 & & 280.0 & & 771.2 \\
\hline 28 & 424.0 & & 423.0 & & 400.0 & & 438.0 & & 909.1 \\
\hline 29 & 438.0 & & 457.0 & & 438.0 & & 456.5 & & 909.1 \\
\hline 30 & 438.0 & & 457.0 & & 438.0 & & 456.5 & & 977.2 \\
\hline 31 & 595.0 & & 591.0 & & 563.0 & & 713.4 & & 1246.0 \\
\hline 32 & 726.0 & & 725.0 & & 703.0 & & 814.8 & & 1280.0 \\
\hline 33 & 1040.0 & & 1038.0 & & 1030.0 & & 1199.2 & & 1604.0 \\
\hline 34 & 1216.0 & & 1205.0 & & 1183.0 & & 1287.0 & & 1633.8 \\
\hline 35 & 1293.0 & & 1290.0 & & 1270.0 & & 1342.4 & & 1670.0 \\
\hline 36 & 1320.0 & & 1320.0 & & 1298.0 & & 1360.5 & & 1684.5 \\
\hline 37 & 1334.0 & & 1335.0 & & 1320.0 & & 1403.9 & & 1702.1 \\
\hline 38 & 1334.0 & & 1335.0 & & 1320.0 & & 1425.5 & & 1757.0 \\
\hline 39 & 1581.0 & & 1580.0 & & 1580.0 & & 1736.4 & & 2576.7 \\
\hline 40 & 1692.0 & & 1658.0 & & 1635.0 & & 1799.0 & & 2704.7 \\
\hline 41 & 1692.0 & & 1658.0 & & 1635.0 & & 1862.5 & & 2704.7 \\
\hline 42 & 1787.0 & & 1773.0 & & 1762.0 & & 1920.0 & & 2704.7 \\
\hline 43 & 1863.0 & & 1849.0 & & 1838.0 & & 1960.0 & & 2963.7 \\
\hline 44 & 1884.0 & & 1872.0 & & 1863.0 & & 1985.7 & & 2980.0 \\
\hline 45 & 2119.0 & & 2109.5 & & 2110.0 & & 2154.9 & & 3246.5 \\
\hline 46 & 2153.0 & & 2138.0 & & 2130.0 & & 2173.2 & & 3281.9 \\
\hline 47 & 2240.0 & & 2227.0 & & 2218.0 & & 2337.0 & & 3302.5 \\
\hline 48 & 2275.0 & & 2262.0 & & 2267.0 & & 2461.0 & & 3320.0 \\
\hline 49 & 2446.0 & & 2445.0 & & 2428.0 & & 2547.0 & & 3447.0 \\
\hline 50 & 2575.0 & & 2550.0 & & 2547.0 & & 2547.0 & & 3485.0 \\
\hline 51 & 2692.0 & & 2667.0 & & 2670.0 & & 2601.6 & & 3503.4 \\
\hline 52 & 2754.0 & & 2725.0 & & 2704.0 & & 2639.4 & & 3574,0 \\
\hline 53 & 2754.0 & & 2725.0 & & 2704.0 & & 2800.0 & & 3574.0 \\
\hline 54 & & & & & & & 2840.0 & & 3574.0 \\
\hline 55 & & & & & & & 2956.0 & & 3574.0 \\
\hline 56 & & & & & & & 3005.0 & & 3574.0 \\
\hline 57 & & & & & & & 3522.0 & & 3914.0 \\
\hline 58 & & & & & & & 3558.2 & & 3982.0 \\
\hline 59 & & & & & & & & & \\
\hline 60 & 1293.0 & & 1290.0 & & 1270.0 & & 1394.3 & & 1670.0 \\
\hline 61 & 515.0 & & 520.0 & & 500 & & 600.0 & & 1131.9 \\
\hline
\end{tabular}




\begin{tabular}{|c|c|c|c|c|c|c|c|c|c|}
\hline & $\mathrm{X}$ & $Y$ & $Z$ & AA & $\mathrm{AB}$ & $\mathrm{AC}$ & $\mathrm{AD}$ & $\mathrm{AE}$ & $\mathrm{AF}$ \\
\hline 1 & & & & & & & & & \\
\hline 2 & & & & & & & & & \\
\hline 3 & & & & & & & & & \\
\hline 4 & & & & & & & & & \\
\hline 5 & & & & & & & & & \\
\hline 6 & & & & & & & & & \\
\hline 7 & & & & & & & & & \\
\hline 8 & & & & & & & & & \\
\hline 9 & Contact & & Contact & & Contact & & Contact & & Contact \\
\hline 10 & type & USW GUU-3/G-3 & tupe & USW G-4 & type & USW H-1 & type & USW H-3 t & type \\
\hline 11 & & 0.0 & & 0.0 & & 0.0 & & 0.0 & \\
\hline 12 & & 0.0 & & 0.0 & & 0.0 & & 0.0 & \\
\hline 13 & & 0.0 & & 30.0 & & 0.0 & & 0.0 & \\
\hline 14 & & 0.0 & & 30.0 & & 0.0 & & 0.0 & \\
\hline 15 & & 0.0 & & 30.0 & & 0.0 & & $\begin{array}{l}0.0 \\
0.0\end{array}$ & \\
\hline 16 & & 348.1 & & 118.0 & & 61.0 & & 369.8 & \\
\hline 17 & & 357.0 & & 118.0 & & 61.0 & & 376.0 & \\
\hline 18 & & 368.4 & & 130.0 & & 70.0 & & 388.0 & \\
\hline 19 & & 372.5 & & 141.0 & & 90.0 & & 400.0 & \\
\hline 20 & & 375.5 & & 146.0 & & 95.1 & & 403.9 & \\
\hline 21 & & 375.5 & & 148.8 & & 160.8 & & 403.9 & \\
\hline 22 & & 391.7 & & 168.2 & & 190.3 & & 417.0 & \\
\hline 23 & & 391.7 & & 198.9 & & 278.9 & & 417.0 & \\
\hline 24 & & 417.7 & & 224.0 & & 295.0 & & 435.0 & \\
\hline 25 & & 424.9 & & 236.5 & & 320.0 & & 441.9 & \\
\hline 26 & & 427.8 & & 239.0 & & 330.0 & & 445.0 & \\
\hline 27 & & 427.8 & & 242.8 & & 335.0 & & 449.0 & \\
\hline 28 & & 542.0 & & 400.4 & & 505.0 & & 526.9 & \\
\hline 29 & & 548.0 & & 420.0 & & 538.0 & & \begin{tabular}{l|l|}
540.0 \\
\end{tabular} & \\
\hline 30 & & 548.0 & & 420.0 & & 538.0 & & 540.0 & \\
\hline 31 & & 688.0 & & 674.0 & & 788.0 & & 680.1 & \\
\hline 32 & & 830.0 & & 774.0 & & 897.0 & & 848.1 & \\
\hline 33 & & 1044.0 & & 1127.9 & & 1324.0 & & 1049.9 & \\
\hline 34 & & 1186.7 & & 1316.5 & & 1410.0 & & 1194.0 & \\
\hline 35 & & 1280.0 & & 1345.4 & & 1469.5 & & 1308.0 & \\
\hline 36 & & 1317.0 & & 1353.6 & & 1486.2 & & 1341.0 & \\
\hline 37 & & 1406.3 & & 1406.8 & & 1498.0 & & 1392.0 & \\
\hline 38 & & 1412.5 & & 1409.4 & & 1505.0 & & 1400.0 & \\
\hline 39 & & 1506.3 & & 1705.4 & & 1802.0 & & 1437.0 & \\
\hline 40 & & 1553.9 & & 1762.7 & & 1861.0 & & 1495.0 & \\
\hline 41 & & 1597.0 & & 1793.6 & & 1911.0 & & 1518.0 & \\
\hline 42 & & 1663.0 & & 1880.0 & & 1969.0 & & 1640.0 & \\
\hline 43 & & 1744.0 & & 1946.0 & & 2021.0 & & 1690.0 & \\
\hline 44 & & 1755.0 & & 1954.6 & & 2053.0 & & 1702.0 & \\
\hline 45 & & 1992.3 & & 2238.0 & & 2300.0 & & 1899.9 & \\
\hline 46 & & 1998.7 & & 2245.7 & & 2319.5 & & 1907.1 & \\
\hline 47 & & 2021.3 & & 2255.0 & & 2337.0 & & 1922.0 & \\
\hline 48 & & 2102.0 & & 2560.0 & & 2533.0 & & 2092.0 & \\
\hline 49 & & 2549.5 & & 2677.0 & & 2629.0 & & 2350.0 & \\
\hline 50 & & 2550.8 & & 2677.0 & & 2676.0 & & 2397.0 & \\
\hline 51 & & 2617.0 & & 2733.3 & & 2690.3 & & 2449.1 & \\
\hline 52 & & 2637.0 & & 2755.6 & & 2729.6 & & 2477.0 & \\
\hline 53 & & 2719.0 & & 2839.0 & & 2823.0 & & 2567.0 & \\
\hline 54. & & 2890.0 & & 2950.0 & & 2862.0 & & 2692.0 & \\
\hline 55 & & 3265.0 & & & & 3073.0 & & 3086.0 & \\
\hline 56 & & 3290.0 & & & & 3111.0 & & 3120.0 & \\
\hline 57 & & 3850.1 & & & & 3618.7 & & 3595.1 & \\
\hline 58 & & 3876.3 & & & & 3661.4 & & 3637.1 & \\
\hline 59 & & & & & & & & & \\
\hline 60 & & 1816.0 & & 1376.0 & & 1490.0 & & 1762.0 & \\
\hline 61 & & $\begin{array}{l}675.0 \\
\end{array}$ & & 618.0 & & 650.0 & & 605.0 & \\
\hline
\end{tabular}




\begin{tabular}{|c|c|c|c|c|c|c|c|c|c|}
\hline \multirow{2}{*}{1} & $\mathrm{AG}$ & $\mathbf{A H}$ & $\overline{\mathrm{AI}}$ & $\overline{\mathrm{AJ}}$ & $\mathrm{AK}$ & $\overline{\mathrm{AL}}$ & $\mathrm{AM}$ & $\mathrm{AN}$ & $\mathrm{AO}$ \\
\hline & & & & & & & & & \\
\hline \multicolumn{10}{|l|}{2} \\
\hline \multicolumn{10}{|l|}{3} \\
\hline \multicolumn{10}{|l|}{4} \\
\hline \multicolumn{10}{|l|}{5} \\
\hline \multicolumn{10}{|l|}{6} \\
\hline \multicolumn{10}{|l|}{7} \\
\hline \multicolumn{10}{|l|}{8} \\
\hline 9 & & Contact & & Contact & & Contact & & Contact & \\
\hline 10 & USWH-4 & tope & USWH-5 & tope & USW H-6 & type & UE-25 J\#13 & type & UE-25 NRG\#1 \\
\hline 11 & $\begin{array}{r}0.0 \\
\end{array}$ & & $\begin{array}{r}0.0 \\
\end{array}$ & & 0.0 & & $\begin{array}{r} \\
0.0 \\
\end{array}$ & & $\begin{array}{r}0.0 \\
\end{array}$ \\
\hline 12 & 0.0 & & 0.0 & & 0.0 & & 0.0 & & 9.5 \\
\hline 13 & 0.0 & & 0.0 & & 29.9 & & 435.0 & & 9.5 \\
\hline 14 & 0.0 & & 0.0 & & 29.9 & & 435.0 & & 9.5 \\
\hline 15 & 0.0 & & 0.0 & & 29.9 & & 435.0 & & 9.5 \\
\hline 16 & 173.9 & & 404.0 & & 190.0 & & 587.0 & & \\
\hline 17 & 173.9 & & 404.0 & & 190.0 & & 591.0 & & \\
\hline 18 & 185.0 & & 420.0 & & 200.1 & & 610.0 & & \\
\hline 19 & 193.0 & & 437.5 & & 260.2 & & 629.0 & & \\
\hline 20 & 195.0 & & 438.0 & & 270.0 & & 632.0 & & \\
\hline 21 & 198.0 & & 457.0 & & 275.0 & & 632.0 & & \\
\hline 22 & 216.0 & & 471.0 & & 278.0 & & 650.0 & & \\
\hline 23 & 224.0 & & 510.0 & & 290.0 & & 650.0 & & \\
\hline 24 & 242.0 & & 542.0 & & 300.2 & & 682.0 & & \\
\hline 25 & 248.5 & & 560.0 & & 330.0 & & 686.0 & & \\
\hline 26 & 251.0 & & 562.0 & & 330.0 & & 691.0 & & \\
\hline 27 & 254.0 & & 564.0 & & 332.0 & & 691.0 & & \\
\hline 28 & 376.0 & & 700.0 & & 409.0 & & 755.0 & & \\
\hline 29 & 376.0 & & 741.0 & & 435.0 & & 801.0 & & \\
\hline 30 & 376.0 & & 741.0 & & 435.0 & & 801.0 & & \\
\hline 31 & 576.0 & & 988.0 & & 653.0 & & 905.0 & & \\
\hline 32 & 703.0 & & 1088.0 & & 795.0 & & 1003.0 & & \\
\hline 33 & 987.0 & & 1450.0 & & 1097.0 & & 1193.0 & & \\
\hline 34 & 1185.0 & & 1582.0 & & 1213.0 & & 1300.0 & & \\
\hline 35 & 1209.0 & & 1659.0 & & 1310.0 & & 1415.0 & & \\
\hline 36 & 1247.0 & & 1672.0 & & 1322.0 & & 1450.0 & & \\
\hline 37 & 1312.0 & & 1699.1 & & 1356.0 & & 1475.0 & & \\
\hline 38 & 1317.0 & & 1705.0 & & 1356.0 & & 1482.0 & & \\
\hline 39 & 1572.0 & & 1879.9 & & 1458.0 & & 1682.0 & & \\
\hline 40 & 1626.9 & & 1944.9 & & 1508.0 & & 1711.0 & & \\
\hline 41 & 1662.0 & & 1967.0 & & 1555.0 & & 1742.0 & & \\
\hline 42 & 1746.0 & & 2085.0 & & 1602.0 & & 1848.0 & & \\
\hline 43 & 1820.0 & & 2113.0 & & 1670.0 & & 1942.0 & & \\
\hline 44 & 1840.0 & & 2130.0 & & 1685.0 & & 1961.0 & & \\
\hline 45 & 2263.1 & & 2240.1 & & 1765.1 & & 1993.0 & & \\
\hline 46 & 2274.9 & & 2263.1 & & 1794.9 & & 2017.0 & & \\
\hline 47 & 2369.0 & & 2310.0 & & 1881.0 & & 2017.0 & & \\
\hline 48 & 2494.0 & & 2388.0 & & 1894.0 & & 2082.0 & & \\
\hline 49 & 2559.0 & & 2468.0 & & 1990.0 & & 2322.0 & & \\
\hline 50 & 2635.0 & & 2510.0 & & 2138.1 & & 2322.0 & & \\
\hline 51 & 2644.0 & & 2712.9 & & 2225.0 & & 2322.0 & & \\
\hline 52 & 2664.0 & & 2742.1 & & 2258.0 & & 2358.0 & & \\
\hline 53 & 2745.0 & & 2845.0 & & 2348.0 & & 2465.0 & & \\
\hline 54 & 2835.0 & & 2897.0 & & 2439.0 & & 2658.0 & & \\
\hline 55 & 3200.0 & & 3130.0 & & 2655.0 & & 2862.0 & & \\
\hline 56 & 3228.0 & & 3150.0 & & 2667.0 & & 2991.0 & & \\
\hline 57 & 3788.0 & & 3412.0 & & 2869.1 & & 3200.0 & & \\
\hline 58 & 3818.9 & & 3421.9 & & 2877.9 & & 3220.0 & & \\
\hline 59 & & & & & & & & & \\
\hline 60 & 1330.0 & & 1888.0 & & 1429.0 & & 1415.0 & & \\
\hline 61 & 553.0 & & 931.0 & & 585.0 & & & & \\
\hline
\end{tabular}




\begin{tabular}{|c|c|c|c|c|c|c|c|}
\hline & $\widehat{\mathrm{AP}}$ & $\mathrm{AQ}$ & $\overline{\mathrm{AR}}$ & $A S$ & AT & $\mathrm{AU}$ & $\overline{\mathrm{AV}}$ \\
\hline \multirow{2}{*}{\multicolumn{8}{|c|}{$\frac{1}{2}$}} \\
\hline & & & & & & & \\
\hline \multicolumn{8}{|l|}{3} \\
\hline \multicolumn{8}{|l|}{4} \\
\hline \multicolumn{8}{|l|}{$\frac{5}{6}$} \\
\hline \multirow{2}{*}{\multicolumn{8}{|c|}{7}} \\
\hline & & & & & & & \\
\hline \multicolumn{8}{|l|}{8} \\
\hline 9 & Contact & & Contact & & Contact & & Contact \\
\hline 10 & tyoe & UE-25 NRG\#2 & tope & UE-25 NRG\#2A & type & UE-25 NRG\#2B & type \\
\hline 11 & & 0.0 & & $\begin{array}{r}0.0 \\
\end{array}$ & & \begin{tabular}{|r|} 
\\
0.0 \\
\end{tabular} & \\
\hline 12 & & 0.0 & & 80.6 & & 0.0 & \\
\hline 13 & & 0.0 & & 80.6 & & 2.2 & \\
\hline 14 & & 164.6 & & 80.6 & & 157.3 & \\
\hline 15 & & 164.6 & & 165.9 & & 232.3 & \\
\hline 16 & & 276.3 & & & & 232.3 & \\
\hline 17 & & 276.3 & & & & 232.3 & \\
\hline 18 & & 282.8 & & & & 232.3 & \\
\hline 19 & & & & & & 265.2 & \\
\hline 20 & & & & & & 267.1 & \\
\hline 21 & & & & & & 268.9 & \\
\hline 22 & & & & & & 285.3 & \\
\hline 23 & & & & & & 324.0 & \\
\hline \multirow{2}{*}{\multicolumn{8}{|c|}{$\frac{24}{25}$}} \\
\hline & & & & & & & \\
\hline \multicolumn{8}{|l|}{26} \\
\hline \multicolumn{8}{|l|}{27} \\
\hline \multirow{2}{*}{\multicolumn{8}{|c|}{$\frac{28}{29}$}} \\
\hline & & & & & & & \\
\hline \multicolumn{8}{|l|}{30} \\
\hline \multicolumn{8}{|l|}{31} \\
\hline \multirow{2}{*}{\multicolumn{8}{|c|}{$\frac{32}{33}$}} \\
\hline & & & & & & & \\
\hline \multicolumn{8}{|l|}{34} \\
\hline 35 & & & & & & & \\
\hline 36 & & & & & & & \\
\hline \begin{tabular}{|l|}
37 \\
\end{tabular} & & & & & & & \\
\hline 38 & & & & & & & \\
\hline \begin{tabular}{|l|}
39 \\
\end{tabular} & & & & & & & \\
\hline 40 & & & & & & & \\
\hline 41 & & & & & & & \\
\hline 42 & & & & & & & \\
\hline \begin{tabular}{|l|}
43 \\
\end{tabular} & & & & & & & \\
\hline \begin{tabular}{|l|}
44 \\
\end{tabular} & & & & & & & \\
\hline 45 & & & & & & & \\
\hline 46 & & & & & & & \\
\hline 47 & & & & & & & \\
\hline 48 & & & & & & & \\
\hline \begin{tabular}{|l|}
49 \\
\end{tabular} & & & & & & & \\
\hline 50 & & & & & & & \\
\hline 51 & & & & & & & \\
\hline 52 & & & & & & & \\
\hline 53 & & & & & & & \\
\hline 54 & & & & & & & \\
\hline 55 & & & & & & & \\
\hline 56 & & & & & & & \\
\hline 57 & & & & & $\cdots$ & & \\
\hline 58 & & & & & & & \\
\hline \begin{tabular}{|l|l}
59 \\
\end{tabular} & & & & & & & \\
\hline 60 & & & & & & & \\
\hline \begin{tabular}{|l|}
61 \\
\end{tabular} & & & & & & & \\
\hline
\end{tabular}




\begin{tabular}{|c|c|c|c|c|}
\hline & $\mathrm{AW}$ & $\mathrm{AX}$ & $\mathrm{AY}$ & $\overline{\mathrm{AZ}}$ \\
\hline \multicolumn{5}{|l|}{1} \\
\hline \multicolumn{5}{|l|}{2} \\
\hline \multicolumn{5}{|l|}{3} \\
\hline \multicolumn{5}{|l|}{4} \\
\hline \multicolumn{5}{|l|}{5} \\
\hline \multicolumn{5}{|l|}{6} \\
\hline \multicolumn{5}{|l|}{7} \\
\hline \multicolumn{5}{|l|}{8} \\
\hline 9 & & Contact & & Contact \\
\hline 10 & UE-25NRG\#2C & type & UE-25 NRG\#2D & type \\
\hline 11 & 0.0 & & 0.0 & \\
\hline 12 & 50.0 & & 38.1 & \\
\hline 13 & 50.0 & & 38.1 & \\
\hline 14 & 150.1 & & 125.9 & \\
\hline \multicolumn{5}{|l|}{15} \\
\hline \multicolumn{5}{|l|}{16} \\
\hline \multicolumn{5}{|l|}{17} \\
\hline \multicolumn{5}{|l|}{18} \\
\hline \multicolumn{5}{|l|}{19} \\
\hline \multicolumn{5}{|l|}{20} \\
\hline \multicolumn{5}{|l|}{21} \\
\hline \multicolumn{5}{|l|}{22} \\
\hline 23 & & & & \\
\hline 24 & & & & \\
\hline 25 & & & & \\
\hline 26 & & & & \\
\hline 27 & & & & \\
\hline 28 & & & & \\
\hline 29 & & & & \\
\hline 30 & & & & \\
\hline 31 & & & & \\
\hline 32 & & & & \\
\hline 33 & & & & \\
\hline 34 & & & & \\
\hline 35 & & & & \\
\hline 36 & & & & \\
\hline 37 & & & & \\
\hline 38 & & & & \\
\hline 39 & & & & \\
\hline 40 & & & & \\
\hline 41 & & & & \\
\hline 42 & & & & \\
\hline 43 & & & & \\
\hline 44 & & & & \\
\hline 45 & & & & \\
\hline 46 & & & & \\
\hline 47 & & & & \\
\hline 48 & & & & \\
\hline 49 & & & & \\
\hline 50 & & & & \\
\hline 51 & & & & \\
\hline 52 & & & & \\
\hline 53 & & & & \\
\hline 54 & & & & \\
\hline 55 & & & & \\
\hline 56 & & & & \\
\hline 57 & & & & \\
\hline 58 & & & & \\
\hline 59 & & & & \\
\hline 60 & & & & \\
\hline 61 & & & & \\
\hline
\end{tabular}




\begin{tabular}{|c|c|c|c|c|c|c|c|c|}
\hline & BA & $\mathrm{BB}$ & $\mathrm{BC}$ & $\mathrm{BD}$ & $\mathrm{BE}$ & $\mathrm{BF}$ & $\mathrm{BG}$ & $\mathrm{BH}$ \\
\hline 1 & & & & & & & & \\
\hline 2 & & & & & & & & \\
\hline 3 & & & & & & & & \\
\hline 4 & & & & & & & & \\
\hline 5 & & & & & & & & \\
\hline 6 & & & & & & & & \\
\hline 7 & & & & & & & & \\
\hline 8 & & & & & & & & \\
\hline 9 & & & Contact & & Contact & & Contact & \\
\hline 10 & Symbol & UE-25 NRGH3 & type & UE-25 NRG\#4 & type & UE-25 NRG\#5 & type & USW NRG-6 \\
\hline 11 & $\mathrm{NC}$ & 0.0 & & 0.0 & & 0.0 & & 0.0 \\
\hline 12 & $\mathrm{Qa}$ & 0.0 & & 0.0 & & 0.0 & & 0.0 \\
\hline 13 & Tmr & 0.0 & & 0.0 & & 0.0 & & 0.0 \\
\hline 14 & Tpk & 0.0 & & 0.0 & & 0.0 & & 0.0 \\
\hline 15 & Tpc un & 0.0 & & 0.0 & & 0.0 & & 0.0 \\
\hline 16 & Tpcpv3 & & & 318.0 & & 140.0 & & 135.3 \\
\hline 17 & Tpcpv2 & & & 318.0 & & 140.0 & & 135.3 \\
\hline 18 & Tpcpvl & & & 323.0 & & 154.0 & & 151.8 \\
\hline 19 & Tpbt4 & & & 338.0 & & 163.0 & & 158.6 \\
\hline 20 & Tpy & & & 344.0 & & 170.0 & & 162.8 \\
\hline 21 & Tpbt3 & & & 354.0 & & 187.0 & & 162.8 \\
\hline 22 & Tpp & & & 375.0 & & 215.0 & & 174.9 \\
\hline 23 & Tpbt2 & & & 458.0 & & 288.0 & & 220.8 \\
\hline 24 & Tptrv3 & & & 477.0 & & 321.0 & & 244.7 \\
\hline 25 & Tptrv2 & & & 481.5 & & 327.0 & & 257.4 \\
\hline 26 & Tptrvl & & & 485.0 & 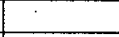 & 330.0 & & 259.8 \\
\hline 27 & Tptm & & & 488.9 & & 332.0 & & 263.2 \\
\hline 28 & Tptrl & & & 660.5 & & 517.0 & & 429.0 \\
\hline 29 & Tptf & & & 700.0 & & 565.0 & & 465.5 \\
\hline 30 & Tptpul & & & 700.0 & & 565.0 & & 465.5 \\
\hline 31 & Tptpmn & & & & & 770.0 & & 713.0 \\
\hline 32 & Tptpll & & & & & 901.5 & & 810.0 \\
\hline 33 & Tptpln & & & & & 1230.0 & & \\
\hline 34 & Tptpv3 & & & & & & & \\
\hline 35 & Tptpv2 & & & & & & & \\
\hline 36 & Tptpvl & & & & & & & \\
\hline 37 & Tpbt1 & & & & & & & \\
\hline 38 & Tac & & & & & & & \\
\hline 39 & Tacbt & & & & & & & \\
\hline 40 & Tcp4v & & & & & & & \\
\hline 41 & Tcp $3 n 2 c$ & & & & & & & \\
\hline 42 & Tcp3m-d & & & & & & & \\
\hline 43 & Tcp1-3n1c & & & & & & & \\
\hline 44 & Tcp1-3n1v & & & & & & & \\
\hline 45 & Tcpbt & & & & & & & \\
\hline 46 & Tcbn2v & & & & & & & \\
\hline 47 & Tcbn $2 \mathrm{c}$ & & & & & & & \\
\hline 48 & Tcbm-d & & & & & & & \\
\hline 49 & Tcbnlc & & & & & & & \\
\hline 50 & Tcbniv & & & & & & & \\
\hline 51 & Tcbbt & & & & & & & \\
\hline 52 & $\operatorname{Tctn} 2 \mathrm{v}$ & & & & & & & \\
\hline 53 & $\operatorname{Tctn} 2 c$ & & & & & & & \\
\hline 54 & Tctm-d & & & & & & & \\
\hline 55 & Tctnlc & & & & & & & \\
\hline 56 & Tetnlv & & & & & & & \\
\hline 57 & Tetbt & & & & & & & \\
\hline 58 & Tund & & & & & & & \\
\hline 59 & $\mathrm{Pz}$ & & & & & & & \\
\hline 60 & $V-Z$ & & & & & & & \\
\hline 61 & RHH & & & & & 681.0 & & 620.0 \\
\hline
\end{tabular}




\begin{tabular}{|c|c|c|c|c|c|c|c|c|c|}
\hline & BI & BJ & BK & $\overline{\mathrm{BL}}$ & BM & $\mathrm{BN}$ & BO & $\mathrm{BP}$ & $\overline{\mathrm{BQ}}$ \\
\hline 1 & & & & & & & & & \\
\hline 2 & & & & & & & & & \\
\hline 3 & & & & & & & & & \\
\hline 4 & & & & & & & & & \\
\hline 5 & & & & & & & & & \\
\hline 6 & & & & & & & & & \\
\hline 7 & & & & & & & & & \\
\hline 8 & & & & & & & & & \\
\hline 9 & Contact & & Contact & & Contact & & Contact & & Contact \\
\hline 10 & type & USW NRG-7/7A & tope & $\mathrm{ONC} \# 1$ & type & UE-25 P\#1 & type & USW SD-7 & type \\
\hline 11 & & 0.0 & & 0.0 & & 0.0 & & 0.0 & \\
\hline 12 & & 17.0 & & 0.0 & & 0.0 & & 50.1 & \\
\hline 13 & & 17.0 & & 98.0 & & 127.0 & & 50.1 & \\
\hline 14 & & 17.0 & & 193.0 & & 127.0 & & 50.1 & \\
\hline 15 & & 17.0 & & 206.0 & & 127.0 & & 50.1 & \\
\hline 16 & & 69.7 & & 578.0 & & 127.0 & & 305.0 & \\
\hline 17 & & 69.7 & & 578.0 & & 127.0 & & 305.0 & \\
\hline 18 & & 79.2 & . & 589.0 & & 127.0 & & 316.0 & \\
\hline 19 & & 102.0 & & 597.0 & & 127.0 & & 325.8 & \\
\hline 20 & & 106.4 & & 600.0 & & 127.0 & & 330.6 & \\
\hline 21 & & 156.0 & & 600.0 & & 127.0 & & 330.6 & \\
\hline 22 & & 172.0 & & 621.0 & & 127.0 & & 343.0 & \\
\hline 23 & & 258.8 & & 621.0 & & 127.0 & & 356.0 & \\
\hline 24 & & 284.3 & & 643.0 & & 140.0 & & 384.3 & \\
\hline 25 & & 292.7 & & 653.0 & & 145.0 & & 384.3 & \\
\hline 26 & & 296.2 & & 654.0 & & 148.0 & & 386.3 & \\
\hline 27 & & 299.0 & & 658.0 & & 150.0 & & 386.3 & \\
\hline 28 & & 478.2 & & 774.0 & & 228.0 & & 480.0 & \\
\hline 29 & & 518.4 & & 810.0 & & 248.0 & & 490.0 & \\
\hline 30 & & 518.4 & & 810.0 & & 248.0 & & 490.0 & \\
\hline 31 & & 740.0 & & 977.0 & & 493.0 & & 682.5 & \\
\hline 32 & & 877.6 & & 1100.0 & & 640.0 & & 803.3 & \\
\hline 33 & & 1243.0 & & 1178.0 & & 958.0 & & 1020.0 & \\
\hline 34 & & 1414.8 & & 1178.0 & & 1090.0 & & 1182.0 & \\
\hline 35 & & 1457.0 & & 1178.0 & & 1200.0 & & 1285.0 & \\
\hline 36 & . & 1474.6 & & 1213.0 & & 1243.0 & & 1308.0 & \\
\hline 37 & & 1493.0 & & 1253.0 & & 1270.0 & & 1395.4 & \\
\hline 38 & & 1498.0 & & 1274.0 & & 1270.0 & & 1405.6 & \\
\hline 39 & & & & & & 1390.0 & & 1567.2 & \\
\hline 40 & & & & & & 1441.0 & & 1621.5 & \\
\hline 41 & & & & & & 1468.0 & & 1646.5 & \\
\hline 42 & & & & & & 1535.0 & & 1765.0 & \\
\hline 43 & & & & & & 1630.0 & & 1832.0 & \\
\hline 44 & & & & & & 1680.0 & & 1872.0 & \\
\hline 45 & & & & & & 1790.0 & & 2167.8 & \\
\hline 46 & & & & & & 1826.0 & & 2183.9 & \\
\hline 47 & & & & & & 1826.0 & & 2183.9 & \\
\hline 48 & & & & & & 1953.0 & & 2183.9 & \\
\hline 49 & & & . & & & 2130.0 & & 2450.0 & \\
\hline 50 & & & & & & 2162.0 & & 2478.0 & \\
\hline 51 & & & & & & 2240.0 & & 2579.4 & \\
\hline 52 & & & & & & 2262.0 & & 2598.0 & \\
\hline 53 & & & & & & 2340.0 & & 2611.8 & \\
\hline 54 & & & & & & 2395.0 & & & \\
\hline 55 & & & & & & 2595.0 & & & \\
\hline 56 & & & & & & 2616.0 & & & \\
\hline 57 & & & & & & 2863.0 & & & \\
\hline 58 & & & & & & 2863.0 & & & \\
\hline 59 & & & & & & 4080.0 & & & \\
\hline 60 & & 1466.0 & & 1153.0 & & 1200.0 & & 1562.0 & \\
\hline 61 & & 659.0 & & 927.0 & & 453.0 & & 640.0 & \\
\hline
\end{tabular}




\begin{tabular}{|c|c|c|c|c|c|c|c|c|c|}
\hline & BR & BS & $\overline{B T}$ & $\mathrm{BU}$ & BV & $\mathrm{BW}$ & $\mathrm{BX}$ & BY & $\overline{B Z}$ \\
\hline \multicolumn{10}{|l|}{1} \\
\hline \multicolumn{10}{|l|}{2} \\
\hline \multicolumn{10}{|l|}{3} \\
\hline \multicolumn{10}{|l|}{4} \\
\hline \multicolumn{10}{|l|}{5} \\
\hline \multicolumn{10}{|l|}{6} \\
\hline \multicolumn{10}{|l|}{7} \\
\hline \multicolumn{10}{|l|}{8} \\
\hline 9 & & Contact & & Contact & & Contact & & Contact & \\
\hline 10 & USW SD-9 & type & USW SD-12 & type & USW UZ-1 & type & UE-25 UZ\#4 & type & UE-25 UZ\#5 \\
\hline 11 & 0.0 & & 0.0 & & 0.0 & & 0.0 & & 0.0 \\
\hline 12 & 53.6 & 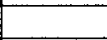 & 5.3 & & 0.0 & & 0.0 & & 3.0 \\
\hline 13 & 53.6 & & 5.3 & & 40.0 & & 39.0 & & 3.0 \\
\hline 14 & 53.6 & & 5.3 & & 40.0 & & 39.0 & & 3.0 \\
\hline 15 & 53.6 & & 5.3 & & 40.0 & & 39.0 & & 3.0 \\
\hline 16 & 57.2 & & 239.5 & & 40.0 & & 71.4 & & 89.0 \\
\hline 17 & 57.2 & & 239.5 & & 40.0 & & 71.4 & & 89.0 \\
\hline 18 & 76.5 & & 256.0 & & 40.0 & & 78.0 & & 100.0 \\
\hline 19 & 91.5 & & 263.7 & & 40.0 & & 99.0 & & 118.0 \\
\hline 20 & 95.9 & & 266.0 & & 40.0 & & 106.0 & & 122.0 \\
\hline 21 & 140.8 & & 266.0 & & 78.0 & & 151.5 & & 162.0 \\
\hline 22 & 155.5 & & 278.3 & & 105.0 & & 173.9 & & 186.0 \\
\hline 23 & 226.6 & & 291.2 & & 242.0 & & 305.0 & & 316.0 \\
\hline 24 & 255.6 & & 314.1 & & 272.0 & & 333.0 & & 345.0 \\
\hline 25 & 266.7 & & 320.8 & & 282.5 & & 343.0 & & 352.5 \\
\hline 26 & 268.5 & & 324.5 & & 284.0 & & 345.0 & & 354.5 \\
\hline 27 & 272.2 & & 330.7 & & 288.0 & & 346.0 & & 356.1 \\
\hline 28 & 450.0 & & 436.4 & & 436.0 & & & & \\
\hline 29 & 473.0 & & 470.2 & & 470.0 & & & & \\
\hline 30 & 473.0 & & 470.2 & & 470.0 & & & & \\
\hline 31 & 730.0 & & 663.7 & & 717.0 & & & & \\
\hline 32 & 845.8 & & 786.9 & & 830.0 & & & & \\
\hline 33 & 1182.0 & & 1065.5 & & 1145.0 & & & & \\
\hline 34 & 1358.0 & & 1278.1 & & & & & & \\
\hline 35 & 1418.4 & & 1308.0 & & & & & & \\
\hline 36 & 1425.7 & & 1337.5 & & & & & & \\
\hline 37 & 1464.1 & & 1408.1 & & & & & & \\
\hline 38 & 1479.9 & & 1411.5 & & & & & & \\
\hline 39 & 1764.4 & & 1599.5 & & & & & & \\
\hline 40 & 1820.7 & & 1648.4 & & & & & & \\
\hline 41 & 1868.7 & & 1677.0 & & & & & & \\
\hline 42 & 1938.5 & & 1787.0 & & & & & & \\
\hline 43 & 1991.4 & & 1842.0 & & & & & & \\
\hline 44 & 2015.8 & & 1865.0 & & & & & & \\
\hline 45 & & & 2133.0 & & & & & & \\
\hline 46 & & & 2137.8 & & & & & & \\
\hline 47 & & & & & & & & & \\
\hline 48 & & & & & & & & & \\
\hline 49 & & & & & & & & & \\
\hline 50 & & & & & & & & & \\
\hline 51 & & & & & & & & & \\
\hline 52 & & & & & & & & & \\
\hline 53 & & & & & & & & & \\
\hline 54 & & & & & & & & & \\
\hline 55 & & & & & & & & & \\
\hline 56 & & & & & & & & & \\
\hline 57 & & & & & & & & & \\
\hline 58 & & & & & & & & & \\
\hline 59 & & & & & & & & & \\
\hline 60 & 1457.0 & & 1600.0 & & & & & & \\
\hline 61 & 628.0 & & 630.0 & & 585.0 & & & & \\
\hline
\end{tabular}




\begin{tabular}{|c|c|c|c|c|c|c|c|c|c|}
\hline & $\mathrm{CA}$ & $\mathrm{CB}$ & $\mathrm{CC}$ & $\mathrm{CD}$ & $\mathrm{CE}$ & CF & CG & $\mathrm{CH}$ & $\mathrm{CI}$ \\
\hline \multicolumn{10}{|l|}{1} \\
\hline \multicolumn{10}{|l|}{2} \\
\hline \multicolumn{10}{|l|}{3} \\
\hline \multicolumn{10}{|l|}{4} \\
\hline \multicolumn{10}{|l|}{5} \\
\hline \multicolumn{10}{|l|}{6} \\
\hline \multicolumn{10}{|l|}{7} \\
\hline \multicolumn{10}{|l|}{8} \\
\hline 9 & Contact & & Contact & & Contact & & Contact & & Contact \\
\hline 10 & tope & USWUZ-6 & type & USWUZ-7A & type & USW UZ-14 & type & UE-25 UZ\#16 & type \\
\hline 11 & & 0.0 & & 0.0 & & \begin{tabular}{|r|}
0.0 \\
\end{tabular} & & 0.0 & \\
\hline 12 & & 0.0 & & 38.5 & & $\begin{array}{r}39.7 \\
\end{array}$ & & 0.0 & \\
\hline 13 & & 0.0 & & 38.5 & & 39.7 & & 39.7 & \\
\hline 14 & & 0.0 & & 38.5 & & 39.7 & & 39.7 & \\
\hline 15 & & 0.0 & & 38.5 & & 39.7 & & 39.7 & \\
\hline 16 & & 383.0 & & 163.9 & & 39.7 & & 140.8 & \\
\hline 17 & & 407.0 & & 163.9 & & 39.7 & & 140.8 & \\
\hline 18 & & 422.0 & & 184.0 & & 39.7 & & 153.0 & \\
\hline 19 & & 432.5 & & 197.7 & & 39.7 & & 160.7 & \\
\hline 20 & & 437.2 & & 203.6 & & 39.7 & & 165.9 & \\
\hline 21 & & 437.2 & & 203.6 & & 78.2 & & 173.4 & \\
\hline 22 & & 450.1 & & 214.9 & & 102.1 & & 188.8 & \\
\hline 23 & & 455.5 & & 218.6 & & 240.4 & & 188.8 & \\
\hline 24 & & 478.2 & & 243.0 & & 268.2 & & 217.0 & \\
\hline 25 & & 483.0 & & 247.3 & & 280.9 & & 228.1 & \\
\hline 26 & & 489.0 & & 248.6 & & 282.5 & & 229.4 & \\
\hline 27 & & 490.0 & & 248.6 & & 286.0 & & 238.9 & \\
\hline 28 & & 575.0 & & 377.8 & & 430.0 & & 357.8 & \\
\hline 29 & & 610.0 & & 377.8 & & 468.0 & & 371.0 & \\
\hline 30 & & 610.0 & & 377.8 & & 468.0 & & 371.0 & \\
\hline 31 & & 778.0 & & 480.0 & & 715.0 & & 545,0 & \\
\hline 32 & & 917.0 & & 607.0 & & 828.0 & & 669.0 & \\
\hline 33 & & 1190.0 & & & & 1138.0 & & 935.0 & \\
\hline 34 & & 1333.0 & & & & 1279.1 & & 1107.5 & \\
\hline 35 & & 1379.0 & & & & 1358.0 & & 1165.2 & \\
\hline 36 & & 1422.0 & & & & 1383.0 & & 1178.0 & \\
\hline 37 & & 1450.0 & & & & 1404.2 & & 1190.0 & \\
\hline 38 & & 1460.0 & & & & 1420.2 & & 1197.0 & \\
\hline 39 & & 1547.2 & & & & 1694.0 & & 1455.4 & \\
\hline 40 & & 1592.0 & & & & 1750.2 & & 1485.0 & \\
\hline 41 & & 1614.0 & & & & 1815.1 & & 1497.7 & \\
\hline 42 & & 1750.0 & & & & 1850.0 & & 1571.0 & \\
\hline 43 & & 1802.0 & & & & 1893.0 & & 1638.0 & \\
\hline 44 & & 1829.0 & & & & 1899.0 & & 1669.2 & \\
\hline 45 & & & & & & 2046.6 & & & \\
\hline 46 & & & & & & 2072.1 & & & \\
\hline \multicolumn{10}{|l|}{47} \\
\hline \multicolumn{10}{|l|}{48} \\
\hline \multicolumn{10}{|l|}{49} \\
\hline \multicolumn{10}{|l|}{50} \\
\hline \multicolumn{10}{|l|}{51} \\
\hline 52 & & & & & & & & & \\
\hline 53 & & & & & & & & & \\
\hline 54 & & & & & & & & & \\
\hline 55 & & & & & & & & & \\
\hline 56 & & & & & & & & & \\
\hline 57 & & & & & & & & & \\
\hline 58 & & & $\therefore$ & & & & & & \\
\hline 59 & & & & & & & & & \\
\hline 60 & & & & & & 1392.5 & & 1165.2 & \\
\hline 61 & & 690.0 & & & & & & 485.0 & \\
\hline
\end{tabular}




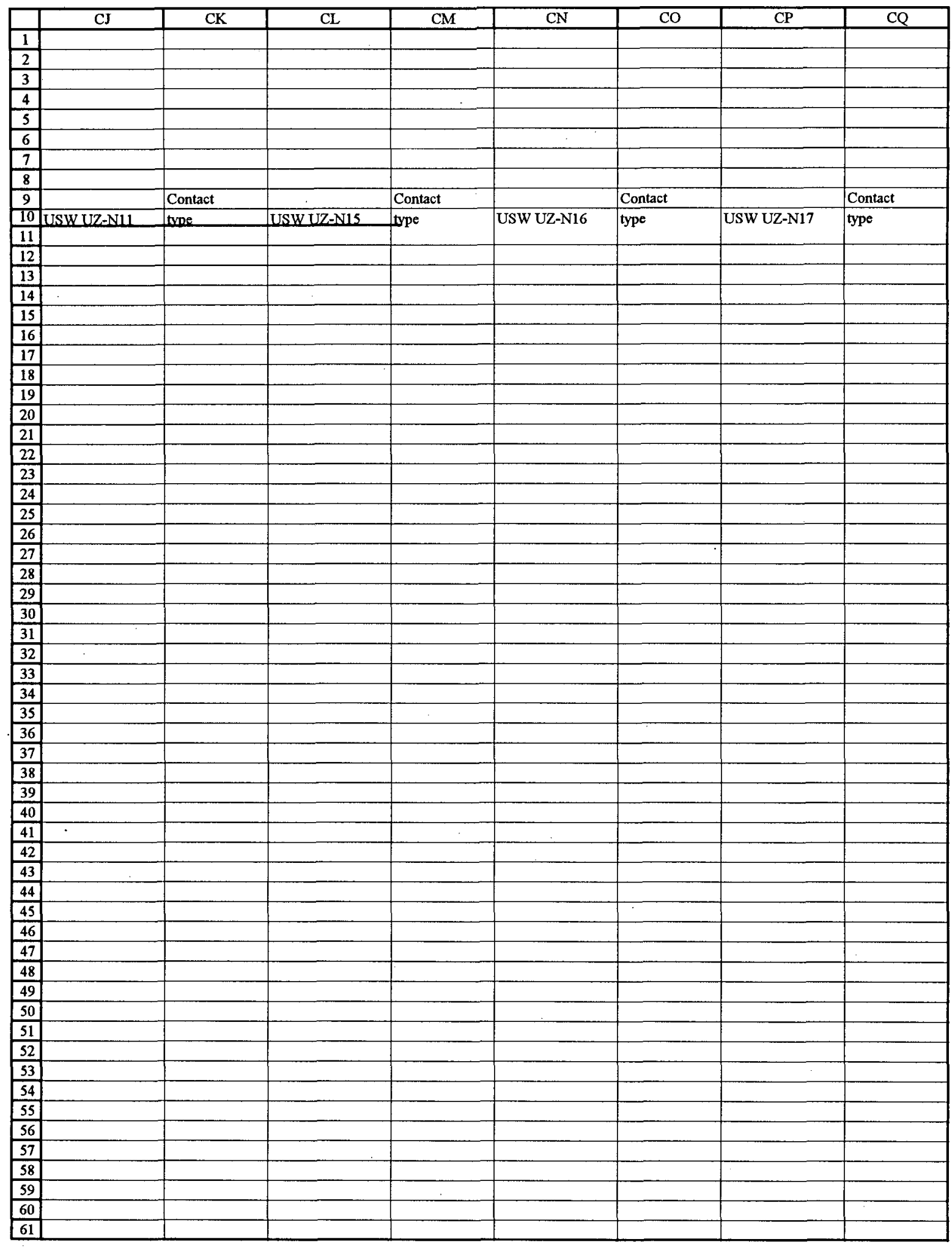



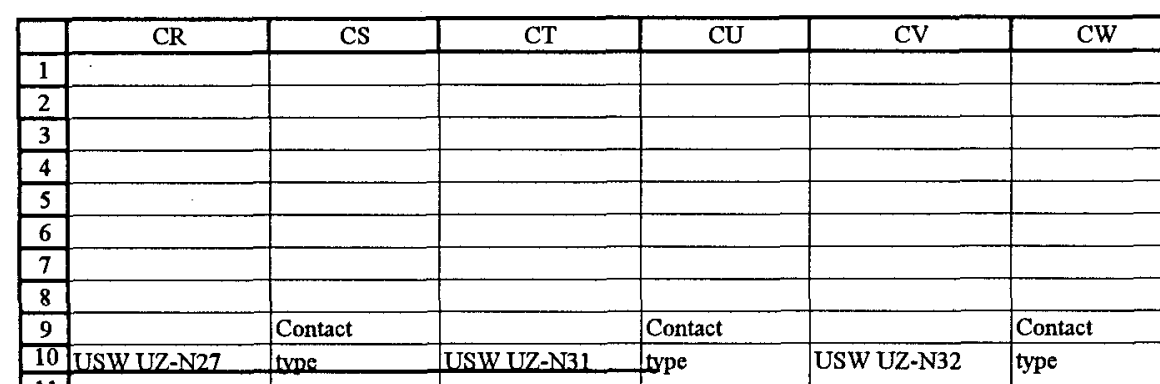

12

\begin{tabular}{ll}
13 \\
\hline 14 \\
\hline
\end{tabular}

15

\begin{tabular}{l}
16 \\
\hline 17 \\
\hline
\end{tabular}

\begin{tabular}{|l|}
\hline 18 \\
\hline 19
\end{tabular}

19

20

$\frac{21}{22}$

23

\begin{tabular}{|l|}
\hline 24 \\
\hline 25 \\
\hline
\end{tabular}

\begin{tabular}{|l|}
\hline 26 \\
\hline 27
\end{tabular}

\begin{tabular}{|l|}
\hline 27 \\
\hline 28
\end{tabular}

\begin{tabular}{|l|}
\hline 28 \\
\hline 29
\end{tabular}

30

31

\begin{tabular}{l|}
\hline 32 \\
\hline 33 \\
\hline 34
\end{tabular}

\begin{tabular}{|l|}
\hline 34 \\
\hline 35
\end{tabular}

35

\begin{tabular}{|l|}
\hline 36 \\
\hline 37 \\
\hline 38
\end{tabular}

\begin{tabular}{l}
38 \\
\hline 39 \\
\hline
\end{tabular}

\begin{tabular}{|l|}
\hline 40 \\
\hline 41
\end{tabular}

\begin{tabular}{ll}
41 \\
\hline 42 \\
\hline
\end{tabular}

43

44

45

\begin{tabular}{|l|}
\hline 46 \\
\hline 47 \\
\hline
\end{tabular}

\begin{tabular}{|l|}
\hline 48 \\
\hline 49 \\
\hline 50
\end{tabular}

\begin{tabular}{|r|}
\hline 48 \\
\hline 50 \\
\hline 59
\end{tabular}

\begin{tabular}{|l|}
\hline 50 \\
\hline 51 \\
\hline 52
\end{tabular}

\begin{tabular}{|l|}
\hline 52 \\
\hline 53 \\
\hline 54 \\
\hline
\end{tabular}

\begin{tabular}{|l|}
\hline 53 \\
\hline 54 \\
\hline 55 \\
\hline
\end{tabular}

\begin{tabular}{|l}
55 \\
\hline 56 \\
\hline 57
\end{tabular}

\begin{tabular}{|l|}
\hline 56 \\
\hline 57 \\
\hline 58 \\
\hline
\end{tabular}

\begin{tabular}{|l|}
\hline 58 \\
\hline 59 \\
\hline 60
\end{tabular}

59

\begin{tabular}{l|}
60 \\
\hline 61 \\
\hline
\end{tabular}

type

USW UZ-N32 type

\begin{tabular}{|l|l|l|}
\hline & $\mathrm{CX}$ & $\mathrm{CY}$ \\
\hline & & \\
& & \\
& & \\
& \\
& & \\
& & \\
\hline & USW UZ-N33 & Contact \\
\hline
\end{tabular}




\begin{tabular}{|c|c|c|c|c|c|c|c|c|}
\hline & $\mathrm{CZ}$ & $\overline{\mathrm{DA}}$ & $\overline{\mathrm{DB}}$ & DC & $\mathrm{DD}$ & $\overline{\mathrm{DE}}$ & DF & $\overline{D G}$ \\
\hline \multirow{2}{*}{\multicolumn{9}{|c|}{$\frac{1}{2}$}} \\
\hline & & & & & & & & \\
\hline \multicolumn{9}{|l|}{3} \\
\hline \multicolumn{9}{|l|}{4} \\
\hline \multicolumn{9}{|l|}{5} \\
\hline \multicolumn{9}{|l|}{6} \\
\hline \multicolumn{9}{|l|}{7} \\
\hline \multicolumn{9}{|l|}{8} \\
\hline 9 & & Contact & & Contact & & Contact & & \\
\hline 10 & USW UZ-N 34 & tyoe & USW UZ-N35 & type & USW UZ-N36 & type & & Symbol \\
\hline \multicolumn{9}{|l|}{11} \\
\hline \multicolumn{9}{|l|}{12} \\
\hline \multicolumn{9}{|l|}{13} \\
\hline \multicolumn{9}{|l|}{14} \\
\hline \multicolumn{9}{|l|}{15} \\
\hline \multicolumn{9}{|l|}{16} \\
\hline \multicolumn{9}{|l|}{17} \\
\hline 18 & & & & & & & & \\
\hline 19 & & & & & & & & \\
\hline 20 & & & & & & & & \\
\hline 21 & & & & & & & & \\
\hline 22 & & & & & & & & \\
\hline 23 & & & & & & & & \\
\hline 24 & & & & & & & & \\
\hline 25 & & & & & & & & \\
\hline 26 & & & & & & & & \\
\hline 27 & & & & & & & & \\
\hline 28 & & & & & & & & \\
\hline 29 & & & & & & & & \\
\hline 30 & & & & & & & & \\
\hline 31 & & & & & & & & \\
\hline 32 & & & & & & & & \\
\hline 33 & & & & & & & & \\
\hline 34 & & & & & & & & \\
\hline 35 & & & & & & & & \\
\hline 36 & & & & & & & & \\
\hline 37 & & & & & & & & \\
\hline 38 & & & & & & & & \\
\hline 39 & & & & & & & & \\
\hline 40 & & & & & & & & \\
\hline 41 & & & & & & & & \\
\hline 42 & & & & & & & & \\
\hline 43 & & & & & & & & \\
\hline 44 & & & & & & & & \\
\hline 45 & & & & & & & & \\
\hline 46 & & & & & & & & \\
\hline 47 & & & & & & & & \\
\hline 48 & & & & & & & & \\
\hline 49 & & & & & & & & \\
\hline 50 & & & & & & & & \\
\hline 51 & & & & & & & & \\
\hline 52 & & & & & & & & \\
\hline 53 & & & & & & & & \\
\hline 54 & & & & & & & & \\
\hline 55 & & & & & & & & \\
\hline 56 & & & & & & & & \\
\hline 57 & & & & & & & & \\
\hline 58 & & & & & & & & \\
\hline 59 & & & & & & & & \\
\hline 60 & & & & & & & & \\
\hline 61 & & & & & & & & \\
\hline
\end{tabular}


TDR-NBS-GS-000007

Attachment VII

p. VII-15 of 22

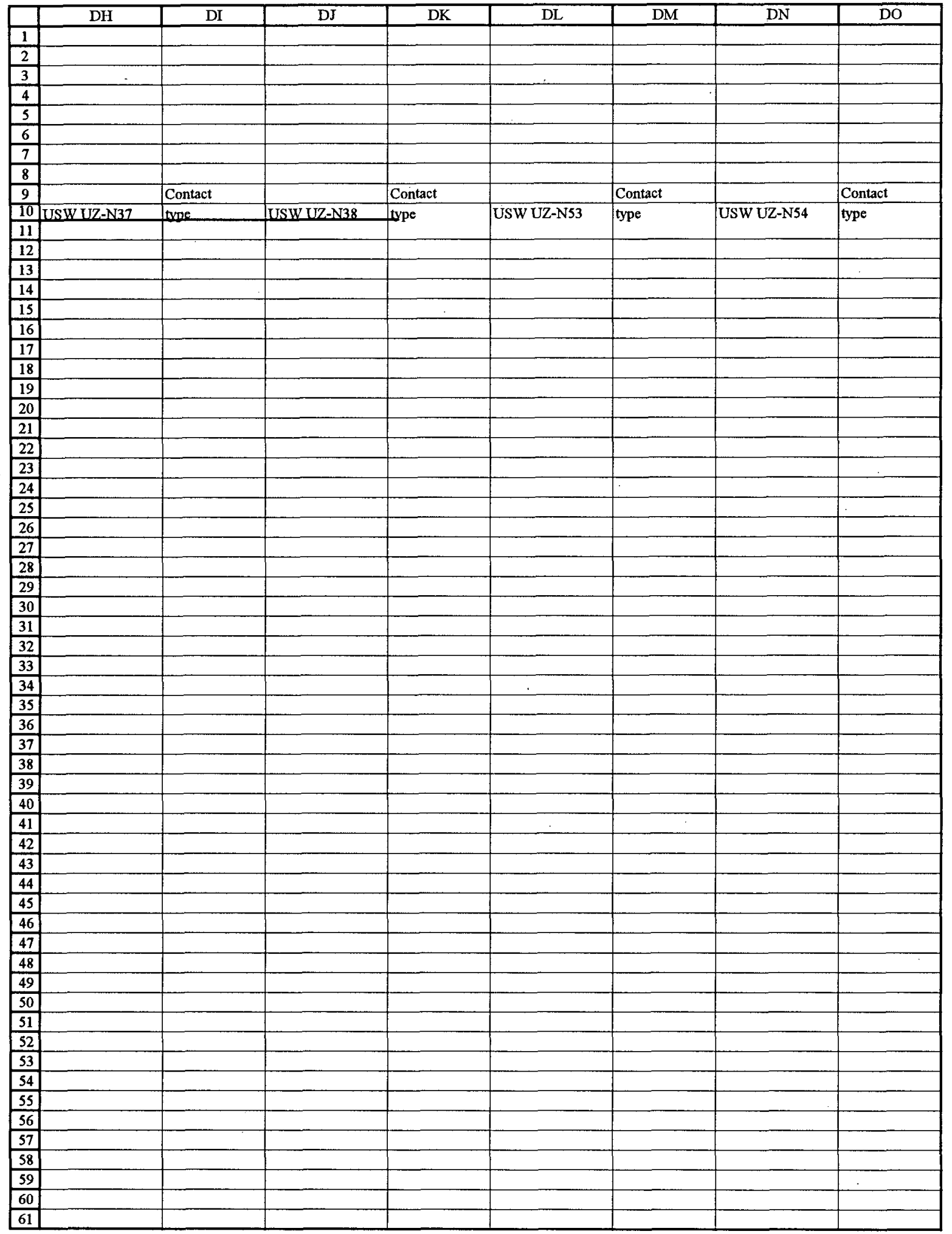


TDR-NBS-GS-000007

Attachment VII

p. VII-16 of 22

\begin{tabular}{|c|c|c|c|c|c|c|c|c|}
\hline & $\overline{\mathrm{DP}}$ & $\overline{\mathrm{DQ}}$ & $\overline{\mathrm{DR}}$ & DS & $\overline{\mathrm{DT}}$ & $\mathrm{DU}$ & DV & $\overline{\mathrm{DW}}$ \\
\hline \multicolumn{9}{|l|}{1} \\
\hline \multirow{2}{*}{\multicolumn{9}{|c|}{$\frac{2}{3}$}} \\
\hline & & & & & & & & \\
\hline \multirow{2}{*}{\multicolumn{9}{|c|}{4}} \\
\hline \multirow{2}{*}{\multicolumn{6}{|c|}{5}} & & & \\
\hline 6 & & & & & & & & \\
\hline \multicolumn{9}{|l|}{7} \\
\hline \multicolumn{9}{|l|}{8} \\
\hline \begin{tabular}{l|l}
9 \\
\end{tabular} & & Contact & & Contact & & Contact & & Contact \\
\hline 10 & USW UZ-N5S & tve & \multicolumn{6}{|c|}{$\frac{10}{11}$ USW UZ-NSS } \\
\hline \begin{tabular}{l|}
11 \\
12 \\
\end{tabular} & & & \\
\hline 13 & & & & & & & & \\
\hline \multicolumn{9}{|l|}{14} \\
\hline \multicolumn{9}{|l|}{15} \\
\hline \multirow{2}{*}{\multicolumn{9}{|c|}{$\frac{16}{17}$}} \\
\hline & & & & & & & & \\
\hline \multicolumn{9}{|l|}{18} \\
\hline \multicolumn{9}{|l|}{19} \\
\hline \multirow{2}{*}{\multicolumn{9}{|c|}{$20 \mid$}} \\
\hline & & & & & & & & \\
\hline 22 & & & & & & & & \\
\hline 23 & & & & & & & & \\
\hline 24 & & & & & & & & \\
\hline 25 & & & & & & & & \\
\hline 26 & & & & & & & & \\
\hline 27 & & & & & & & & \\
\hline 28 & & & & & & & & \\
\hline 29 & & & & & & & & \\
\hline 30 & & & & & & & & \\
\hline 31 & & & & & & & & \\
\hline 32 & & & & & & & & \\
\hline 33 & & & & & & & & \\
\hline 34 & & & & & & & & \\
\hline 35 & & & & & & & & \\
\hline 36 & & & & & & & & \\
\hline 37 & & & & & & & & \\
\hline 38 & & & & & & & & \\
\hline \begin{tabular}{|l|}
39 \\
\end{tabular} & & & & & & & & \\
\hline \begin{tabular}{|l|}
400 \\
\end{tabular} & & & & & & & & \\
\hline 41 & & & & & & & & \\
\hline 42 & & & & & & & & \\
\hline 43 & & & & & & & & \\
\hline 44 & & & & & & & & \\
\hline 45 & & & & & & & & \\
\hline 46 & & & & & & & & \\
\hline 47 & & & & & & & & \\
\hline 48 & & & & & & & & \\
\hline 49 & & & & & & & & \\
\hline 50 & & & & & & & & \\
\hline 51 & & & & & & & & \\
\hline 52 & & & & & & & & \\
\hline 53 & & & & & & & & \\
\hline 54 & & & & & & & & \\
\hline 55 & & & & & & & & \\
\hline 56 & & & & & & & & \\
\hline 57 & & & & & & & & \\
\hline 58 & & & & & & & & \\
\hline 59 & & & & & & & & \\
\hline 60 & & & & & & & & \\
\hline 61 & & & & & & & & \\
\hline
\end{tabular}




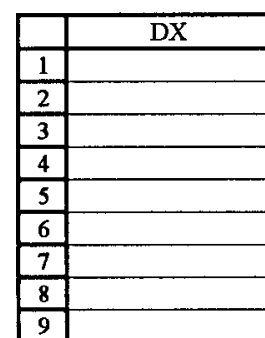

10 USW UZ-N61

11

12

14

15

16

\begin{tabular}{l}
17 \\
\hline 18
\end{tabular}

19

20

21

23

24

\begin{tabular}{|l|}
\hline 25 \\
\hline 26 \\
\hline
\end{tabular}

\begin{tabular}{l}
26 \\
27 \\
\hline 28
\end{tabular}

\begin{tabular}{|l|}
\hline 28 \\
\hline 29 \\
\hline 30
\end{tabular}

\begin{tabular}{|l|}
\hline 29 \\
\hline 30
\end{tabular}

31

32

33

\begin{tabular}{|l|}
34 \\
\hline 35 \\
\hline 36
\end{tabular}

36

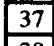

\begin{tabular}{l}
38 \\
\hline 39 \\
\hline 40
\end{tabular}

40

\begin{tabular}{|l|}
\hline 41 \\
\hline 42 \\
\hline
\end{tabular}

42

\begin{tabular}{|l|}
\hline 43 \\
\hline 44 \\
\hline
\end{tabular}

\begin{tabular}{|l|}
\hline 44 \\
\hline 45 \\
\hline 46 \\
\hline
\end{tabular}

46

\begin{tabular}{|l|}
\hline 47 \\
\hline 48
\end{tabular}

48

\begin{tabular}{|l|}
\hline 49 \\
\hline 50
\end{tabular}

\begin{tabular}{|l|}
51 \\
\hline 52 \\
\hline
\end{tabular}

53

54

\begin{tabular}{|l|}
\hline 55 \\
\hline 56 \\
\hline
\end{tabular}

\begin{tabular}{|l|}
\hline 57 \\
\hline 58 \\
\hline
\end{tabular}

59

60

\begin{tabular}{|l|}
\hline 61 \\
\hline
\end{tabular}

Con

Contact

tupe

USW UZ-N62

type

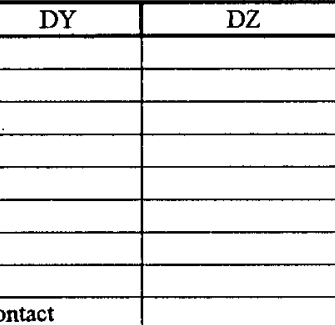

EB
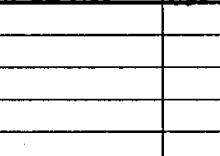

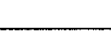




\begin{tabular}{|c|c|c|c|c|c|c|c|c|c|}
\hline & $\overline{E F}$ & $\overline{\mathrm{EG}}$ & EH & $\overline{\text { EI }}$ & EJ & EK & EL & $\mathrm{EM}$ & $\mathrm{EN}$ \\
\hline 1 & & & & & & & & & \\
\hline 2 & & & & & & & & & \\
\hline 3 & & & & & & & & & \\
\hline 4 & & & & & & & & & \\
\hline 5 & & & & & & & & & \\
\hline 6 & & & & & & & & & \\
\hline 7 & & & & & & & & & \\
\hline 8 & & & & & & & & & \\
\hline 9 & & Contact & & Contact & & Contact & & Contact & \\
\hline 10 & USW WT-1 & twoe & USW WT-2 & type & UE-25 WT\#3 & type & UE-25 WT\#4 & type & UE-25 WT\#6 \\
\hline 11 & 0.0 & & 0.0 & & 0.0 & & 0.0 & & 0.0 \\
\hline 12 & 0.0 & & 0.0 & & 0.0 & & 0.0 & & 0.0 \\
\hline 13 & 30.0 & & 60.0 & & 11.0 & & 51.0 & & 170.0 \\
\hline 14 & 30.0 & & 60.0 & & 11.0 & & 51.0 & & 170.0 \\
\hline 15 & 30.0 & & 60.0 & & 11.0 & & 51.0 & & 170.0 \\
\hline 16 & 395.0 & & 193.0 & & 11.0 & & 261.0 & & 170.0 \\
\hline 17 & 410.0 & & 200.0 & & 11.0 & & 261.0 & & 170.0 \\
\hline 18 & 417.0 & & 215.0 & & 11.0 & & 270.0 & & 170.0 \\
\hline 19 & 431.0 & & 227.0 & & 11.0 & & 281.0 & & 170.0 \\
\hline 20 & 435.0 & & 230.0 & & 11.0 & & 293.0 & & 170.0 \\
\hline \begin{tabular}{l|l}
21 \\
\end{tabular} & 435.0 & & 230.0 & & 11.0 & & 293.0 & & 170.0 \\
\hline 22 & 446.0 & & 247.0 & & 11.0 & & 324.0 & & 170.0 \\
\hline 23 & 446.0 & & 247.0 & & 11.0 & & 419.0 & & 170.0 \\
\hline 24 & 477.0 & & 271.0 & & 11.0 & & 444.0 & & 170.0 \\
\hline 25 & 481.0 & & 275.0 & & 11.0 & & 448.0 & & 170.0 \\
\hline 26 & 484.0 & & 280.0 & & 11.0 & & 456.0 & & 170.0 \\
\hline 27 & 492.0 & & 285.0 & & 11.0 & & 458.0 & & 170.0 \\
\hline 28 & 575.0 & & 380.0 & & 11.0 & & 630.0 & & 170.0 \\
\hline 29 & 593.0 & & 421.0 & & 11.0 & & 660.0 & & 170.0 \\
\hline 30 & 593.0 & & 421.0 & & 11.0 & & 660.0 & & 250.0 \\
\hline 31 & 733.0 & & 590.0 & & 11.0 & & 727.0 & & 250.0 \\
\hline 32 & 888.0 & & 727.0 & & 11.0 & & 785.0 & & 250.0 \\
\hline 33 & 1187.0 & & 1014.0 & & 35.0 & & 1091.0 & & 303.0 \\
\hline 34 & 1299.0 & & 1179.0 & & 189.0 & & 1091.0 & & 303.0 \\
\hline 35 & 1337.0 & & 1223.0 & & 293.0 & & 1122.0 & & 337.0 \\
\hline 36 & 1368.0 & & 1264.0 & & 327.0 & & 1141.0 & & 352.0 \\
\hline 37 & 1380.0 & & 1315.0 & & 351.0 & & 1150.0 & & 369.0 \\
\hline 38 & 1384.0 & & 1319.0 & & 358.0 & & 1156.0 & & 383.0 \\
\hline 39 & 1564.0 & & 1521.0 & & 461.0 & & & & \\
\hline 40 & 1564.0 & & 1594.0 & & 512.0 & & & & \\
\hline 41 & 1564.0 & & 1594.0 & & 554.0 & & & & \\
\hline 42 & 1564.0 & & 1706.0 & & 660.0 & & & & \\
\hline 43 & 1564.0 & & 1776.0 & & 704.0 & & & & \\
\hline 44 & 1564.0 & & 1794.0 & & 710.0 & & & & \\
\hline 45 & 1564.0 & & & & 835.0 & & & & \\
\hline 46 & 1564.0 & & . & & 846.0 & & & & \\
\hline 47 & 1564.0 & & & & 850.0 & & & & \\
\hline 48 & 1564.0 & & & & 909.0 & & & & \\
\hline \begin{tabular}{l|l|}
49 \\
\end{tabular} & & & & & & & & & \\
\hline 50 & & & & & & & & & \\
\hline 51 & & & & & & & & & \\
\hline 52 & & & & & & & & & \\
\hline 53 & & & & & & & & & \\
\hline 54 & & & & & & & & & \\
\hline 55 & & & & & & & & & \\
\hline 56 & & & & & & & & & \\
\hline 57 & & & & & & & & & \\
\hline 58 & & & & & & & & & \\
\hline 59 & & & & & & & & & \\
\hline 60 & 1337.0 & & 1452.0 & & 293.0 & & 1122.0 & & 305.0 \\
\hline 61 & 713.0 & & 540.0 & & & & 679.0 & & \\
\hline
\end{tabular}




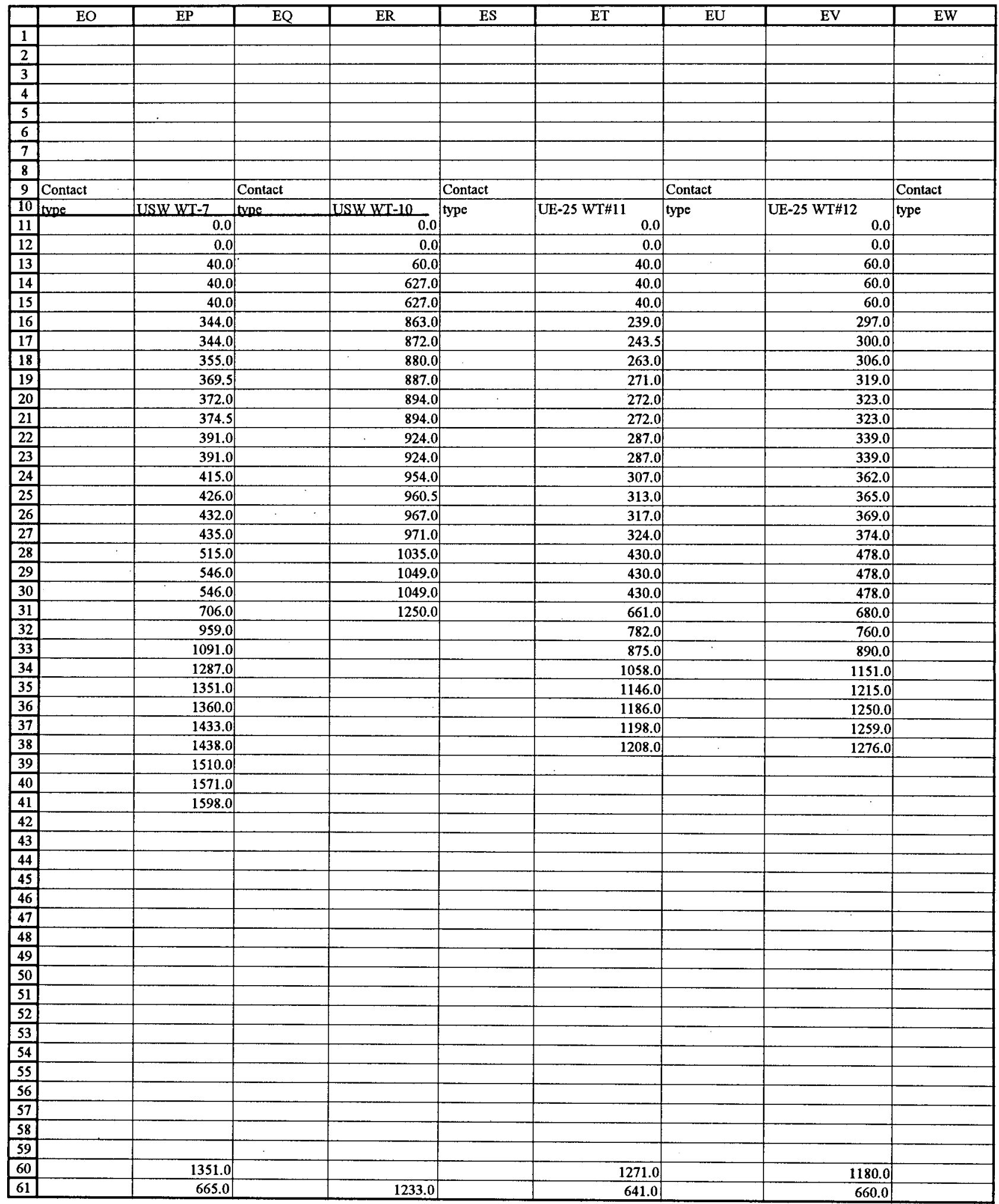




\begin{tabular}{|c|c|c|c|c|c|c|c|c|}
\hline & EX & EY & $\mathrm{EZ}$ & $\mathrm{FA}$ & FB & $\mathrm{FC}$ & $\mathrm{FD}$ & $\overline{F E}$ \\
\hline \multicolumn{9}{|l|}{1} \\
\hline 2 & & & & & & & & \\
\hline \multicolumn{9}{|l|}{3} \\
\hline \multicolumn{9}{|l|}{4} \\
\hline \multicolumn{9}{|l|}{5} \\
\hline \multicolumn{9}{|l|}{6} \\
\hline \multicolumn{9}{|l|}{7} \\
\hline \multicolumn{9}{|l|}{8} \\
\hline 9 & & Contact & & Contact & & Contact & & Contact \\
\hline 10 & UJE-25 WT\#13 & type & UE-25WT\#14 & type & UE-25 WT\#15 & type & UE-25 WT\#16 & type \\
\hline 11 & 0.0 & & 0.0 & & 0.0 & & 0.0 & \\
\hline 12 & 0.0 & & 0.0 & & 0.0 & & 0.0 & \\
\hline 13 & 220.0 & & 107.0 & & 210.0 & & 137.0 & \\
\hline 14 & 220.0 & & 107.0 & & 210.0 & & 137.0 & \\
\hline 15 & 220.0 & & 107.0 & & 210.0 & & 137.0 & \\
\hline 16 & 416.0 & & 107.0 & & 332.0 & & 368.0 & \\
\hline 17 & 416.0 & & 107.0 & & 332.0 & & 368.0 & \\
\hline 18 & 427.0 & & 107.0 & & 334.0 & & 375.0 & \\
\hline 19 & 440.0 & & 107.0 & & 349.0 & & 386.0 & \\
\hline 20 & 450.0 & & 107.0 & & 356.0 & & 395.0 & \\
\hline 21 & 450.0 & & 107.0 & & 356.0 & & 395.0 & \\
\hline 22 & 460.0 & & 107.0 & & 372.0 & & 462.0 & \\
\hline 23 & 469.0 & & 107.0 & & 413.0 & & 558.0 & \\
\hline 24 & 490.0 & & 107.0 & & 436.0 & & 580.0 & \\
\hline 25 & 497.0 & & 122.0 & & 440.0 & & 588.0 & \\
\hline 26 & 498.0 & & 124.0 & & 442.0 & & 594.0 & \\
\hline 27 & 500.0 & & 128.0 & & 444.0 & & 596.0 & \\
\hline 28 & 612.0 & & 247.0 & & 608.0 & & 818.0 & \\
\hline 29 & 630.0 & & 275.0 & & 641.0 & & 830.0 & \\
\hline 30 & 630.0 & & 275.0 & & 641.0 & & 830.0 & \\
\hline 31 & 755.0 & & 446.0 & & 852.0 & & 830.0 & \\
\hline 32 & 868.0 & & 534.0 & & 919.0 & & 830.0 & \\
\hline 33 & 1103.0 & & 830.0 & & 1260.0 & & 1013.0 & \\
\hline 34 & & & 1024.0 & & & & 1013.0 & \\
\hline 35 & & & 1117.0 & & & & 1050.0 & \\
\hline 36 & & & 1137.0 & & & & 1057.0 & \\
\hline 37 & & & 1157.0 & & & & 1068.0 & \\
\hline 38 & & & 1210.0 & & & & 1068.0 & \\
\hline \multicolumn{9}{|l|}{39} \\
\hline 40 & & & & & & & & \\
\hline \multicolumn{9}{|l|}{41} \\
\hline \multicolumn{9}{|l|}{42} \\
\hline \multicolumn{9}{|l|}{43} \\
\hline \multicolumn{9}{|l|}{44} \\
\hline \multicolumn{9}{|l|}{45} \\
\hline \multicolumn{9}{|l|}{46} \\
\hline 47 & & & & & & & & \\
\hline 48 & & & & & & & & \\
\hline 49 & & & & & & & & \\
\hline 50 & & & & & . & & & \\
\hline 51 & & & & & & & & \\
\hline 52 & & & & & & & & \\
\hline 53 & & & & & & & & \\
\hline 54 & & & & & & & & \\
\hline 55 & & & & & & & & \\
\hline 56 & & & & & & & & \\
\hline 57 & & & & & & & & \\
\hline 58 & & & & & & & & \\
\hline 59 & & & & & & & & \\
\hline 60 & & & 1117.0 & & & & 1050.0 & \\
\hline 61 & 740.0 & & 392.0 & & 840.0 & & & \\
\hline
\end{tabular}




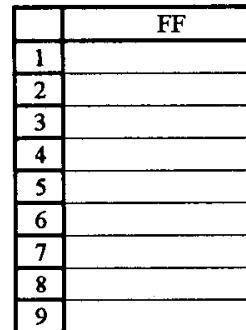

\begin{tabular}{|c|c|c}
\hline & FG & FH \\
\hline & & \\
\hline & & \\
\hline & & \\
\hline & & \\
\hline & & \\
\hline
\end{tabular}

10 UE-25 WTH17

11

12

13

15

16

17

\begin{tabular}{l}
18 \\
\hline 19
\end{tabular}

20

\begin{tabular}{|l|}
\hline 21 \\
\hline 22
\end{tabular}

23

24

26

28

29

30

\begin{tabular}{|l|}
\hline 31 \\
\hline 32 \\
\hline 33
\end{tabular}

33

34

\begin{tabular}{|l|}
\hline 35 \\
\hline 36 \\
\hline
\end{tabular}

37

38

39

40

41

42

43

44

45

46

47

48

49

50

51

53

54

55

\begin{tabular}{l}
56 \\
\hline 57
\end{tabular}

58

\begin{tabular}{|l|}
58 \\
\hline 59 \\
\hline 60
\end{tabular}

60

type

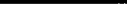

\begin{tabular}{r|r|r}
0.0 & & \\
\hline 30.0 & & \\
\hline 30.0 & & \\
\hline 30.0 & & \\
\hline
\end{tabular}

Contact

0

-

\begin{tabular}{|r|r|}
\hline 188.0 & 0.0 \\
\hline 188.0 & 314.0 \\
\hline 194.0 & 314.0 \\
\hline
\end{tabular}

\begin{tabular}{r|r}
194.0 & 314.0 \\
\hline 197.0 & 332.0 \\
\hline
\end{tabular}

\begin{tabular}{r|r}
197.0 & 340.0 \\
\hline 203.0 & 353.0
\end{tabular}

\begin{tabular}{r|r}
203.0 & 423.0 \\
\hline 217.0 & 497.0
\end{tabular}

\begin{tabular}{r|r}
217.0 & 497.0 \\
\hline 217.0 & 651.0 \\
\hline
\end{tabular}

\begin{tabular}{r|r}
242.0 & 692.0 \\
\hline 245.0 & 698.0
\end{tabular}

\begin{tabular}{r|r}
248.0 & \\
\hline 251.0 & 701.0
\end{tabular}

\begin{tabular}{r|r}
251.0 & 702.0 \\
\hline
\end{tabular}

\begin{tabular}{r|r}
312.0 & 879.0 \\
\hline 336.0 & 900.0
\end{tabular}

\begin{tabular}{r|r}
336.0 & 900.0 \\
\hline 336.0 & 900.0
\end{tabular}

\begin{tabular}{r|r}
472.0 & \\
\hline 535.0 & 1078.0 \\
\hline
\end{tabular}

\begin{tabular}{r|r}
535.0 & 1170.0 \\
6668.0 & 1501.0
\end{tabular}

\begin{tabular}{r|r}
\hline 874.0 & 1501.0 \\
\hline 959.0 & 1501.0
\end{tabular}

\begin{tabular}{r|r}
874.0 & 1501.0 \\
\hline 959.0 & 1564.0 \\
\hline
\end{tabular}

\begin{tabular}{r|r}
989.0 & 1592.0
\end{tabular}

\begin{tabular}{r|r}
998.0 & 1620.0 \\
\hline 998.0 & 1620.0
\end{tabular}

\begin{tabular}{r|r}
998.0 & 1620.0 \\
\hline 998.0 & 1620.0
\end{tabular}

998.0
1184.0

1271.0

1313.0

1318.0

\begin{tabular}{|l|}
\hline FI \\
\hline \\
\hline \\
\hline \\
\hline \\
\hline \\
\hline \\
\hline \\
\hline
\end{tabular}

.0

$+2$

$+$

-

0

1.0

900.0

78.0

1.0

20.0

\begin{tabular}{|l|l|}
\hline \\
\hline
\end{tabular}

\begin{tabular}{l|l|l|l}
1313.0 & & \\
\hline & & & \\
\hline
\end{tabular}

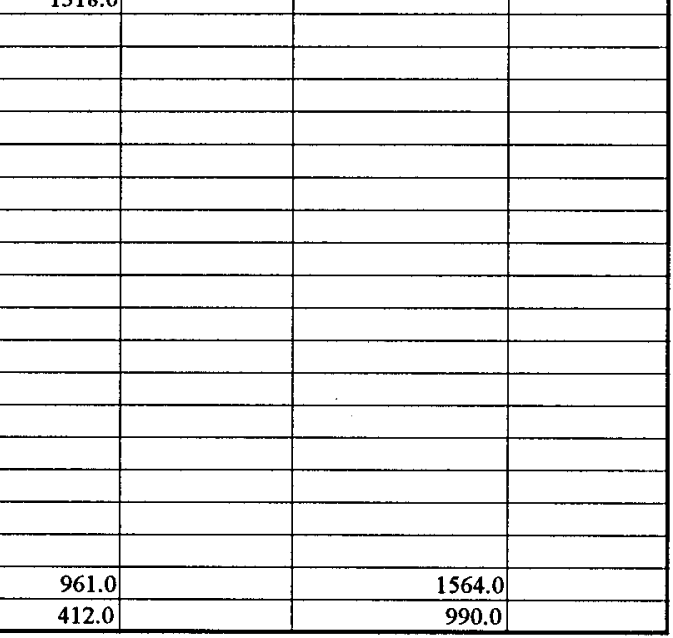




\begin{tabular}{|c|c|}
\hline & A \\
\hline 62 & \\
\hline 63 & Symbols: \\
\hline 64 & Contact type: $d, w, v, c, a(a c, a z), n p, n p-e(p e), n p-f(t, i, b), n p-t d, n f, n e, n i, g, n d$ \\
\hline 65 & $\mathrm{~d}$ - deposition; $\mathrm{w}$ - welding; $\mathrm{v}$ - vitric; $\mathrm{c}$ - crystallization; $\mathrm{a}$ - alteration ( $\mathrm{ac}$ - clay; az - \\
\hline 66 & np - not penetrated; np-e - eroded (pe - partially eroded); np-f - faulted \\
\hline 67 & $\mathrm{ft}$ - faulted top, $\mathrm{fi}$ - faulted interior, $\mathrm{fb}$ - faulted base \\
\hline 68 & np-td - total depth in s \\
\hline 69 & g・ geophysical lo \\
\hline 70 & ly altered to zeolite (and/or clay) are indic \\
\hline 71 & $\begin{array}{l}\text { faz - rocks from the crystal-poor, vitric, densely welded subzone of the Topopah Spring TV } \\
\text { that have significant alteration along (typically high-angle) fractures }\end{array}$ \\
\hline & $\begin{array}{l}\text { Contacts between vitric, crystallized, and zeolitic material are hyphenated to emphasize } \\
\text { fundamental boundaries. }\end{array}$ \\
\hline 73 & \\
\hline 74 & Foot \\
\hline 75 & $1 \mathrm{In} \mathrm{CH}$ \\
\hline & $\begin{array}{l}2 \text { In C\#1, 2, and } 3 \text {-- The lowest unit (Tct2c) locally contains brecciated intervals: C\#1 } \\
\text { (2775 to } 2975 \mathrm{ft} \text { ), C\#2 (2775 to 2935), and C\#3 (2800 to 3000[total depth]). }\end{array}$ \\
\hline 77 & 3 In C\#3 - The lo \\
\hline 78 & $\begin{array}{l}4 \text { In G-1 - Tcpm is not well developed. The density log indicates the rocks are in the upper } \\
\text { part of the partially welded subzone to lower part of the moderately welded subzone and } \\
\text { crystallized. The Tcpm unit could be modeled as absent in this location if unit density and } \\
\text { calculated porosity are the dominant modeling parameters. }\end{array}$ \\
\hline 79 & $\begin{array}{l}5 \text { In G-1 - The total thickness of Tct approximately the same as } \mathrm{H}-1 . \mathrm{Tc} 2 \mathrm{v} \text { is thicker in G- } \\
1 \text { than } \mathrm{H}-1, \text { but } \mathrm{Tctm} \text { is correspondingly thinner. The character of the upper part of } \mathrm{Tctm} \\
\text { and possibly lower part of Tct } 2 \mathrm{c} \text { appear to differ from the typical profile. }\end{array}$ \\
\hline 80 & $6 \mathrm{In} G$ \\
\hline 3 & $\begin{array}{l}7 \text { In G-2 - The Tram Tuff in th } \\
\text { percent to } 40 \text { percent) and the en }\end{array}$ \\
\hline 81 & Occurre \\
\hline 82 & $\begin{array}{l}8 \text { In G-4 - Tcpm is not well developed. The density log indicates the rocks are in the upper } \\
\text { part of the partially welded subzone to lower part of the moderately welded subzone and } \\
\text { crystallized. The Tcpm unit could be modeled as absent in this location if unit density and } \\
\text { calculated porosity are the dominant modeling parameters. }\end{array}$ \\
\hline 83. & $\begin{array}{l}9 \text { In H-1 - Tcpm is not well developed. The density log indicates the rocks are in the upper } \\
\text { part of the partially welded subzone to lower part of the moderately welded subzone and } \\
\text { crystallized. The Tcpm unit could be modeled as absent in this location if unit density and } \\
\text { calculated porosity are the dominant modeling parameters. }\end{array}$ \\
\hline 84 & $\begin{array}{l}10 \text { In H-6 - Tcpm is not well developed. The density log indicates the rocks are in the } \\
\text { upper part of the partially welded subzone to lower part of the moderately welded subzone } \\
\text { and crystallized. The Tcpm unit could be modeled as absent in this location if unit density } \\
\text { and calculated porosity are the dominant modeling parameters. }\end{array}$ \\
\hline 85 & $\begin{array}{l}11 \text { In UZ-14 - Tcpm is not well developed. The density log indicates the rocks are in the } \\
\text { upper part of the partially welded subzone to lower part of the moderately welded subzone } \\
\text { and crystallized. The Tcpm unit could be modeled as absent in this location if unit density } \\
\text { and calculated porosity are the dominant modeling parameters. }\end{array}$ \\
\hline & ult is inferred \\
\hline
\end{tabular}




\section{Attachment VIII: \\ Special Case Data Recommended to be Qualified}

3-page spreadsheet numbered separately 


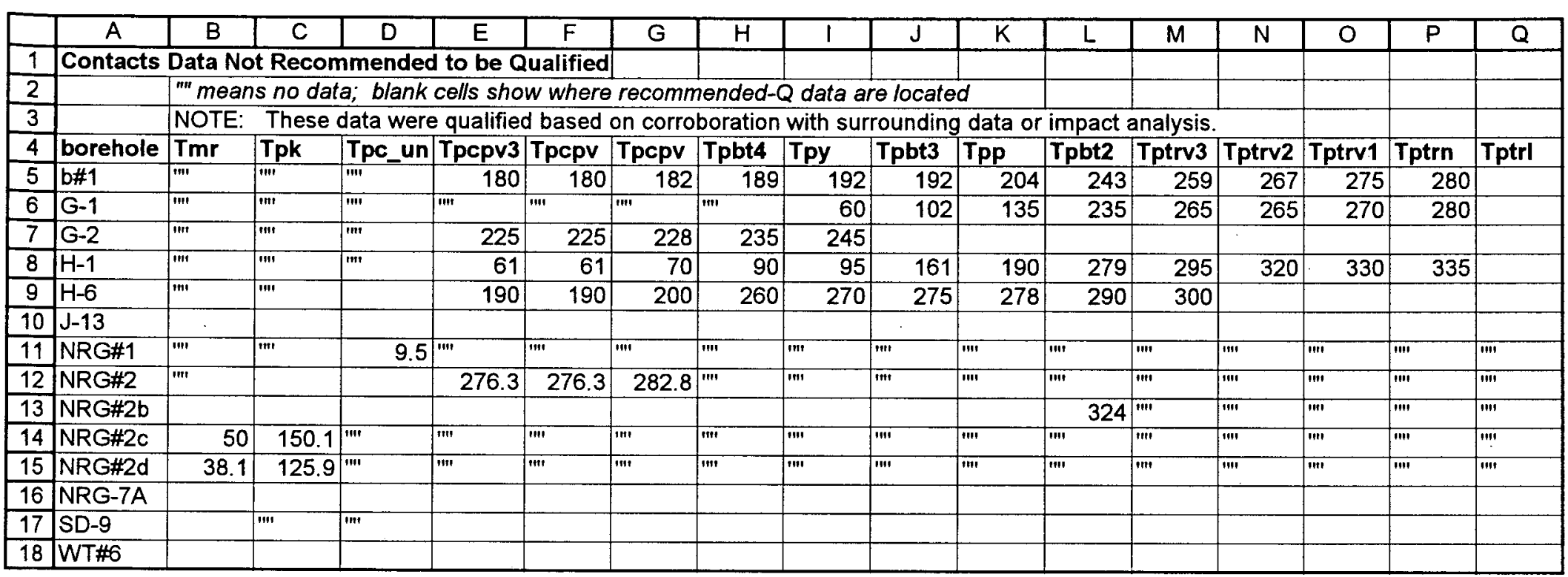




\begin{tabular}{|c|c|c|c|c|c|c|c|c|c|c|c|c|c|c|c|c|c|c|}
\hline & $\mathrm{R}$ & $S$ & $T$ & $U$ & $\mathrm{~V}$ & $\bar{W}$ & $x$ & $\bar{Y}$ & $\bar{Z}$ & $\overline{\mathrm{AA}}$ & $A B$ & $A C$ & $A D$ & $\overline{\mathrm{AE}}$ & AF & $\overline{A G}$ & $\overline{\mathrm{AH}}$ & $\overline{\mathrm{Al}}$ \\
\hline \multicolumn{19}{|l|}{1} \\
\hline 2 & & & & & & & & & & & & . & & & & & & \\
\hline \multicolumn{19}{|l|}{3} \\
\hline 4 & Tptf & Tptpul & RHH & Tptpmn & Tptpll & Tptpin & Tptpv3 & Tptpv2 & Tptpv1 & Tpbt1 & Tac & Tacbt & Tcpuv & Tcpuc & Tcpm & Tcplc & Tcplv & Tcpbt \\
\hline \multicolumn{19}{|l|}{5} \\
\hline \multirow{2}{*}{\multicolumn{17}{|c|}{$\frac{0}{7}$}} & & \\
\hline & & & & & & & & & & & & & & & & & & \\
\hline \multicolumn{19}{|l|}{8} \\
\hline \multicolumn{19}{|l|}{9} \\
\hline 10 & & & & & & & & & & & & 1682 & 1711 & 1742 & 1848 & 1942 & 1961 & 1993 \\
\hline 11 & "'" & it"' & '"' & "'"' & '"' & "'"' & "'"' & "'"' & "'+' & "'"' & '"' & '"' & \begin{tabular}{|l|}
$" ' \prime$ \\
\end{tabular} & "'"' & "'"' & I'"' & '"' & '"'! \\
\hline 12 & "'"' & '"' & "'"' & "'"' & '"' & "'!' & "'"' & "'"' & '"'! & '"' & '"' & "'" & "'" & "'"' & "'"' & "'"' & "'i' & "'"' \\
\hline 13 & '"' & "'"' & "'!' & "'t' & "'"' & "'"' & I"' & 'm! & '"'! & "'! & "'"' & "'"' & "'" & '"'! & '"' & "'"' & $\mid$ & $\mid$ \\
\hline 14 & "'"' & "'"' & "'"' & "'" & '"' & "'"' & "itt & 'm" & '"'!' & "'i' & '"' & $\mid$ & "'"' & \begin{tabular}{|l|}
$11, '$ \\
\end{tabular} & \begin{tabular}{|l|l} 
\\
\end{tabular} & $\mid$ & "'"' & $\mid$ \\
\hline 15 & '"' & '"' & '"' & "'" & '"' & '"' & "'"' & '"'" & '"' & "'"' & '"'t & "'"' & "'" & "'"' & '"' & "'!' & '"' & "'t' \\
\hline 16 & & & & & & & 1414.8 & 1457 & 1474.6 & 1493 & 1498 & "'"' & "'"' & $\mid$ & "'"' & '"' & '"'!' & $\mid$ \\
\hline 17 & & & & & & & & & & & 1464.1 & 1479.9 & 1764.4 & 1820.7 & 1868.7 & 1938.5 & 1991.4 & 2015.8 \\
\hline 18 & & & & 250 & 250 & & & & & & & '"' & "'"' & '"' & '"'! & 'i'" & '"' & '"' \\
\hline
\end{tabular}




\begin{tabular}{|c|c|c|c|c|c|c|c|c|c|c|c|c|c|}
\hline & $\overline{A J}$ & $\overline{A K}$ & $\overline{A L}$ & $A M$ & $\overline{A N}$ & $\mathrm{AO}$ & AP & $\overline{A Q}$ & $\overline{A R}$ & $\overline{A S}$ & $\overline{A T}$ & $\overline{A U}$ & $\mathrm{AV}$ \\
\hline 1 & & & & & & & & & & & & & \\
\hline 2 & & & & & & & & & & & & & \\
\hline 3 & & & & & & & & & & & & & \\
\hline 4 & Tcbuv & Tcbuc & Tcbm & Tcblc & Tcblv & Tcbbt & Tctuv & Tctuc & Tctm & Tctlc & Tctlv & Tetbt & Tund \\
\hline 5 & & & & & & & & & & & & & \\
\hline 6 & & & & & & & & & & & & & \\
\hline 7 & & & & & & & & & & & & & \\
\hline 8 & & & & & & & & & & & & & \\
\hline 9 & & & . & & & & & & & & & & \\
\hline 10 & 2017 & 2017 & 2082 & 2322 & 2322 & 2322 & 2358 & 2465 & 2658 & 2862 & 2991 & 3200 & 3220 \\
\hline 11 & "'"' & "'"' & '"' & "'! & "'! & '"' & "'"' & "'" & "'!' & "'"' & "'"' & "'"' & "'"' \\
\hline 12 & "'"' & "'i' & '"' & "'"' & "'"' & "'"' & 'i"' & '"' & '"' & "'"' & "'"' & "'"' & "'"' \\
\hline 13 & '"' & '"'", & "'"' & "'"' & "'"' & "i"' & "'+' & "'"' & "i'! & "'"' & "'"' & "'"' & "'"' \\
\hline 14 & "'i" & "'"' & '"' & "'"' & '"'! & "i!' & imi & "'"' & '"'! & "'"' & "'"' & "'"' & "'"' \\
\hline 15 & "'"' & '"'! & '"' & "'"' & '"'! & '"' & I'i' & "'"' & '"'! & "'"' & "'"' & "'"' & "'"' \\
\hline 16 & '"'" & "'" & '"' & "'"' & '"' & "'" & "'"' & "'"' & '"' & "'" & '"'" & "'"' & I"'t \\
\hline 17 & "i"' & '"'" & "'"' & "'"' & "'"' & "'"' & "'! & "'"' & "'"' & "'" & '"'! & '"'! & "'"' \\
\hline 18 & "'"' & "'"' & '"'t' & "'"' & "'"' & '"'"' & '"'! & "'"' & "'"' & "'" & '"'t' & in' & "'"' \\
\hline
\end{tabular}

